\title{
Birational geometry of cluster algebras
}

\author{
Mark Gross, Paul Hacking and Sean Keel
}

\begin{abstract}
We give a geometric interpretation of cluster varieties in terms of blowups of toric varieties. This enables us to provide, among other results, an elementary geometric proof of the Laurent phenomenon for cluster algebras (of geometric type), extend Speyer's example [Spe13] of upper cluster algebras which are not finitely generated, and show that the Fock-Goncharov dual basis conjecture is usually false.
\end{abstract}

\section{Contents}

1 Log Calabi-Yau varieties and a geometric motivation for cluster varieties

2 Review of the $\mathcal{X}$ and $\mathcal{A}$ cluster varieties 146

3 The geometry of cluster varieties $\quad \mathbf{1 5 2}$

3.1 Elementary transformations. . . . . . . . . . . . . . . . . . 152

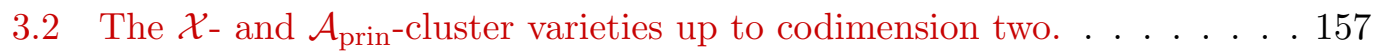

4 The $\mathcal{A}_{t}$ and $\mathcal{A}_{\text {prin }}$ cluster varieties as torsors $\quad \mathbf{1 6 0}$

5 The $\mathcal{X}$ variety in the $\operatorname{rank} \epsilon=2$ case $\quad \mathbf{1 6 5}$

6 Examples of non-finitely-generated upper cluster algebras $\quad \mathbf{1 7 0}$

7 Counterexamples to the Fock-Goncharov dual bases conjecture $\quad 171$

References

173

\section{Introduction}

Cluster algebras were introduced by Fomin and Zelevinsky in [FZ02a]. Fock and Goncharov introduced a more geometric point of view in [FG09], introducing the $\mathcal{A}$ and $\mathcal{X}$ cluster varieties constructed by gluing together "seed tori" via birational maps known as cluster transformations.

In this note, motivated by our study of log Calabi-Yau varieties initiated in the two-dimensional case in [GHK11], we give a simple alternate explanation of basic constructions in the theory of cluster algebras in terms of blowups of toric varieties. Each seed roughly gives a description of the $\mathcal{A}$ or $\mathcal{X}$ cluster variety (more precisely, the $\mathcal{A}_{\text {prin }}, \mathcal{A}_{t}$ (defined in $\S 2$ ) or $\mathcal{X}$ cluster variety) as a blowup of a toric variety, and a mutation of the seed corresponds to changing the blowup description by an elementary transformation of a $\mathbb{P}^{1}$-bundle. Certain global features

Received 20 September 2013, accepted in final form 14 April 2014.

2010 Mathematics Subject Classification 14J32,14M17,14M25

Keywords: cluster algebras, log Calabi-Yau varieties, blowups of toric varieties

This journal is (C) Foundation Compositio Mathematica 2015. This article is distributed with Open Access under the terms of the Creative Commons Attribution Non-Commercial License, which permits non-commercial reuse, distribution, and reproduction in any medium, provided that the original work is properly cited. For commercial re-use, please contact the Foundation Compositio Mathematica.

The first author was partially supported by NSF grant DMS-1105871 and DMS-1262531, the second by NSF grants DMS-0968824 and DMS-1201439, and the third by NSF grant DMS-0854747. 


\section{Mark Gross, Paul Hacking and Sean Keel}

of the cluster variety not obvious from the expression as a union of tori are easily seen from this construction. For example, it gives a simple geometric explanation for the Laurent phenomenon (originally proved in [FZ02b]); see Corollary 3.11. From the blowup picture it is clear that the Fock-Goncharov dual basis conjecture, particularly the statement that tropical points of the Langlands dual $\mathcal{A}$ parameterize a natural basis of regular functions on $\mathcal{X}$, can fail frequently; see $\S 7$.

In more detail, in $\S 1$, we explain the basic philosophical point of view demonstrating how a study of log Calabi-Yau varieties can naturally lead to the basic notions of cluster algebras. This section can be read as an extended introduction; its role in the paper is purely motivational. In $\S 2$, we review the definitions of cluster varieties, following [FG09]. We pay special attention to the precise procedure for gluing tori via cluster transformations, as this has not been discussed to the precision we need in the literature.

Section 3 is the heart of the paper. Here we describe how cluster transformations, which a priori are birational maps between algebraic tori, can be viewed naturally as isomorphisms between blowups of certain associated toric varieties. In this manner, cluster transformations can be interpreted as elementary transformations, a standard procedure for modifying $\mathbb{P}^{1}$-bundles in algebraic geometry. This procedure blows up a codimension two center in a $\mathbb{P}^{1}$-bundle meeting any $\mathbb{P}^{1}$ fibre in at most one point, and blows down the proper transform of the union of $\mathbb{P}^{1}$ fibres meeting the center.

This is a very general construction, covered in $\S 3.1$; we then specialize to the case of the $\mathcal{A}$ and $\mathcal{X}$ cluster varieties in $\S 3.2$. Unfortunately, our construction does not work in general for the $\mathcal{A}$ cluster variety, but does work for the $\mathcal{A}$ variety with principal coefficients. This variety $\mathcal{A}_{\text {prin }}$ fibres over an algebraic torus with $\mathcal{A}$ being the fibre over the identity element of the torus. Properties such as the Laurent phenomenon for $\mathcal{A}$ can be deduced from that for $\mathcal{A}_{\text {prin. }}$. Many of the phenomena discussed here also work for a very general fibre $\mathcal{A}_{t}$ of the map from $\mathcal{A}_{\text {prin }}$; we call such a cluster variety an $\mathcal{A}$ cluster variety with general coefficients. The algebra of regular functions of such a cluster variety is of the kind considered by Speyer in [Spe13].

The key result is Theorem 3.9, which gives the precise description of the $\mathcal{X}$, principal $\mathcal{A}$ cluster varieties and $\mathcal{A}$ cluster varieties with general coefficients up to codimension two in terms of a blowup of a toric variety. The toric variety and the center of the blowup is specified very directly by the seed data determining the cluster variety. An immediate consequence is the Laurent phenomenon, Corollary 3.11.

In $\S 4$, we give another description of the principal $\mathcal{A}$ cluster variety and $\mathcal{A}$ cluster variety with general coefficients in terms of line bundles on the $\mathcal{X}$ cluster variety. There is in fact an algebraic torus which acts on $\mathcal{A}_{\text {prin }}$, and the quotient of this action is $\mathcal{X}$, making $\mathcal{A}_{\text {prin }}$ a torsor of $\mathcal{X}$. We give a precise description of this family in terms of line bundles on $\mathcal{X}$. Furthermore, there are tori $T_{K^{*}}$ and $T_{K^{\circ}}$ such that there are a map $\mathcal{X} \rightarrow T_{K^{*}}$ and an action of $T_{K^{\circ}}$ on any $\mathcal{A}$ cluster variety with general coefficients determined by the seed data. We show that for any such sufficiently general $\mathcal{A}$ cluster variety $\mathcal{A}_{t}$, there is a $\phi=\phi(t) \in T_{K^{*}}$ such that up to codimension two, $\mathcal{A}_{t}$ is the universal torsor of $\mathcal{X}_{\phi}$, essentially obtained as $\operatorname{Spec} \bigoplus_{\mathcal{L} \in \operatorname{Pic}\left(\mathcal{X}_{\phi}\right)} \mathcal{L}$. In particular, this allows us to identify the corresponding upper cluster algebra with the Cox ring of $\mathcal{X}_{\phi}$. This is a slight simplification of the discussion: see the main text for precise statements. The Cox ring of any variety with finitely generated torsion free Picard group is factorial; see [Arz09] and [BH03]. This explains the ubiquity of factorial cluster algebras remarked on, for example, in [Kel12, § 4.6].

The remainder of the paper now restricts to the case that the skew-symmetric matrix determining the cluster algebra has rank two. This case is quite easy to interpret geometrically, 


\section{BirAtionAl GEOMETRY OF CLUSTER ALGEBRAS}

since now the family $\mathcal{X} \rightarrow T_{K^{*}}$ is a family of surfaces. In fact, the fibres are essentially the interiors of Looijenga pairs. A Looijenga pair is a pair $(Y, D)$ where $Y$ is a rational surface and $D \in\left|-K_{Y}\right|$ is a cycle of rational curves, $U:=Y \backslash D$ is the interior. We study moduli of such pairs in [GHK15]. Here, we show (Theorem 5.5) that essentially $\mathcal{X} \rightarrow T_{K^{*}}$ coincides with a type of universal family constructed in [GHK15]. Our construction implies that in many cases, the kernel of the skew-symmetric matrix carries a canonical symmetric form, invariant under mutations; see Theorem 5.6. Though not (as far as we know) previously observed, this symmetric form controls the gross geometry of $\mathcal{X}$, in particular the generic fibre of $\mathcal{X} \rightarrow T_{K^{*}}$. Indeed, the fibre is affine if and only if the form is negative definite; when the form is indefinite the fibres are the complement of a single point in a compact complex analytic space, and thus have no non-constant global functions. Thus in this indefinite case (which from the blowup point of view is the generic situation) the only global functions on $\mathcal{X}$ are pulled back from $T_{K^{*}}$, contradicting the dual basis conjecture of [FG09]; see $\S 7$.

In $\S 6$, we give a general procedure for constructing upper cluster algebras with general or principal coefficients which are not finitely generated. These examples generalize that given by Speyer in [Spe13], and suggest that "most" upper cluster algebras are not finitely generated. These examples arise because Cox rings tend not to be finitely generated. Indeed, finite generation of the Cox ring of a projective variety is a very strong (Mori Dream Space) condition; see [HK00].

For the genesis of this article we refer to the Acknowledgements at the end of this paper.

In this paper, we will always work over a field $\mathbb{k}$ of characteristic zero.

\section{Log Calabi-Yau varieties and a geometric motivation for cluster varieties}

To a geometer, at least to the three of us, the definition of a cluster algebra is rather bizarre and overwhelming. Here we explain the geometric motivation in terms of log Calabi-Yau varieties. There are two elementary constructions of log Calabi-Yau (CY) varieties. The first method is to glue together tori in such a way that the volume forms patch. The second method is to blow up a toric variety along a codimension two center which is a smooth divisor in a boundary divisor, and then remove the strict transform of the toric boundary. As we will see, the simplest instances of either construction are closely related, and either leads to cluster varieties. The first approach extends the viewpoint of [FG09], the second was inspired by [Loo81].

DeFinition 1.1. Let $(Y, D)$ be a smooth projective variety with a normal crossings divisor, and let $U=Y \backslash D$. By [Iit77], the vector subspace

$$
H^{0}\left(Y, \omega_{Y}(D)^{\otimes m}\right) \subset H^{0}\left(U, \omega_{U}^{\otimes m}\right)
$$

(where the inclusion is induced by restriction) depends only on $U$, that is, is independent of the choice of normal crossings compactification. We say that $U$ is $\log$ Calabi-Yau (log CY) if for all $m$ this subspace is one-dimensional, generated by $\Omega^{\otimes m}$ for a volume (that is, nowhere vanishing) form $\Omega \in H^{0}\left(U, \omega_{U}\right)$. Note that by definition $\Omega$ is unique up to scaling.

In practice, log CY varieties are often recognized using the following lemma.

Lemma 1.2. Let $(Y, D)$ be a dlt pair with $K_{Y}+D$ trivial (in particular Cartier) and $Y$ projective. Let $U \subset Y \backslash D$ be a smooth open subset, with $(Y \backslash D) \backslash U$ of codimension at least two. Then $U$ is $\log C Y$.

For the definition of dlt (divisorial log terminal); see [KM98, Definition 2.37]. As this section should be viewed as purely motivational, the reader who wishes to avoid the technicalities of 


\section{Mark Gross, Paul Hacking and Sean Keel}

the minimal model program should feel free to assume that the pair $(Y, D)$ has in fact normal crossings.

Proof. When $(Y, D)$ has normal crossings this is immediate from Definition 1.1. The definition of dlt is such that the vector space of Definition 1.1 can be computed using a dlt (instead of normal crossings) compactification.

Remark 1.3. The data $(Y, D)$ with $U \subseteq Y \backslash D$ as in the lemma is called a minimal model for $U$. One example of a minimal model is a pair $(Y, D)$ with $D \in\left|-K_{Y}\right|$ a reduced normal crossings divisor. This is a minimal model for $U=Y \backslash D$. The main conjectures of the minimal model program would imply every log CY has a minimal model; see [BCHM10].

Lemma 1.4. (i) Let $U \subset V$ be an open subset, with $(U, \Omega) \log C Y$. Then $V$ is $\log C Y$ if and only if $\Omega$ extends to a volume form on $V$, and in this case $\Omega$ is a scalar multiple of the volume form of $V$.

(ii) Let $\mu: U \rightarrow V$ be a birational map between smooth varieties which is an isomorphism outside codimension two subsets of the domain and range. Then $U$ is $\log C Y$ if and only if $V$ is.

Proof. For statement (i), if $V$ is $\log \mathrm{CY}$, then clearly its volume form restricts to a scalar multiple of the volume form on $U$. Now suppose $U$ is $\log \mathrm{CY}$, and its volume form $\Omega$ extends to a volume form on $V$. We have $U \subseteq V \subseteq Y$ where $Y$ is a compactification of both $U$ and $V$. Thus $\Omega$ (and its powers) obviously has at worst simple poles on any divisor contained in $Y \backslash V$, and it is unique in this respect, since we have the same properties for $\Omega$ as a volume form on $U$. Next, statement (ii) follows from statement (i), passing to the open subsets where the map is an isomorphism, noting that in statement (i), when the complement of $U$ has codimension at least two, the extension condition is automatic.

Definition 1.5. We say a log CY $U$ has maximal boundary if it has a minimal model $(Y, D)$ with a zero-dimensional log canonical center. For example, this is the case if $(Y, D)$ is a minimal model for $U$ such that $D$ has simple normal crossings and contains a zero-dimensional stratum, that is, a point which is the intersection of $\operatorname{dim}(Y)$ distinct irreducible components of $D$.

Example 1.6. Consider the group $G=\mathrm{PGL}_{n}$. There are the $2 n-1$ minors of an $n \times n$ matrix given by the square submatrices in the upper right corner or the lower left corner. For example, for $n=3$ these are the four minors

$$
a_{1,3}, a_{3,1},\left|\begin{array}{ll}
a_{1,2} & a_{1,3} \\
a_{2,2} & a_{2,3}
\end{array}\right|,\left|\begin{array}{ll}
a_{2,1} & a_{2,2} \\
a_{3,1} & a_{3,2}
\end{array}\right|
$$

and the determinant of the $3 \times 3$ matrix itself. Let $D \subset Y=\mathbb{P}\left(\mathrm{Mat}_{n \times n}\right)=\mathbb{P}^{n^{2}-1}$ be the union of the $2 n-1$ divisors given by the zero locus of these minors. Note the total degree of $D$ is

$$
1+2+\cdots+(n-1)+1+2+\cdots+(n-1)+n=n^{2},
$$

so $D \in\left|-K_{Y}\right|$. With some non-trivial effort, one can check $(Y, D)$ is dlt, with a zero-dimensional $\log$ canonical center, and thus $(Y, D)$ is a minimal model for the smooth affine log CY with maximal boundary $U \subset G$, the non-vanishing locus of this collection of minors. $U$ is by definition the open double Bruhat cell in $G$.

A $\log$ CY $U$ with maximal boundary will (in dimension at least two) always have infinitely many minimal models. The set of possibilities leads to a fundamental invariant, as follows. 


\section{Birational GEOMETRY OF CLUSTER ALGEBRAS}

Definition 1.7. Let $(U, \Omega)$ be a log CY. Define

$$
\begin{aligned}
U^{\text {trop }}(\mathbb{Z}) & :=\{\text { divisorial discrete valuations } v: k(U) \backslash\{0\} \rightarrow \mathbb{Z} \mid v(\Omega)<0\} \cup\{0\} \\
& :=\left\{(E, m) \mid m \in \mathbb{Z}_{+}, E \subset(Y \backslash U), \Omega \text { has a pole along } E\right\} \cup\{0\} .
\end{aligned}
$$

Here $k(U)$ is the field of rational functions of $U$, a discrete valuation is called divisorial if it is given by the order of vanishing of a divisor on some variety birational to $U$. Furthermore, we define

$$
v\left(g d z_{1} \wedge \cdots \wedge d z_{n}\right):=v(g)
$$

for $z_{1}, \ldots, z_{n}$ local coordinates in a neighborhood of the generic point of the divisor corresponding to $v$; this is independent of the choice of coordinates as a change of coordinates only changes $g$ by a unit. In the second expression $E$ is a divisorial irreducible component of the boundary in some partial compactification $U \subset Y$, and two divisors on two possibly different birational varieties are identified if they give the same valuation on their common field of fractions.

The simplest example of a log CY with maximal boundary is an algebraic torus

$$
T_{N}:=N \otimes \mathbb{G}_{m},
$$

for $N=\mathbb{Z}^{n}$. Note $H^{0}\left(T_{N}, \mathcal{O}_{T_{N}}\right)=\mathbb{k}[M]$, where $M=\operatorname{Hom}(N, \mathbb{Z})$ is the character lattice of $T_{N}$.

LEMMA 1.8. Restriction of valuations to the character lattice $M$ induces a canonical isomorphism

$$
T_{N}^{\mathrm{trop}}(\mathbb{Z})=N
$$

A minimal model for $T_{N}$ is the same as a complete $T_{N}$-equivariant toric compactification.

Proof. This is an easy log discrepancy computation, using, for example, [KM98, Lemmas 2.29 and 2.45].

Thus $U^{\text {trop }}(\mathbb{Z})$ gives an analog for any log CY of the cocharacter lattice of a torus. Note however that in general $U^{\operatorname{trop}}(\mathbb{Z})$ is not a group as addition does not make sense. We conjecture that there is also an analog of the character lattice, or equivalently, the dual torus.

Conjecture 1.9 [GHK11]. Let $(Y, D)$ be a simple normal crossings minimal model for a log CY with maximal boundary $U=Y \backslash D$, and assume $D$ supports an ample divisor (note that this implies $U$ is affine). Let $R=\mathbb{k}\left[\operatorname{Pic}(Y)^{*}\right]$. The free $R$-module $V$ with basis $U^{\operatorname{trop}}(\mathbb{Z})$ has a natural finitely generated $R$-algebra structure whose structure constants are non-negative integers determined by counts of rational curves on $U$. The associated fibration $p: \operatorname{Spec}(V) \rightarrow \operatorname{Spec}(R)=$ $T_{\text {Pic }(Y)}$ is a flat family of affine log CY varieties with maximal boundary. Letting $K$ be the kernel of the natural surjection $\operatorname{Pic}(Y) \rightarrow \operatorname{Pic}(U), p$ is $T_{K}$-equivariant. The quotient family $\operatorname{Spec}(V) / T_{K} \rightarrow T_{\operatorname{Pic}(U)}$ depends only on $U$ (is independent of the choice of minimal model), and is the mirror family to $U$.

Remark 1.10. An analog of Conjecture 1.9 is expected for compact Calabi-Yau varieties, but perhaps only with formal (for example, Novikov) parameters, and for Calabi-Yau varieties near the so-called large complex structure limit. This will be discussed in forthcoming work. The maximal boundary condition means the boundary is highly degenerate — we are thus already in some sense in the large complex structure limit, and so one can hope that no formal power series or further limits are required. This is one reason to focus on this case. The other is the wealth of fundamental examples. 


\section{Mark Gross, Paul Hacking and Sean Keel}

The conjecture is of interest independently of mirror symmetry: in many instances the variety $U$ and its prospective mirror are known varieties of compelling interest. The conjecture then gives a new construction of a variety we already care about, a construction which in particular endows the mirror (and each fibre of the family) with a canonical basis of functions. In any case mirror symmetry is conjecturally an involution, the mirror of the mirror being a family of deformations of the original $U$. Thus the conjecture says in particular that any affine $\log \mathrm{CY}$ with maximal boundary is a fibre of the output of such a construction, and thus in particular has a canonical basis of functions, $B_{U}$. One then expects $B_{U}$ to be the tropical set of the conjectural mirror.

We call a partial compactification $U \subset Y$ a partial minimal model if the volume form $\Omega$ has a pole on every irreducible divisorial component of $Y \backslash U$. One checks using Lemma 1.8 that a partial minimal model for an algebraic torus is the same thing as a toric variety. We further conjecture that for any partial minimal model (not necessarily affine) of an affine log CY $U$ with maximal boundary, $B_{U} \cap H^{0}\left(Y, \mathcal{O}_{Y}\right) \subset H^{0}\left(Y, \mathcal{O}_{Y}\right)$ is a basis of regular functions on $Y$. For example we conjecture that the open double Bruhat cell $U \subset G$ has a canonical basis of functions, and that the subset of basis elements which extend regularly to $G$ gives a basis of functions on $G$.

After tori, the next simplest example of a $\log \mathrm{CY}$ with maximal boundary is obtained by gluing together algebraic tori in such a way that the volume forms patch. More precisely, suppose that

$$
\mathcal{A}=\bigcup_{\mathbf{s} \in \mathcal{S}} T_{N, \mathbf{s}}
$$

is a variety covered by open copies of the torus $T_{N}$ indexed by the set $\mathcal{S}$. This gives canonical birational maps $\mu_{\mathbf{s}, \mathbf{s}^{\prime}}: T_{N, \mathbf{s}} \rightarrow T_{N, \mathbf{s}^{\prime}}$ for each pair of seeds $\mathbf{s}, \mathbf{s}^{\prime} \in \mathcal{S}$. Then $\mathcal{A}$ will be $\log$ CY if and only if each birational map is a mutation, that is, preserves the volume form: $\mu^{*}(\Omega)=\Omega$. In this case each choice of seed torus $T_{N, \mathbf{s}} \subset \mathcal{A}$ gives a canonical identification $\mathcal{A}^{\text {trop }}(\mathbb{Z})=T_{N, \mathbf{s}}^{\text {trop }}(\mathbb{Z})=N$.

We can reverse the procedure. Beginning with a collection of such mutations satisfying the cocycle condition, we can canonically glue together the tori along the maximal open sets where the maps are isomorphisms to form a $\log \mathrm{CY} \mathcal{A}$. See Proposition 2.4 for details. The simplest example of a mutation comes from a pair $(n, m) \in N \times M$ with $\langle n, m\rangle:=m(n)=0$. It is defined by

$$
\mu_{(n, m)}^{*}\left(z^{m^{\prime}}\right)=z^{m^{\prime}} \cdot\left(1+z^{m}\right)^{\left\langle m^{\prime}, n\right\rangle}
$$

where $z^{m^{\prime}}, z^{m} \in \mathbb{k}[M]$ are the corresponding characters of $T_{N}$. Cluster varieties are log CY varieties formed by gluing tori by mutations of this simple sort (and compositions of such) for a particular parameterizing set $\mathcal{S}$. See $\S 2$ for details.

Though these are the simplest non-toric log CY varieties, there are already very interesting examples, including double Bruhat cells for reductive groups, their flag varieties and unipotent radicals, and character varieties of punctured Riemann surfaces. See [BFZ05] and [FG06].

Note that these simple mutations come in obvious dual pairs — we can simply reverse the order and consider

$$
(m,-n) \in \operatorname{Hom}(N \times M, \mathbb{Z})=M \times N,
$$

so that $\mu_{(m,-n)}$ defines a birational automorphism of $T_{M}$. Thus for each $\mathcal{A}:=\bigcup_{\mathbf{s} \in \mathcal{S}} T_{N, \mathbf{s}}$ built from such maps, there is a canonical $d u a l \mathcal{X}:=\bigcup_{\mathbf{s} \in \mathcal{S}} T_{M, \mathbf{s}}$, just obtained by replacing each torus (and each mutation) by its dual. For the particular parameterizing set $\mathcal{S}$ used in cluster varieties, Fock and Goncharov made the following remarkable conjecture. 


\section{BiRATIONAL GEOMETRY OF CLUSTER ALGEBRAS}

Conjecture 1.11. The set $\mathcal{A}^{\text {trop }}(\mathbb{Z})$ parameterizes a canonical vector space basis for $H^{0}\left(\mathcal{X}, \mathcal{O}_{\mathcal{X}}\right)$. The structure constants for the algebra $H^{0}\left(\mathcal{X}, \mathcal{O}_{\mathcal{X}}\right)$ expressed in this basis are non-negative integers.

(Here we are treating the notationally simpler case of skew-symmetric cluster varieties, the general case involving a Langlands dual seed.)

Note that as stated $\mathcal{A}$ and $\mathcal{X}$ are on completely equal footing, so the conjecture includes the analogous statement with the two reversed. Fock and Goncharov have a different definition of, for example, $\mathcal{A}^{\text {trop }}(\mathbb{Z})$, which they denote $\mathcal{A}\left(\mathbb{Z}^{t}\right)$, as points of $\mathcal{A}$ valued in the tropical semi-field. But it is easy to check that this agrees with our definition, which has the advantage that it makes sense for any $\log$ CY, while theirs is restricted to varieties with a so-called positive atlas of tori.

In somewhat more detail, a skew-symmetric cluster variety is defined using initial data of a lattice $N$ with a skew-symmetric form $\{\cdot, \cdot\}: N \times N \rightarrow \mathbb{Z}$, and each mutation is given by the pair $(n,\{n, \cdot\})$ for some $n \in N$. When $\{\cdot, \cdot\}$ fails to be unimodular, the dual $M$ does not have a skew-symmetric form, and in this case $\mathcal{A}$ and $\mathcal{X}$ are on unequal footing. In this case $\mathcal{A}$, by the Laurent phenomenon, always has many global functions, but $\mathcal{X}$ may have very few.

Conjecture 1.11 was inspired by the case $\mathcal{A}:=U \subset G$ of Example 1.6, which has a celebrated canonical basis of global functions constructed by G. Lusztig; see [Lus90]. Conjecture 1.9 suggests that the existence of this basis may have a priori nothing to do with representation theory, or cluster varieties, but is rather a general feature of affine $\log$ CY varieties with maximal boundary.

In $\S 7$ we show that Conjecture 1.11 as stated is often false. But if we add the condition that $\mathcal{X}$ is affine, it becomes a very special case of Conjecture 1.9, and for that reason we refer to $\mathcal{X}, \mathcal{A}$ as Fock-Goncharov mirrors. In view of the highly involved existing proposals for synthetic constructions of mirror varieties, [KS06], [GS11], [GHK11], this simple alternative - replace each torus in the open cover by its dual - is an attractive surprise. We will prove many instances of Conjecture 1.11 in [GHKK14].

We now turn to the main idea in this paper, which connects the traditional description above of cluster varieties via gluing tori to the description we will develop in this paper, involving blowups of toric varieties. Here is some cluster motivation for the blowup approach. Each seed $\mathbf{s}$ gives a torus open subset $T_{N, \mathbf{s}} \subset \mathcal{A}$, together with $n$ cluster variables, a basis of characters. These give a priori rational functions on $\mathcal{A}$ and thus a birational map $b: \mathcal{A} \rightarrow \mathbb{A}^{n}$, whose inverse restricts to an isomorphism of the structure torus $\mathbb{G}_{m}^{n} \subset \mathbb{A}^{n}$ with $T_{N, \mathbf{s}} \subset \mathcal{A}$. The Laurent phenomenon is equivalent to the statement that $b$ is regular, and thus in particular suggests that each seed determines a construction of $\mathcal{A}$ as (an open subset of) a blowup of a toric variety (in fact $\mathbb{A}^{n}$ ) along a locus in the toric boundary. Stated this way, it is natural to wonder whether it holds for $\mathcal{X}$ as well. We will show this indeed holds for $\mathcal{X}$, and while it fails for general $\mathcal{A}$, a slightly weaker version is true which is still good enough for the Laurent phenomenon.

Log CY varieties with maximal boundary are closed under blowup in the following sense.

Lemma 1.12. Let $\bar{U} \subset \bar{Y}$ be a $\log C Y$ open subset of a smooth (not necessarily complete) variety, let $\bar{D}:=\bar{Y} \backslash \bar{U}$ and let $H \subset \bar{D} \backslash \operatorname{Sing}(\bar{D})$ be a smooth codimension two (not necessarily irreducible) subvariety. Let $b: Y \rightarrow \bar{Y}$ be the blowup along $H$, let $D \subset Y$ be the strict transform of $\bar{D}$ and let $U:=Y \backslash D$. Then $U$ is $\log C Y$, with unique volume form the pullback under $b$ of the volume form on $\bar{U}$. In addition, $U$ has maximal boundary if $\bar{U}$ does.

Proof. If $E$ is the exceptional divisor of $b$, then it is standard that $K_{Y}=b^{*} K_{\bar{Y}}+E$ (using the fact that $H$ has codimension two) and that $D=b^{*} \bar{D}-E$. Thus $K_{Y}+D=0$. 


\section{Mark Gross, Paul Hacking and Sean Keel}

Now starting with the simplest example, an algebraic torus, we get many examples via the following notion.

Definition 1.13. Continuing with notation as in the lemma, we say that $U=Y \backslash D$ is a cluster $\log C Y$ and that $b:(Y, D) \rightarrow(\bar{Y}, \bar{D})$ is a toric model for $U$ if

(i) the variety $(\bar{Y}, \bar{D})$ is toric and the fan for $\bar{Y}$ consists only of one-dimensional cones $\mathbb{R}_{\geqslant 0} v_{i}$ for $v_{i} \in N$ primitive, with $T_{N}$ the structure torus of $\bar{Y}$ (equivalently, the boundary $D$ is a disjoint union of codimension one tori);

(ii) the connected components of $H$ are the subtori $z^{w_{i}}+1=0 \subset T_{N / \mathbb{Z} \cdot v_{i}}$ for some $w_{i} \in$ $\left(N / \mathbb{Z} \cdot v_{i}\right)^{*}=v_{i}^{\perp} \subset M$.

As the name suggests, the log CY varieties obtained by this simple blowup construction and those obtained in the previous discussion as tori glued in the simplest way are frequently the same. Note that the toric model determines a canonical torus open subset

$$
T_{N}=\bar{Y} \backslash \bar{D} \subset Y \backslash D=U \text {. }
$$

Remarkably, there are (usually infinitely) many other torus open sets. Given a toric model for $U$, and a choice of a center, that is, a connected component of $H$, or equivalently, a choice of one of the primitive lattice points $v=v_{k}$, there is a natural mutation which produces a new $\log \mathrm{CY} U^{\prime}$, with a birational map $U \rightarrow U^{\prime}$. Under certain conditions, this map will be an isomorphism outside a codimension two subset (of domain and range). In these nice situations, this produces, up to codimension two, a second copy of $T_{N}$ living in $U$. Iterating the procedure produces an atlas of torus open sets. Here is a sketch; full details are given in $\S 3$.

The connection with mutation of seeds comes via the tropical set. Note that a mutation $\mu: U \rightarrow V$ between $\log \mathrm{CY}$ varieties canonically induces an isomorphism of tropical sets

$$
\mu^{t}: U^{\operatorname{trop}}(\mathbb{Z}) \rightarrow V^{\operatorname{trop}}(\mathbb{Z}), \quad v \rightarrow v \circ \mu^{*} .
$$

For the mutation $\mu_{(n, m)}: T_{N} \rightarrow T_{N}$ of $(1.2)$, one computes

$$
\mu_{(n, m)}^{t}: N=T_{N}^{\text {trop }}(\mathbb{Z}) \rightarrow T_{N}^{\text {trop }}(\mathbb{Z})=N, \quad \mu^{t}\left(n^{\prime}\right)=n^{\prime}+\left[\left\langle m, n^{\prime}\right\rangle\right]_{-} n,
$$

where for a real number $r,[r]_{-}:=\min (r, 0)$. This illustrates the general fact that $\mu^{t}$ is piecewise linear but not linear (unless $\mu$ is an isomorphism). This explains the geometric origin of piecewise linear maps in the cluster theory (and tropical geometry; see [HKT09, §2]). Here, we view $U^{\operatorname{trop}}(\mathbb{Z})$ as a collection of valuations. If we think of elements of $U^{\operatorname{trop}}(\mathbb{Z})$ as boundary divisors with integer weight, as in the second formula in (1.1), $\mu^{t}$ is simply a strict transform (also called pushforward) for the birational map $\mu$.

Now we explain how to mutate from one toric model of a cluster log CY to another. Continuing with the situation of Definition 1.13, we choose one index, $k$, and let $v=v_{k}$, with corresponding divisor $D_{k}$. The center $H_{k}=H \cap D_{k}$ determines what is known as an elementary transformation in algebraic geometry. We explain this in a simplified, but key, situation.

Let $\Sigma_{v}$ be the fan, with two rays, with support $\mathbb{R} v$, so that the corresponding toric variety $X_{\Sigma_{v}}$ is isomorphic to $T_{N / \mathbb{Z} v} \times \mathbb{P}^{1}$, with $\pi: X_{\Sigma_{v}} \rightarrow T_{N / \mathbb{Z} v}$ the projection. Write $D_{ \pm}$for the two toric divisors corresponding to the rays generated by $\pm v$. Viewing $X_{\Sigma_{v}} \backslash D_{-}$as an open subset of $\bar{Y}$, the center $H_{k}$ is identified with a codimension two subscheme $H_{+} \subset D_{+} \subset X_{\Sigma_{v}}$. Let $H_{-}=\pi^{-1}\left(\pi\left(H_{+}\right)\right) \cap D_{-}$.

There is then a birational map $\mu: X_{\Sigma_{v}} \rightarrow X_{\Sigma_{v}}$ obtained by blowing up $H_{+}$and then blowing down the strict transform of $\pi^{-1}\left(\pi\left(H_{+}\right)\right)$. One checks that $\mu$ is described by (1.2). Clearly by 


\section{BiRATIONAL GEOMETRY OF CLUSTER ALGEBRAS}

construction $\mu$ is resolved by the blowup $b: Y^{\prime} \rightarrow X_{\Sigma_{v}}$ along $H_{+}$, and one can check that $\mu \circ b: Y^{\prime} \rightarrow X_{\Sigma_{v}}$ is regular as well, being the blowup along $H_{-}$; see Lemma 3.2.

This description of the elementary transformation extends to give birational maps between closely related toric models. For simplicity assume $-v \neq v_{i}$ for any $i$ (in $\S 3$ we consider the general case). Now let $\Sigma_{+}$be the fan consisting of rays $\mathbb{R}_{\geqslant 0} v_{i}$ together with $-\mathbb{R}_{\geqslant 0} v$. The toric model gives us a blowup $b: Y \rightarrow X_{\Sigma_{+}}$. (This is a slight abuse of notation, because we added one ray, $-\mathbb{R}_{\geqslant 0} v$. But note that we do not blow up along the new boundary divisor $D_{-} \subset X_{\Sigma_{+}}$, and in forming $U$ we throw away the strict transform of boundary divisors, so adding this ray does not change $U$ at all). Let $\Sigma_{-}$be the fan with rays $\mathbb{R}_{\geqslant 0} \mu^{t}\left(v_{i}\right)$ together with $-\mathbb{R}_{\geqslant 0} v=-\mathbb{R}_{\geqslant 0} \mu^{t}(v)$. In $\S 3.2$ we show that in good situations,

$$
b^{\prime}:=\mu \circ b: Y \rightarrow X_{\Sigma_{-}}
$$

is regular outside a codimension two subset and give formulae for the centers, which again are of the cluster log CY sort. Thus the elementary transformation induces a new toric model for $U$ (up to changes in codimension two), and, in particular, a second torus open subset of $U$. This recovers the standard definition of mutations for cluster algebras [FZ02a]. From this perspective, each seed is interpreted as the data for a toric model of the same (up to codimension two) cluster $\log \mathrm{CY}$. Note that in the mutated toric model $b^{\prime}: Y \rightarrow X_{\Sigma_{-}}$, there is now a center in the boundary divisor $D_{-}$, but no center in $D_{+}$. In the original model $b: Y \rightarrow X_{\Sigma_{+}}$, there is a center in $D_{+} \subset X_{\Sigma_{+}}$(this divisor is the strict transform of $D_{+} \subset X_{\Sigma_{-}}$) but no center in $D_{-} \subset X_{\Sigma_{+}}$. For all the other boundary divisors, there is a center in either model. This difference between the chosen index $k$ and the other indices accounts for the peculiar sign change in the formula for seed mutation; see (2.3).

Unfortunately, this procedure does not always give a precise identification between the picture of cluster varieties as obtained from gluing of tori and the picture given by blowups of toric varieties. The reason is that $b^{\prime}$ above need not always be regular off a codimension two subset. It turns out that this works in certain cases, including all $\mathcal{X}$ cluster varieties and principal $\mathcal{A}$ cluster varieties. See $\S 2$ for a review of the definitions of the latter, and $\S 3$ for further details.

Remark 1.14. There is no need to restrict to the special centers of Definition 1.13(2): one can consider the blowup of an arbitrary hypersurface in each boundary divisor. An elementary transform gives a mutation of a toric model in the same way, but the formulae for how the centers change are more complicated. For a general center, we checked that one obtains the mutation formulae of [LP12]. In this note we restrict our treatment to the cluster variety case, as it is simpler and sufficient for our applications.

There are many formulae in the Fomin-Zelevinsky, Fock-Goncharov definitions of cluster algebras, which we reproduce in the next section. But we note that only one, (1.2), is essential. This is the birational mutation, $\mu$, between tori in the $\mathcal{A}$-atlas. Its canonical dual, arising from (1.3), gives the mutation for the Fock-Goncharov mirror; see (2.6) and (2.5) below. The formula for the change of seed, (2.3), comes from the tropicalization, $\mu^{t}$, of the birational mutation, (1.4). Note that in (2.3), $e_{i}^{\prime}=\mu^{t}\left(e_{i}\right)$ for $i \neq k$ and $e_{k}^{\prime}=\mu^{t}\left(-e_{k}\right)=-e_{k}$. This is the peculiar sign change explained above. 


\section{Mark Gross, Paul Hacking and Sean Keel}

\section{Review of the $\mathcal{X}$ and $\mathcal{A}$ cluster varieties}

We follow [FG09], with minor modifications. We will fix once and for all in the discussion the following data, which we will refer to as fixed ${ }^{1}$ data:

- a lattice $N$ with a skew-symmetric bilinear form $\{\cdot, \cdot\}: N \times N \rightarrow \mathbb{Q}$;

- an unfrozen sublattice $N_{\text {uf }} \subseteq N$, a saturated sublattice of $N$; if $N_{\text {uf }}=N$, we say the fixed data has no frozen variables;

- an index set $I$ with $|I|=\operatorname{rank} N$ and a subset $I_{\mathrm{uf}} \subseteq I$ with $\left|I_{\mathrm{uf}}\right|=\operatorname{rank} N_{\mathrm{uf}}$;

- positive integers $d_{i}$ for $i \in I$ with greatest common divisor 1 ;

- a sublattice $N^{\circ} \subseteq N$ of finite index such that $\left\{N_{\text {uf }}, N^{\circ}\right\} \subseteq \mathbb{Z},\left\{N, N_{\text {uf }} \cap N^{\circ}\right\} \subseteq \mathbb{Z}$;

- $M=\operatorname{Hom}(N, \mathbb{Z}), M^{\circ}=\operatorname{Hom}\left(N^{\circ}, \mathbb{Z}\right)$.

Given this fixed data, seed data for this fixed data is a labeled collection of elements of $N$

$$
\mathbf{s}:=\left(e_{i} \mid i \in I\right)
$$

such that $\left\{e_{i} \mid i \in I\right\}$ is a basis for $N,\left\{e_{i} \mid i \in I_{\mathrm{uf}}\right\}$ a basis for $N_{\mathrm{uf}}$, and $\left\{d_{i} e_{i} \mid i \in I\right\}$ is a basis for $N^{\circ}$.

A choice of seed data $\mathbf{s}$ defines a new (non-skew-symmetric) bilinear form on $N$ by

$$
[\cdot, \cdot]_{\mathbf{s}}: N \times N \rightarrow \mathbb{Q}, \quad\left[e_{i}, e_{j}\right]_{\mathbf{s}}=\epsilon_{i j}:=\left\{e_{i}, e_{j}\right\} d_{j}
$$

Note that $\epsilon_{i j} \in \mathbb{Z}$ as long as we do not have $i, j \in I \backslash I_{\mathrm{uf}}$. This bilinear form depends on the seed. We drop the subscript $\mathbf{s}$ if it is obvious from the context.

Remark 2.1. Suppose that we specify a basis $e_{i}, i \in I$ for a lattice $N, I_{\mathrm{uf}} \subseteq I$, positive integers $d_{i}$ and a matrix $\epsilon_{i j}$ satisfying $d_{i} \epsilon_{i j}=-d_{j} \epsilon_{j i}$ and $\epsilon_{i j} \in \mathbb{Z}$ provided we do not have $i, j \in I \backslash I_{\mathrm{uf}}$. This data determines the data $N, N_{\mathrm{uf}}, N^{\circ},\{\cdot, \cdot\}$, etc. It will turn out that $\epsilon_{i j}$ for $i, j \in I \backslash I_{\mathrm{uf}}$ does not affect the schemes we construct, and it is standard in the literature to just consider rectangular matrices $\left(\epsilon_{i j}\right)_{i \in I_{\mathrm{uf}}, j \in I}$. We wish, however, to emphasize that the fixed data does not depend on the particular choice of seed.

Given a seed $\mathbf{s}$, we obtain a dual basis $\left\{e_{i}^{*}\right\}$ for $M$ and a basis $\left\{f_{i}\right\}$ of $M^{\circ}$ given by $f_{i}=d_{i}^{-1} e_{i}^{*}$. We use the notation

$$
\langle\cdot, \cdot\rangle: N \times M^{\circ} \rightarrow \mathbb{Q}
$$

for the canonical pairing given by evaluation. We also write for $i \in I_{\mathrm{uf}}, v_{i}:=\left\{e_{i}, \cdot\right\} \in M^{\circ}$. We have two natural maps defined by $\{\cdot, \cdot\}$ :

$$
\begin{array}{ll}
p_{1}^{*}: N_{\mathrm{uf}} \rightarrow M^{\circ} & p_{2}^{*}: N \rightarrow M^{\circ} / N_{\mathrm{uf}}^{\perp} \\
N_{\mathrm{uf}} \ni n \mapsto\left(N^{\circ} \ni n^{\prime} \mapsto\left\{n, n^{\prime}\right\}\right) & N \ni n \mapsto\left(N_{\mathrm{uf}} \cap N^{\circ} \ni n^{\prime} \mapsto\left\{n, n^{\prime}\right\}\right)
\end{array}
$$

For the future, let us choose a map

$$
p^{*}: N \rightarrow M^{\circ}
$$

such that $\left.p^{*}\right|_{N_{\mathrm{uf}}}=p_{1}^{*}$ and the composed map $N \rightarrow M^{\circ} / N_{\mathrm{uf}}^{\perp}$ agrees with $p_{2}^{*}$. Different choices $^{2}$ of $p^{*}$ differ by a choice of map $N / N_{\mathrm{uf}} \rightarrow N_{\mathrm{uf}}^{\perp}$.

\footnotetext{
${ }^{1}$ This terminology is not standard in the cluster literature. Rather, what we call fixed data along with seed data is referred to as seed data in the literature. We prefer to distinguish the data which remains unchanged under mutation from the data which changes.

${ }^{2}$ We note that [FG09] gives an incorrect definition when $N_{\text {uf }} \neq N$, as the formula $p^{*}(n)=\{n, \cdot\}$ may not give a result in $M^{\circ}$.
} 


\section{Birational GEOMETRY OF CLUSTER ALGEBRAS}

Given seed data $\mathbf{s}$, we can associate two tori

$$
\mathcal{X}_{\mathbf{s}}=T_{M}=\operatorname{Spec} \mathbb{k}[N] \quad \text { and } \quad \mathcal{A}_{\mathbf{s}}=T_{N^{\circ}}=\operatorname{Spec} \mathbb{k}\left[M^{\circ}\right] .
$$

We write $X_{1}, \ldots, X_{n}$ as coordinates on $\mathcal{X}_{\mathbf{s}}$ corresponding to the basis vectors $e_{1}, \ldots, e_{n}$, that is, $X_{i}=z^{e_{i}}$, and similarly coordinates $A_{1}, \ldots, A_{n}$ corresponding to the basis vectors $f_{1}, \ldots, f_{n}$, that is, $A_{i}=z^{f_{i}}$. The coordinates $X_{i}, A_{i}$ are called cluster variables. These coordinates give identifications

$$
\mathcal{X}_{\mathbf{s}} \rightarrow \mathbb{G}_{m}^{n}, \quad \mathcal{A}_{\mathbf{s}} \rightarrow \mathbb{G}_{m}^{n} .
$$

We write these two split tori as $\left(\mathbb{G}_{m}^{n}\right)_{X}$ and $\left(\mathbb{G}_{m}^{n}\right)_{A}$ in the $\mathcal{X}$ and $\mathcal{A}$ cases, respectively.

Remark 2.2. These tori come with the following structures.

(i) Let $K=\operatorname{ker} p_{2}^{*}$; then the inclusion $K \subseteq N$ induces a map $\mathcal{X}_{\mathbf{s}} \rightarrow T_{K^{*}}=\operatorname{Spec} \mathbb{k}[K]$. Furthermore, the torus $T_{\left(N / N_{\mathrm{uf}}\right)^{*}}=\operatorname{Spec} \mathbb{k}\left[N / N_{\mathrm{uf}}\right]$ is a subtorus of $\mathcal{X}_{\mathrm{s}}$ and hence acts on $\mathcal{X}_{\mathbf{s}}$.

(ii) Let $K^{\circ}=K \cap N^{\circ}$; then the inclusion $K^{\circ} \rightarrow N^{\circ}$ induces a map of tori $T_{K^{\circ}} \rightarrow \mathcal{A}_{\mathbf{s}}$. This gives an action of $T_{K^{\circ}}$ on $\mathcal{A}_{\mathbf{s}}$. Furthermore, there is a natural inclusion

$$
N_{\mathrm{uf}}^{\perp}=\left\{m \in M^{\circ} \mid\langle m, n\rangle=0 \quad \forall n \in N_{\mathrm{uf}}\right\} \subseteq M^{\circ} .
$$

This induces a map $\mathcal{A}_{\mathbf{s}} \rightarrow T_{N^{\circ} / N_{\mathrm{uf}} \cap N^{\circ}}=\operatorname{Spec} \mathbb{k}\left[N_{\mathrm{uf}}^{\perp}\right]$.

(iii) The chosen map $p^{*}: N \rightarrow M^{\circ}$ defines a map $p: \mathcal{A}_{\mathbf{s}} \rightarrow \mathcal{X}_{\mathbf{s}}$. Furthermore, $p^{*}$ induces maps $p^{*}: K \rightarrow N_{\mathrm{uf}}^{\perp} \subseteq M^{\circ}$ and $p^{*}: N / N_{\mathrm{uf}} \rightarrow\left(K^{\circ}\right)^{*}$, giving maps

$$
p: T_{N^{\circ} / N_{\mathrm{uf}} \cap N^{\circ}} \rightarrow T_{K^{*}} \quad \text { and } \quad p: T_{K^{\circ}} \rightarrow T_{\left(N / N_{\mathrm{uf}}\right)^{*}},
$$

respectively. We then obtain commutative diagrams
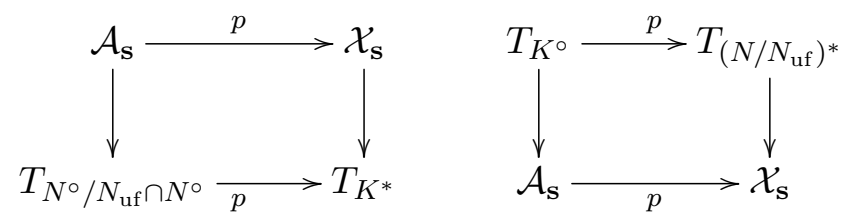

We next define a mutation of seed data.

For $r \in \mathbb{Q}$ define $[r]_{+}=\max (0, r)$. Given seed data $\mathbf{s}$ and $k \in I_{\mathrm{uf}}$, we have a mutation $\mu_{k}(\mathbf{s})$ of $\mathbf{s}$ given by a new basis

$$
e_{i}^{\prime}:= \begin{cases}e_{i}+\left[\epsilon_{i k}\right]_{+} e_{k} & \text { for } i \neq k, \\ -e_{k} & \text { for } i=k .\end{cases}
$$

Note that $\left\{e_{i}^{\prime} \mid i \in I_{\mathrm{uf}}\right\}$ still form a basis for $N_{\mathrm{uf}}$ and the $d_{i} e_{i}^{\prime}$ still form a basis for $N^{\circ}$. Dually, one checks that the basis $\left\{f_{i}\right\}$ for $M^{\circ}$ changes as

$$
f_{i}^{\prime}:= \begin{cases}-f_{k}+\sum_{j}\left[-\epsilon_{k j}\right]_{+} f_{j} & \text { for } i=k \\ f_{i} & \text { for } i \neq k .\end{cases}
$$

One also checks that the matrix $\epsilon_{i j}$ changes via the formula

$$
\epsilon_{i j}^{\prime}:=\left\{e_{i}^{\prime}, e_{j}^{\prime}\right\} d_{j}= \begin{cases}-\epsilon_{i j} & \text { for } k \in\{i, j\}, \\ \epsilon_{i j} & \text { for } \epsilon_{i k} \epsilon_{k j} \leqslant 0 \text { and } k \notin\{i, j\}, \\ \epsilon_{i j}+\left|\epsilon_{i k}\right| \epsilon_{k j} & \text { for } \epsilon_{i k} \epsilon_{k j}>0 \text { and } k \notin\{i, j\} .\end{cases}
$$




\section{Mark Gross, Paul Hacking and Sean Keel}

We also define birational maps

$$
\begin{aligned}
& \mu_{k}: \mathcal{X}_{\mathbf{s}} \rightarrow \mathcal{X}_{\mu_{k}(\mathbf{s})} \\
& \mu_{k}: \mathcal{A}_{\mathbf{s}} \rightarrow-\rightarrow \mathcal{A}_{\mu_{k}(\mathbf{s})}
\end{aligned}
$$

via pull-back of functions

$$
\begin{aligned}
\mu_{k}^{*} z^{n}=z^{n}\left(1+z^{e_{k}}\right)^{-\left[n, e_{k}\right]} & \text { for } n \in N, \\
\mu_{k}^{*} z^{m}=z^{m}\left(1+z^{v_{k}}\right)^{-\left\langle d_{k} e_{k}, m\right\rangle} & \text { for } m \in M^{\circ} .
\end{aligned}
$$

These maps are more often seen in the cluster literature as described via pull-backs of cluster variables:

$$
\mu_{k}^{*} X_{i}^{\prime}= \begin{cases}X_{k}^{-1} & \text { for } i=k, \\ X_{i}\left(1+X_{k}^{-\operatorname{sgn}\left(\epsilon_{i k}\right)}\right)^{-\epsilon_{i k}} & \text { for } i \neq k\end{cases}
$$

and

$$
A_{k} \cdot \mu_{k}^{*} A_{k}^{\prime}=\prod_{j: \epsilon_{k j}>0} A_{j}^{\epsilon_{k j}}+\prod_{j: \epsilon_{k j}<0} A_{j}^{-\epsilon_{k j}}, \quad \mu_{k}^{*} A_{i}^{\prime}=A_{i} \quad \text { for } i \neq k .
$$

The correspondence between these two descriptions can be seen using $X_{i}=z^{e_{i}}, X_{i}^{\prime}=z^{e_{i}^{\prime}}$ and $A_{i}=z^{f_{i}}, A_{i}^{\prime}=z^{f_{i}^{\prime}}$.

Remark 2.3. Note that in the notation of (1.2), the mutation (2.6) is

$$
\mu_{\left(-d_{k} e_{k}, v_{k}\right)}: T_{N \circ-\rightarrow} T_{N \circ} .
$$

By (1.4), its tropicalization is

$$
\mu_{k}^{t}(n)=n+\left[\left\langle v_{k}, n\right\rangle\right]_{-}\left(-d_{k} e_{k}\right)=n+\left[\left\{n, d_{k} e_{k}\right\}\right]_{+} e_{k},
$$

and thus the seed mutation (2.3) is also given by

$$
e_{i}^{\prime}= \begin{cases}\mu_{k}^{t}\left(e_{i}\right) & \text { for } i \neq k, \\ -e_{k}=-\mu_{k}^{t}\left(e_{k}\right) & \text { for } i=k .\end{cases}
$$

On the other hand, the mutation (2.5) is $\mu_{\left(d_{k} v_{k}, e_{k}\right)}: T_{M} \rightarrow T_{M}$. This tropicalizes to

$$
\mu_{k}^{t}(m)=m+\left[\left\langle d_{k} e_{k}, m\right\rangle\right]_{-} v_{k} .
$$

Note that as $p^{*}$ is a linear function, the $v_{i}$ transform under the mutation in the same way the $e_{i}$ do; that is, $v_{k}^{\prime}=-v_{k}, v_{i}^{\prime}=v_{i}+\left[\epsilon_{i k}\right]_{+} v_{k}$ for $i \neq k$. But $\mu_{k}^{t}\left(v_{i}\right)=v_{i}+\left[\epsilon_{i k}\right]_{-} v_{k} \neq v_{i}^{\prime}$, so we do not obtain an equation analogous to (2.9). Rather, one checks that

$$
-v_{i}^{\prime}= \begin{cases}\mu_{k}^{t}\left(-v_{i}\right) & \text { for } i \neq k \\ -\mu_{k}^{t}\left(-v_{k}\right) & \text { for } i=k\end{cases}
$$

One checks easily the commutativity of the diagrams

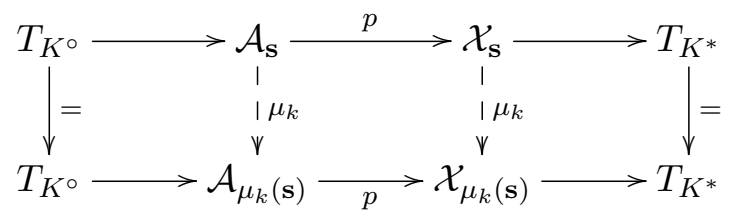



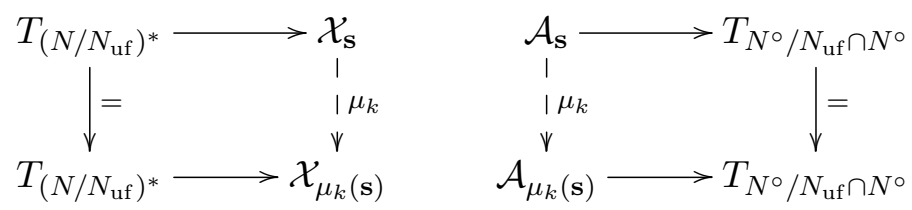

We can now define the $\mathcal{X}$ and $\mathcal{A}$ cluster varieties associated with the seed $\mathbf{s}$. We will first need the following general gluing construction.

Proposition 2.4. Let $\left\{X_{i}\right\}$ be a collection of integral, separated schemes of finite type over a field $\mathbb{k}$, with birational maps $f_{i j}: X_{i} \rightarrow X_{j}$ for all $i, j$, with $f_{i i}$ the identity and $f_{j k} \circ f_{i j}=f_{i k}$ as rational maps. Let $U_{i j} \subseteq X_{i}$ be the largest open subset such that $f_{i j}: U_{i j} \rightarrow f_{i j}\left(U_{i j}\right)$ is an isomorphism. Then there is a scheme $X$ obtained by gluing the $X_{i}$ along the open sets $U_{i j}$ via the maps $f_{i j}$.

Proof. First, the sets $U_{i j}$ exist: take $U_{i j}$ to consist of all points $x$ in the domain of $f_{i j}$ at which $f_{i j}$ is a local isomorphism. By [Gro60, 6.5.4], these are precisely the points $x$ such that $f_{i j}^{*}$ : $\mathcal{O}_{X_{j}, f_{i j}(x)} \rightarrow \mathcal{O}_{X_{i}, x}$ is an isomorphism. By [Gro60, 8.2.8], $\left.f_{i j}\right|_{U_{i j}}$ is an open immersion.

By [Har77, Exercise II 2.12], it is now sufficient to check that $f_{i j}\left(U_{i j} \cap U_{i k}\right)=U_{j i} \cap U_{j k}$. Clearly $f_{i j}\left(U_{i j} \cap U_{i k}\right) \subseteq U_{j i}$. If $x \in U_{i j} \cap U_{i k}$, then $f_{j k}$ can be defined at $f_{i j}(x) \in U_{j i}$ via $f_{i k} \circ f_{i j}^{-1}$. Then, clearly $f_{j k}$ is a local isomorphism at $f_{i j}(x)$, so $f_{i j}(x) \in U_{j k}$. Conversely, if $y \in U_{j i} \cap U_{j k}$, then $y=f_{i j}(x)$ for some $x \in U_{i j}$. Clearly $f_{i k}=f_{j k} \circ f_{i j}$ is a local isomorphism at $x$, so $x \in U_{i k}$ also and $y \in f_{i j}\left(U_{i j} \cap U_{i k}\right)$.

Let $\mathfrak{T}$ be the oriented rooted tree with $\left|I_{\mathrm{uf}}\right|$ outgoing edges from each vertex, labeled by the elements of $I_{\text {uf }}$. Let $v$ be the root of the tree. Attach the seed $\mathbf{s}$ to the vertex $v$. Now, each simple path starting at $v$ determines a sequence of seed mutations, just mutating at the label attached to the edge. In this way we attach a seed to each vertex of $\mathfrak{T}$. We write the seed attached to a vertex $w$ as $\mathbf{s}_{w}$. We further attach copies $\mathcal{X}_{\mathbf{s}_{w}}, \mathcal{A}_{\mathbf{s}_{w}}$ to $w$.

If $\mathfrak{T}$ has a directed edge from $w$ to $w^{\prime}$ labeled with $k \in I_{\mathrm{uf}}$, with associated seeds $\mathbf{s}_{w}$ and

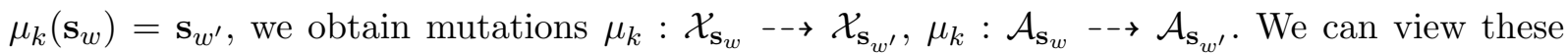
maps as arising from traversing the edge in the direction from $w$ to $w^{\prime}$; we use the inverse maps $\mu_{k}^{-1}$ if we traverse the edge from $w^{\prime}$ to $w$.

Now for any two vertices $w, w^{\prime}$ of $\mathfrak{T}$, there is a unique simple path $\gamma$ from one to the other. We obtain birational maps

$$
\mu_{w, w^{\prime}}: \mathcal{A}_{\mathbf{s}_{w}} \rightarrow \mathcal{A}_{\mathbf{s}_{w^{\prime}}}, \quad \mu_{w, w^{\prime}}: \mathcal{X}_{\mathbf{s}_{w}} \rightarrow \mathcal{X}_{\mathbf{s}_{w^{\prime}}}
$$

between the associated tori. These are obtained by taking the composition of mutations or their inverses associated with each edge traversed by $\gamma$ in the order traversed, using a mutation $\mu_{k}$ associated with the edge if the edge is traversed in the direction of its orientation, and using $\mu_{k}^{-1}$ if the edge is traversed in the opposite direction.

These birational maps clearly satisfy $\mu_{w^{\prime}, w^{\prime \prime}} \circ \mu_{w, w^{\prime}}=\mu_{w, w^{\prime \prime}}$ as birational maps; hence, by Proposition 2.4, we obtain schemes $\mathcal{X}$ or $\mathcal{A}$ by gluing these tori using these birational maps.

Remark 2.5. Note that $\mu_{k} \circ \mu_{k}: \mathcal{A}_{\mathbf{s}} \rightarrow \mathcal{A}_{\mu_{k}\left(\mu_{k}(\mathbf{s})\right)}$ is not the identity when expressed as a map $\operatorname{Spec} \mathbb{k}\left[M^{\circ}\right] \rightarrow \operatorname{Spec} \mathbb{k}\left[M^{\circ}\right]$; rather, it is the isomorphism given by the linear map $M^{\circ} \rightarrow M^{\circ}$, $m \mapsto m-\left\langle d_{k} e_{k}, m\right\rangle v_{k}$. This map takes the basis $\left\{f_{i}\right\}$ for the seed $\mu_{k}\left(\mu_{k}(\mathbf{s})\right)$ to the basis $\left\{f_{i}\right\}$ for the seed s. This is why $\mu_{k} \circ \mu_{k}$ is only the identity when viewed as an automorphism of Spec $\mathbb{k}\left[A_{1}^{ \pm 1}, \ldots, A_{n}^{ \pm 1}\right]$. 


\section{Mark Gross, Paul Hacking and Sean Keel}

Remark 2.6. As we shall see in Theorem 3.14, the $\mathcal{A}$ variety is always separated, but the $\mathcal{X}$ variety usually is not. It is not clear, however, whether either of these schemes is Noetherian. This will sometimes cause problems in what follows, but these problems are purely technical. In particular, given any finite connected regular subtree $\mathfrak{T}^{\prime}$ of $\mathfrak{T}$, we can use the seed tori corresponding to vertices in $\mathfrak{T}^{\prime}$ to define open subschemes of $\mathcal{X}$ and $\mathcal{A}$. We shall write these subschemes as $\mathcal{X}^{\mathrm{ft}}$ and $\mathcal{A}^{\mathrm{ft}}$ respectively. We will not need to be particularly concerned about which subtree $\mathfrak{T}^{\prime}$ we use, only that it be sufficiently big for the purpose at hand. However, we shall always assume that $\mathfrak{T}^{\prime}$ contains the root vertex $v$ and all its adjacent vertices.

Remark 2.7. The structures (i)-(iii) of Remark 2.2 described on individual seed tori, being compatible with mutations as seen in (2.11) and (2.12), induce corresponding structure on $\mathcal{X}$ and $\mathcal{A}$. In particular,

(i) there are a canonical map $\lambda: \mathcal{X} \rightarrow T_{K^{*}}$ and a canonical action of $T_{\left(N / N_{\mathrm{uf}}\right)^{*}}$ on $\mathcal{X}$;

(ii) there are a canonical action of $T_{K^{\circ}}$ on $\mathcal{A}$ and a canonical map $\mathcal{A} \rightarrow T_{N^{\circ} / N_{\mathrm{uf}} \cap N^{\circ}}$;

(iii) there is a map $p: \mathcal{A} \rightarrow \mathcal{X}$; this map is compatible with the maps and actions of items (i) and (ii) as indicated in Remark 2.2(iii).

Definition 2.8. The $\mathcal{X}$-cluster algebra $\left(\mathcal{A}\right.$-cluster algebra) associated with a seed $\mathbf{s}$ is $\Gamma\left(\mathcal{X}, \mathcal{O}_{\mathcal{X}}\right)$ $\left(\right.$ or $\Gamma\left(\mathcal{A}, \mathcal{O}_{\mathcal{A}}\right)$ ).

Remark 2.9. The $\mathcal{A}$-cluster algebra is usually called the upper cluster algebra in the literature; see [BFZ05]. This can be viewed as the algebra of Laurent polynomials in $\mathbb{k}\left[M^{\circ}\right]$ which remain Laurent polynomials under any sequence of mutations. Such a Laurent polynomial is called a universal Laurent polynomial. The algebra which is usually just called the cluster algebra is the sub-algebra of the field of fractions $k\left(\mathcal{A}_{\mathbf{s}}\right)=\mathbb{k}\left(A_{1}, \ldots, A_{n}\right)$ of $\mathcal{A}_{\mathbf{s}}$ generated by all functions

$$
\left\{\mu_{v, w}^{*}\left(A_{i}^{\prime}\right) \mid A_{i}^{\prime} \text { is a coordinate on } \mathcal{A}_{\mathbf{s}_{w}}, w \text { a vertex of } \mathfrak{T}\right\} \text {. }
$$

We note that the cluster algebras arising via this construction are still a special case of the general definition given in [FZ02a], and are called cluster algebras of geometric type in the literature. These include most of the important examples.

We end this section with several variants of the constructions above.

Construction 2.10. When there are frozen variables (that is, $N_{\mathrm{uf}} \neq N$ ) one frequently might want to allow the frozen variables $X_{i}, i \notin I_{\mathrm{uf}}$ or $A_{i}, i \notin I_{\mathrm{uf}}$ to take the value zero. Thus, one replaces $\mathcal{X}_{\mathbf{s}}, \mathcal{A}_{\mathbf{s}}$ with

$$
\begin{aligned}
& \mathcal{X}_{\mathbf{s}}:=\operatorname{Spec} \mathbb{k}\left[\left\{X_{i}^{ \pm 1} \mid i \in I_{\mathrm{uf}}\right\} \cup\left\{X_{i} \mid i \notin I_{\mathrm{uf}}\right\}\right], \\
& \mathcal{A}_{\mathbf{s}}:=\operatorname{Spec} \mathbb{k}\left[\left\{A_{i}^{ \pm 1} \mid i \in I_{\mathrm{uf}}\right\} \cup\left\{A_{i} \mid i \notin I_{\mathrm{uf}}\right\}\right] .
\end{aligned}
$$

These varieties can be defined somewhat more abstractly as toric varieties, with fans the set of faces of the cone generated by $\left\{e_{i}^{*} \mid i \notin I_{\mathrm{uf}}\right\}$ and $\left\{d_{i} e_{i} \mid i \notin I_{\mathrm{uf}}\right\}$, respectively. One sees from (2.7) and (2.8) that no $X_{i}$ or $A_{i}$ for $i \notin I_{\mathrm{uf}}$ is inverted by mutations. Thus cluster varieties $\mathcal{X}$, $\mathcal{A}$ can be defined via gluing these modified spaces as before. In particular, we obtain a map $\mathcal{A} \rightarrow \operatorname{Spec} \mathbb{k}\left[\left\{A_{i} \mid i \notin I_{\mathrm{uf}}\right\}\right]$.

In any event, Fock and Goncharov [FG11] define the special completion of the $\mathcal{X}$ variety, written as $\widehat{\mathcal{X}}$, by replacing each $\mathcal{X}_{\mathbf{s}}$ with the affine space Spec $\mathbb{k}\left[X_{1}, \ldots, X_{n}\right]$ and using the same definition for the birational maps between the $\mathcal{X}_{\mathbf{s}}$ as usual. 


\section{Birational GeOMETRy of Cluster Algebras}

Construction 2.11. We define the notion of cluster algebra with principal coefficients. In general, given fixed data $N,\{\cdot, \cdot\}$ as usual along with seed data $\mathbf{s}$, we construct the double of the lattice via

$$
\widetilde{N}=N \oplus M^{\circ}, \quad\left\{\left(n_{1}, m_{1}\right),\left(n_{2}, m_{2}\right)\right\}=\left\{n_{1}, n_{2}\right\}+\left\langle n_{1}, m_{2}\right\rangle-\left\langle n_{2}, m_{1}\right\rangle .
$$

We take $\widetilde{N}_{\text {uf }}=N_{\text {uf }} \subseteq \widetilde{N}$ and $\widetilde{N}^{\circ}$ the sublattice $N^{\circ} \oplus M$. The lattice $\widetilde{N}$ with its pairing $\{\cdot, \cdot\}$ and sublattices $\widetilde{N}_{\text {uf }}, \widetilde{N}^{\circ}$ can now play the role of fixed data. Given a seed $\mathbf{s}$ for the original fixed data, we obtain a seed $\tilde{\mathbf{s}}$ for $\widetilde{N}$ with basis $\left\{\left(e_{i}, 0\right),\left(0, f_{\alpha}\right)\right\}$. We use the convention that indices $i, j, k \in I$ are used to index the first set of basis elements and $\alpha, \beta, \gamma \in I$ are used to index the second set of basis elements. The integer $d_{i}$ associated with $\left(e_{i}, 0\right)$ or $d_{\alpha}$ associated with $\left(0, f_{\alpha}\right)$ is then taken to agree with $d_{i}$ or $d_{\alpha}$ of the original seed. Then the matrix $\tilde{\epsilon}$ determined by this seed is given by

$$
\tilde{\epsilon}_{i j}=\epsilon_{i j}, \quad \tilde{\epsilon}_{i \beta}=\delta_{i \beta}, \quad \tilde{\epsilon}_{\alpha j}=-\delta_{\alpha j}, \quad \tilde{\epsilon}_{\alpha \beta}=0 .
$$

One notes that $\widetilde{M}=\operatorname{Hom}(\tilde{N}, \mathbb{Z})=M \oplus N^{\circ}$ and $\widetilde{M}^{\circ}=M^{\circ} \oplus N$. Furthermore, given a choice of $p^{*}: N \rightarrow M^{\circ}$, we can take the map $p^{*}: \widetilde{N} \rightarrow \widetilde{M}^{\circ}$ to be given by

$$
p^{*}\left(e_{i}, 0\right)=\left(p^{*}\left(e_{i}\right), e_{i}\right), \quad p^{*}\left(0, f_{\alpha}\right)=\left(-f_{\alpha}, 0\right)
$$

so that $p^{*}$ is an isomorphism.

With this choice of fixed and seed data, the corresponding $\mathcal{A}$ cluster variety will be written as $\mathcal{A}_{\text {prin. }}$. The ring of global functions on $\mathcal{A}_{\text {prin }}$ is the upper cluster algebra with principal coefficients at the seed s of [FZ07, Definition 3.1].

The variety $\mathcal{A}_{\text {prin }}$ has an additional relationship with $\mathcal{X}$. There are two natural inclusions,

$$
\begin{aligned}
\tilde{p}^{*}: N & \rightarrow \widetilde{M}^{\circ} & \pi^{*}: N & \rightarrow M^{\circ} \\
n & \mapsto\left(p^{*}(n), n\right) & n & \mapsto(0, n)
\end{aligned}
$$

The first inclusion induces for any seed $\mathbf{s}$ an exact sequence of tori

$$
1 \longrightarrow T_{N^{\circ}} \longrightarrow \mathcal{A}_{\text {prin }, \mathbf{s}} \stackrel{\tilde{p}}{\longrightarrow} \mathcal{X}_{\mathbf{s}} \longrightarrow 1 \text {. }
$$

One checks that $\tilde{p}$ commutes with the mutations $\mu_{k}$ on $\mathcal{A}_{\text {prin,s }}$ and $\mathcal{X}_{\mathbf{s}}$. Thus, we obtain a morphism $\tilde{p}: \mathcal{A}_{\text {prin }} \rightarrow \mathcal{X}$. The $T_{N^{\circ}}$ action on $\mathcal{A}_{\text {prin,s }}$ gives a $T_{N^{\circ}}$ action on $\mathcal{A}_{\text {prin }}$, making $\tilde{p}$ the

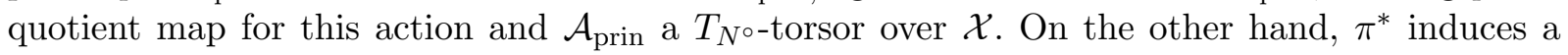
projection

$$
\pi: \mathcal{A}_{\text {prin }} \rightarrow T_{M}
$$

We note that if $e \in T_{M}$ denotes the identity element, then $\pi^{-1}(e)=\mathcal{A}$. To see this, note that the fibre of $\pi: \mathcal{A}_{\text {prin,s }} \rightarrow T_{M}$ over $e$ is canonically $\mathcal{A}_{\mathbf{s}}$, and a mutation $\mu_{k}$ on $\mathcal{A}_{\text {prin,s }}$ specializes to the corresponding mutation on $\mathcal{A}_{\mathbf{s}}$. The open subset on which a mutation $\mu_{w, w^{\prime}}: \mathcal{A}_{\text {prin, } \mathbf{s}_{w}} \rightarrow \mathcal{A}_{\text {prin, } \mathbf{s}_{w^{\prime}}}$ is an isomorphism onto its image restricts to the corresponding open subset of $\mathcal{A}_{\mathbf{s}_{w}}$; otherwise, $\mathcal{A}_{\text {prin }}$ would not be separated, contradicting Theorem 3.14.

Definition 2.12. Let $t \in T_{M}$. We write $\mathcal{A}_{t}$ for the fibre $\pi^{-1}(t)$. We call this an $\mathcal{A}$ cluster variety with general coefficients.

Construction 2.13. In case there are no frozen variables, that is, $N=N_{\text {uf }}$, we have $p^{*}=p_{2}^{*}$ 


\section{Mark Gross, Paul Hacking and Sean Keel}

and $K=\operatorname{ker} p^{*}$. We then have a commutative diagram

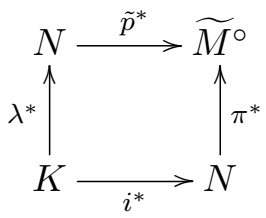

where both $i^{*}$ and $\lambda^{*}$ are the inclusion. This induces a commutative diagram

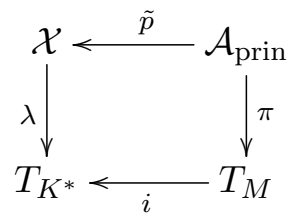

Note that for $t \in T_{M}, \tilde{p}$ restricts to a map $p_{t}: \mathcal{A}_{t} \rightarrow \lambda^{-1}(i(t))=\mathcal{X}_{i(t)}$.

\section{The geometry of cluster varieties}

We now give our description of cluster varieties as blowups of toric varieties and mutations as elementary transformations of $\mathbb{P}^{1}$-bundles. This gives rise to most of the results in this paper, including a simple explanation for the Laurent phenomenon and counterexamples to some basic conjectures about cluster algebras.

\subsection{Elementary transformations.}

The basic point is that the gluing of adjacent seed tori can be easily described in terms of blowups of toric varieties, and that mutations have a simple interpretation as a well-known operation in algebraic geometry known as an elementary transformation. To describe this in general, we fix a lattice $N$ with no additional data and a primitive vector $v \in N$. The projection $N \rightarrow N / \mathbb{Z} v$ gives a $\mathbb{G}_{m}$-bundle $\pi: T_{N} \rightarrow T_{N / \mathbb{Z} v}$. A non-zero regular function $f$ on $T_{N / \mathbb{Z} v}$ can be viewed as a map

$$
\begin{aligned}
f: T_{N / \mathbb{Z} v} \backslash V(f) & \rightarrow T_{\mathbb{Z} v} \subseteq T_{N}=N \otimes_{\mathbb{Z}} \mathbb{G}_{m} \\
t & \mapsto v \otimes f(t)
\end{aligned}
$$

to obtain a birational map

$$
\begin{aligned}
\mu_{f}: T_{N} & \longrightarrow T_{N} \\
t & \longmapsto f(\pi(t))^{-1} \cdot t .
\end{aligned}
$$

Note that on the level of pull-back of functions, this is defined, for $m \in M=\operatorname{Hom}(N, \mathbb{Z})$, by

$$
z^{m} \mapsto z^{m}(f \circ \pi)^{-\langle m, v\rangle} .
$$

Indeed, this is easily checked by choosing a basis $f_{1}, \ldots, f_{n}$ of $M$ with $\left\langle f_{1}, v\right\rangle=1,\left\langle f_{i}, v\right\rangle=0$ for $i>1$. This gives coordinates $x_{i}=z^{f_{i}}, 1 \leqslant i \leqslant n$, on $T_{N}$, so that the projection $\pi$ is given by $\left(x_{1}, \ldots, x_{n}\right) \mapsto\left(x_{2}, \ldots, x_{n}\right)$, and the map $\mu_{f}$ is given by

$$
\left(x_{1}, \ldots, x_{n}\right) \mapsto\left(f\left(x_{2}, \ldots, x_{n}\right)^{-1} x_{1}, x_{2}, \ldots, x_{n}\right) .
$$

Now consider the fan $\Sigma_{v,+}=\left\{\mathbb{R}_{\geqslant 0} v, 0\right\}$ in $N$. This defines a toric variety $\operatorname{TV}\left(\Sigma_{v,+}\right)$ isomorphic to $\mathbb{A}^{1} \times T_{N / \mathbb{Z} v}$ and containing a toric divisor $D_{+}$. It has a canonical projection 


\section{Birational GEOMETRY OF CLUSTER ALGEBRAS}

$\pi: \operatorname{TV}\left(\Sigma_{v,+}\right) \rightarrow T_{N / \mathbb{Z} v}$, which induces an isomorphism $D_{+} \cong T_{N / \mathbb{Z} v}$. Set

$$
Z_{+}=\pi^{-1}(V(f)) \cap D_{+} .
$$

This hypersurface may be non-reduced. Define

$$
\begin{aligned}
& \widetilde{\mathrm{TV}}\left(\Sigma_{v,+}\right) \rightarrow \operatorname{TV}\left(\Sigma_{v,+}\right) \text { the blowup of } Z_{+}, \\
& \tilde{D}_{+} \text {the proper transform of } D_{+} \text {and } U_{v,+}=\widetilde{\mathrm{TV}}\left(\Sigma_{v,+}\right) \backslash \tilde{D}_{+} .
\end{aligned}
$$

Note that $\Gamma\left(U_{v,+}, \mathcal{O}_{U_{v,+}}\right)=\Gamma\left(\operatorname{TV}\left(\Sigma_{v,+}\right), \mathcal{O}_{\mathrm{TV}\left(\Sigma_{v,+}\right)}\right)\left[f / x_{1}\right]$.

We can also use $\mu_{f}$ to define a variety $X_{f}$ obtained by gluing together two copies of $T_{N}$ using $\mu_{f}$ along the open subsets $T_{N} \backslash V(f \circ \pi) \subseteq T_{N}$.

We then obtain the following basic model for describing gluings of tori as blowups of toric varieties.

Lemma 3.1. There is an open immersion $X_{f} \hookrightarrow U_{v,+}$ such that $U_{v,+} \backslash X_{f}$ is of codimension two in $U_{v,+}$. Furthermore, the projection $\pi: U_{v,+} \rightarrow T_{N / \mathbb{Z} v}$ is a $\mathbb{G}_{m}$-bundle over $T_{N / \mathbb{Z} v} \backslash V(f)$, while the fibres of $\pi$ over $V(f)$ are each a union of two copies of $\mathbb{A}^{1}$ meeting at a point. The locus where $\pi$ is not smooth is precisely $U_{v,+} \backslash X_{f}$.

Proof. Using coordinates $\left(x_{1}, \ldots, x_{n}\right)$ for $\mathrm{TV}\left(\Sigma_{v,+}\right)$ as before, with $D_{+}$given by $x_{1}=0$, note that the ideal of $Z_{+}$is $\left(x_{1}, f\right)$. Thus the blowup of $Z_{+}$is given by the equation $u x_{1}=v f$ in $\mathbb{P}^{1} \times \operatorname{TV}\left(\Sigma_{v,+}\right)$. We define two embeddings of $T_{N}$,

$$
\begin{aligned}
& \iota_{1}:\left(x_{1}, \ldots, x_{n}\right) \mapsto\left(\left(f, x_{1}\right),\left(x_{1}, \ldots, x_{n}\right)\right), \\
& \iota_{2}:\left(x_{1}, \ldots, x_{n}\right) \mapsto\left(\left(1, x_{1}\right),\left(x_{1} f, x_{2}, \ldots, x_{n}\right)\right) .
\end{aligned}
$$

Noting that $\mu_{f}=\iota_{2}^{-1} \circ \iota_{1}$, it is clear that these maps give an embedding of $X_{f}$. The divisor $\tilde{D}_{+}$ is given by the equation $v=x_{1}=0$, so the only points of $U_{v,+}$ missed by the open immersion $X_{f} \hookrightarrow U_{v,+}$ are the points where $u=x_{1}=0$, that is, points of the form $\left((0,1),\left(0, x_{2}, \ldots, x_{n}\right)\right)$ with $f\left(x_{2}, \ldots, x_{n}\right)=0$. The remaining statements are clear.

Next we examine how this gives a basic model for a mutation. Consider the fan $\Sigma_{v}:=$ $\left\{\mathbb{R}_{\geqslant 0} v, \mathbb{R}_{\leqslant 0} v, 0\right\}$. This defines a toric variety we write as $\mathbb{P}$, which comes with divisors $D_{+}, D_{-}$ corresponding to the two rays and a map

$$
\pi: \mathbb{P} \rightarrow T_{N / \mathbb{Z} v}
$$

identifying $D_{+}$and $D_{-}$with $T_{N / \mathbb{Z} v}$. Let

$$
\begin{aligned}
& Z_{+}=D_{+} \cap V(f \circ \pi), \\
& Z_{-}=D_{-} \cap V(f \circ \pi) .
\end{aligned}
$$

We have two blowups

$$
b_{ \pm}: \tilde{\mathbb{P}}_{ \pm} \rightarrow \mathbb{P}
$$

that are the blowups of $Z_{+}$and $Z_{-}$.

LEMma 3.2. The rational map $\mu_{f}: T_{N} \rightarrow T_{N}$ extends to a regular isomorphism $\mu_{f}: \tilde{\mathbb{P}}_{+} \rightarrow \tilde{\mathbb{P}}_{-}$.

Proof. Working in coordinates $\left(x_{1}, \ldots, x_{n}\right)$ as before, we can describe $\mathbb{P}$ as $\mathbb{P}^{1} \times T_{N / \mathbb{Z} v}$ with coordinates $\left(x_{1}: y_{1}\right)$ on $\mathbb{P}^{1}$ and coordinates $x_{2}, \ldots, x_{n}$ on $T_{N / \mathbb{Z} v}$. Here $D_{+}$is given by $x_{1}=0$ and $D_{-}$by $y_{1}=0$. Then $\mu_{f}$ is given as

$$
\left(\left(x_{1}: y_{1}\right),\left(x_{2}, \ldots, x_{n}\right)\right) \mapsto\left(\left(x_{1}, f\left(x_{2}, \ldots, x_{n}\right) y_{1}\right),\left(x_{2}, \ldots, x_{n}\right)\right) .
$$




\section{Mark Gross, Paul Hacking and Sean Keel}

This fails to be defined precisely where $x_{1}=f=0$, that is, along $Z_{+}$; blowing up $Z_{+}$clearly resolves this indeterminacy. Thus, $\mu_{f}: \mathbb{P} \rightarrow \mathbb{P}$ lifts to a morphism $\mu_{f}: \tilde{\mathbb{P}}_{+} \rightarrow \mathbb{P}$. On the other hand, since the ideal sheaf of $Z_{-}$in $\mathbb{P}$ (locally generated by $y_{1}$ and $f$ ) pulls back via $\mu_{f}$ to an invertible sheaf on $\tilde{\mathbb{P}}_{+}$, this morphism factors as a morphism $\mu_{f}: \tilde{\mathbb{P}}_{+} \rightarrow \tilde{\mathbb{P}}_{-}$by the universal property of blowing up.

To see that $\mu_{f}$ viewed this way is a regular isomorphism, note that the inverse rational map $\mu_{f}^{-1}$ can be written as $t \mapsto f(\pi(t)) \cdot t$, and thus as a map $\mathbb{P} \rightarrow \mathbb{P}$ is written as

$$
\left(\left(x_{1}: y_{1}\right),\left(x_{2}, \ldots, x_{n}\right)\right) \mapsto\left(\left(f\left(x_{2}, \ldots, x_{n}\right) x_{1}, y_{1}\right),\left(x_{2}, \ldots, x_{n}\right)\right) .
$$

This lifts to a well-defined morphism $\mu_{f}^{-1}: \tilde{\mathbb{P}}_{-} \rightarrow \tilde{\mathbb{P}}_{+}$, as before. Thus $\mu_{f}$ is an isomorphism between $\tilde{\mathbb{P}}_{+}$and $\tilde{\mathbb{P}}_{-}$.

Remark 3.3. This lemma should be interpreted as saying that $\mu_{f}: \mathbb{P} \rightarrow \mathbb{P}$ can be viewed as the birational map described as the blowup of $Z_{+}$followed by the contraction of the proper transform of $\pi^{-1}(V(f)) \subseteq \mathbb{P}$ in $\tilde{\mathbb{P}}_{+}$to $Z_{-} \subseteq \mathbb{P}$. This is a birational operation called an elementary transformation in algebraic geometry.

Furthermore, let $\tilde{D}_{ \pm}$be the proper transform of $D_{ \pm}$in either $\tilde{\mathbb{P}}_{+}$or $\tilde{\mathbb{P}}_{-}$. Then combining Lemmas 3.1 and 3.2 , this tells us that there are open immersions of $X_{f}$ in $\tilde{\mathbb{P}}_{ \pm} \backslash\left(\tilde{D}_{+} \cup \tilde{D}_{-}\right)$, missing a codimension two subset. The roles the two coordinate tori of $X_{f}$ play are reversed under these two immersions; one of the tori of $X_{f}$ is the inverse image of the big torus orbit under the blowup $\tilde{\mathbb{P}}_{-} \rightarrow \mathbb{P}$, while the other torus in $X_{f}$ is the inverse image of the big torus orbit under the blowup $\tilde{\mathbb{P}}_{+} \rightarrow \mathbb{P}$.

We need an extended version of the above setup.

Construction 3.4. Suppose that we have the data of a fan $\Sigma=\left\{\mathbb{R}_{\geqslant 0} v_{i} \mid 1 \leqslant i \leqslant \ell\right\} \cup\{0\}$, where $v_{1}, \ldots, v_{\ell} \in N$ are primitive, and $w_{1}, \ldots, w_{\ell} \in M$ with $\left\langle v_{i}, w_{i}\right\rangle=0$. We allow some of the points $v_{i}$ to coincide. Let $a_{1}, \ldots, a_{\ell}$ be positive integers, let $c_{1}, \ldots, c_{\ell} \in \mathbb{k}^{\times}$, and let $\mu_{i}: T_{N} \rightarrow T_{N}$ be defined as before by the data $f_{i}=\left(1+c_{i} z^{w_{i}}\right)^{a_{i}}$ and $v_{i}$, where $c_{i} \in \mathbb{k}^{\times}$. Let $\operatorname{TV}(\Sigma)$ be the toric variety defined by $\Sigma$, and let $D_{i}$ be the toric divisor corresponding to $\mathbb{R}_{\geqslant 0} v_{i}$.

In what follows, we use the notation $\bar{V}\left(f_{i}\right)$ for the closure of $V\left(f_{i}\right) \subseteq T_{N}$ in $\operatorname{TV}(\Sigma)$. Define

$$
\begin{aligned}
& Z_{j}=D_{j} \cap \bar{V}\left(f_{j}\right), \pi: \widetilde{\mathrm{TV}}(\Sigma) \rightarrow \mathrm{TV}(\Sigma) \text { the blowup along } \bigcup_{i=1}^{\ell} Z_{i} \text { and } \\
& \tilde{D}_{j} \text { the proper transform of } D_{j} .
\end{aligned}
$$

On the other hand, define a scheme $X$ as follows. Let $T_{0}, \ldots, T_{\ell}$ be $\ell+1$ copies of the torus $T_{N}$. The map $\mu_{i}$ is viewed as an isomorphism

$$
\varphi_{0 i}:=\mu_{i}: U_{0 i} \rightarrow U_{i 0}
$$

between open sets of $T_{0}$ and $T_{i}$, respectively, with $U_{0 i}$ taken as the largest possible such open subset. Indeed, we can take $U_{0 i}=T_{0} \backslash V\left(f_{i}\right)$ and $U_{i 0}=T_{i} \backslash V\left(f_{i}\right)$. In addition, for $1 \leqslant i, j \leqslant \ell$, define $\varphi_{i j}:=\mu_{j} \circ \mu_{i}^{-1}$, and define $U_{i j}$ to be the largest subset of $T_{i}$ on which $\varphi_{i j}$ defines an open immersion. The identifications $\varphi_{i j}$ then provide gluing data to obtain a scheme $X$, in general not separated, by Proposition 2.4.

LEMMA 3.5. There is a natural morphism

$$
\psi: X \rightarrow \tilde{U}_{\Sigma}:=\widetilde{\mathrm{TV}}(\Sigma) \backslash \bigcup_{i} \tilde{D}_{i},
$$

which in special cases satisfies the following properties: 


\section{BiRATIONAL GEOMETRY OF CLUSTER ALGEBRAS}

(i) If $\operatorname{dim} Z_{i} \cap Z_{j}<\operatorname{dim} Z_{i}$ for all $i \neq j$, then $\psi$ is an isomorphism outside a set of codimension at least two.

(ii) If $Z_{i} \cap Z_{j}=\emptyset$ for all $i \neq j$, then $\psi$ is an open immersion. In particular, in this case, $X$ is separated.

Proof. This is just a slightly more involved version of the argument of Lemma 3.1. We first describe maps of the tori $T_{i}, 0 \leqslant i \leqslant \ell$, into $\tilde{U}_{\Sigma}$. We have a canonical identification of $T_{0}$ with the big torus orbit $T_{N}$ of $\operatorname{TV}(\Sigma)$, isomorphic to $\pi^{-1}\left(T_{N}\right) \subseteq \tilde{U}_{\Sigma}$. On the other hand, for a given $i$, let $J$ be the set of indices such that $v_{j}=v_{i}$ if and only if $j \in J$. Note that $\operatorname{TV}\left(\Sigma_{v_{i},+}\right)$ is an open subset of $\mathrm{TV}(\Sigma)$. Using coordinates $x_{1}, \ldots, x_{n}$ on $\mathrm{TV}\left(\Sigma_{v_{i},+}\right)$ as in the proof of Lemma 3.1, we obtain an open subset of $\widetilde{T V}(\Sigma)$ described as a subset of $\operatorname{TV}\left(\Sigma_{v_{i},+}\right) \times \mathbb{P}^{1}$ given by the equation $u x_{1}=v \prod_{j \in J} f_{j}$. With this description, we define $\iota_{i}: T_{i} \rightarrow \tilde{U}_{\Sigma}$ by

$$
\iota_{i}:\left(x_{1}, \ldots, x_{n}\right) \mapsto\left(\left(\prod_{j \in J \backslash\{i\}} f_{j}, x_{1}\right),\left(f_{i} x_{1}, x_{2}, \ldots, x_{n}\right)\right)
$$

Note that $\iota_{i}$ contracts the locus $f_{i}=\prod_{j \in J \backslash\{i\}} f_{j}=0$ in $T_{i}$, so this is not an embedding unless the $Z_{j}$ are disjoint. In this coordinate chart, $\iota_{0}$ is given by

$$
\iota_{0}:\left(x_{1}, \ldots, x_{n}\right) \mapsto\left(\left(\prod_{j \in J} f_{j}, x_{1}\right),\left(x_{1}, \ldots, x_{n}\right)\right) .
$$

From this one sees that $\iota_{i} \circ \mu_{i}=\iota_{0}$ on $U_{0 i}$. In particular, the maps $\iota_{i}, 0 \leqslant i \leqslant n$ are compatible with the gluings $\varphi_{i j}$, and hence we obtain the desired map $\psi$.

In case (i), each $\iota_{i}, i \geqslant 1$, is an open immersion outside a set of codimension at least two, and as in Lemma 3.1, it is easy to see that the image misses a codimension $\geqslant 2$ set. In case (ii), each $\iota_{i}$ is an open immersion. Thus $\psi$ is a local isomorphism, and it is enough to show that $\psi$ is injective to see that it is an open immersion. Certainly $\psi$ is injective on each $T_{i}$. If $x \in T_{i}, y \in T_{j}$ have $\psi(x)=\psi(y)$, then $\iota_{i}(x)=\iota_{j}(y)$. Noting that $\varphi_{i j}=\iota_{j}^{-1} \circ \iota_{i}$ as rational maps, we see that $\varphi_{i j}$ is a local isomorphism at $x$ and $\varphi_{i j}(x)=y$. Thus $x \in U_{i j}$ and $x$ and $y$ are identified by the gluing maps, so they give the same point in $X$.

Next we understand the general setup for a mutation.

Given elements $v \in N, w \in M$ with $\langle w, v\rangle=0$, define the piecewise linear transformation

$$
T_{v, w}: N_{\mathbb{R}} \rightarrow N_{\mathbb{R}}, \quad n \mapsto n+[\langle n, w\rangle]_{-} v
$$

Note that this coincides with the tropicalization of $\mu_{(v, w)}$ in (1.2) as given in (1.4).

Now in the situation of this construction, let us impose one additional restriction on the starting data $v_{i}, w_{i}$, namely,

$$
\left\langle w_{i}, v_{j}\right\rangle=0 \Leftrightarrow\left\langle w_{j}, v_{i}\right\rangle=0
$$

Pick some index $k$ and let

$$
\Sigma_{+}=\Sigma \cup\left\{\mathbb{R}_{\leqslant 0} v_{k}\right\}
$$

and define $\Sigma_{-}$by applying $T_{-v_{k}, a_{k} w_{k}}$ to each ray of $\Sigma_{+}$. Let $D_{k,+} \subseteq \operatorname{TV}\left(\Sigma_{+}\right)$be the divisor corresponding to $\mathbb{R}_{\geqslant 0} v_{k}$ in $\Sigma_{+}$and let $D_{k,-} \subseteq \mathrm{TV}\left(\Sigma_{-}\right)$be the divisor corresponding to $\mathbb{R}_{\leqslant 0} v_{k}$ in $\Sigma_{-}$. For $j \neq k$, write $D_{j, \pm}$ for the divisor corresponding to $\mathbb{R}_{\geqslant 0} v_{j}$ in $\Sigma_{+}$or to $\mathbb{R}_{\geqslant 0} T_{-v_{k}, a_{k} w_{k}}\left(v_{j}\right)$ 


\section{Mark Gross, Paul Hacking and Sean Keel}

in $\Sigma_{-}$. Finally, we can set

$$
\begin{aligned}
Z_{j,+} & =\bar{V}\left(f_{j}\right) \cap D_{j,+} \\
Z_{j,-} & = \begin{cases}\bar{V}\left(f_{j}\right) \cap D_{j,-} & \text { if }\left\langle w_{k}, v_{j}\right\rangle \geqslant 0, \\
\bar{V}\left(\left(1+c_{j} c_{k}^{a_{k}\left\langle w_{j}, v_{k}\right\rangle} z^{w_{j}+a_{k}\left\langle w_{j}, v_{k}\right\rangle w_{k}}\right)^{a_{j}}\right) \cap D_{j,-} & \text { if }\left\langle w_{k}, v_{j}\right\rangle \leqslant 0 .\end{cases}
\end{aligned}
$$

Let $\widetilde{\mathrm{TV}}\left(\Sigma_{ \pm}\right)$be the blowups of $\operatorname{TV}\left(\Sigma_{ \pm}\right)$at this collection of subschemes.

Lemma 3.6. The map $\mu_{k}=\mu_{f_{k}}: T_{N} \rightarrow T_{N}$ defines a birational map

$$
\mu_{k}: \widetilde{\mathrm{TV}}\left(\Sigma_{+}\right) \rightarrow \widetilde{\mathrm{TV}}\left(\Sigma_{-}\right) \text {. }
$$

If $\operatorname{dim} \bar{V}\left(f_{k}\right) \cap Z_{j,+}<\operatorname{dim} Z_{j,+}$ whenever $\left\langle w_{k}, v_{j}\right\rangle=0$, then this extension is an isomorphism outside sets of codimension at least two.

Proof. We first analyze the map $\mu_{k}$ before blowing up the hypersurfaces $Z_{j,+}, j \neq k$. So abusing notation, assume that $\widetilde{\mathrm{TV}}\left(\Sigma_{ \pm}\right)$is just obtained by blowing up $Z_{k, \pm}$. Off of a closed subset of codimension two, we can cover $\widetilde{\mathrm{TV}}\left(\Sigma_{+}\right)$with open sets, one isomorphic to $\tilde{\mathbb{P}}_{+}$with $v=v_{k}$, and the remaining ones of the form $U_{\rho} \backslash \bar{V}\left(f_{k}\right)$. Here $\rho$ ranges over dimension one cones of $\Sigma_{+}$ not equal to $\mathbb{R}_{\geqslant 0} v_{k}$ or $\mathbb{R}_{\leqslant 0} v_{k}$, and $U_{\rho}$ denotes the standard affine toric open subset of $\operatorname{TV}\left(\Sigma_{+}\right)$ corresponding to $\rho$. Denoting $D_{\rho} \subseteq U_{\rho}$ the toric divisor, note that $D_{\rho} \cap \bar{V}\left(f_{k}\right)=\emptyset$ if $w_{k}$ is non-zero on $\rho$, as then either $z^{w_{k}}$ or $z^{-w_{k}}$ vanishes on $D_{\rho}$ and $\bar{V}\left(1+z^{w_{k}}\right)=\bar{V}\left(1+z^{-w_{k}}\right)$. Thus we only fail to cover codimension two subsets of the form $D_{\rho} \cap \bar{V}\left(f_{k}\right)$ such that $w_{k}$ is zero on $\rho$. So for the purposes of describing the extension of $\mu_{k}$ up to codimension two, it will be sufficient to restrict to the open subset $U$ of $\widetilde{\mathrm{TV}}\left(\Sigma_{+}\right)$covered by these open sets.

By Lemma 3.2, $\mu_{k}$ gives a well-defined morphism on the open subset isomorphic to $\tilde{\mathbb{P}}_{+}$, so we need to check that $\mu_{k}$ defines a morphism on each of the remaining sets. If $\left\langle w_{k}, \rho\right\rangle \geqslant 0$, then for any $m \in \rho^{\vee} \cap M=\left(T_{-v_{k}, a_{k} w_{k}}(\rho)\right)^{\vee} \cap M, \mu_{k}^{*}$ acts by

$$
z^{m} \mapsto z^{m} f_{k}^{-\left\langle m, v_{k}\right\rangle},
$$

taking a regular function to a regular function on $U_{\rho} \backslash \bar{V}\left(f_{k}\right)$. If $\left\langle w_{k}, \rho\right\rangle<0$, then if $m \in$ $\left(T_{-v_{k}, a_{k} w_{k}}(\rho)\right)^{\vee} \cap M$, we see that $\mu_{k}$ acts by

$$
z^{m} \mapsto z^{m}\left(1+c_{k} z^{w_{k}}\right)^{-a_{k}\left\langle m, v_{k}\right\rangle}=z^{m-a_{k}\left\langle m, v_{k}\right\rangle w_{k}}\left(c_{k}+z^{-w_{k}}\right)^{-a_{k}\left\langle m, v_{k}\right\rangle} .
$$

But $m-a_{k}\left\langle m, v_{k}\right\rangle w_{k} \in \rho^{\vee}$ by definition of $T_{-v_{k}, a_{k} w_{k}}$, so this is again a regular function on $U_{\rho} \backslash \bar{V}\left(f_{k}\right)$. This shows that $\mu_{k}$ is a morphism on $U$; to show that it is an isomorphism onto its image, we repeat the same process for $\mu_{k}^{-1}$.

To prove the result after blowing up the hypersurfaces $Z_{j, \pm}$, first note that if $\left\langle w_{k}, v_{j}\right\rangle \neq 0$, then $Z_{j,+} \subseteq U$, and we need to show that $\mu_{k}\left(Z_{j,+}\right)=Z_{j,-}$. This can be checked in cases. If $\left\langle w_{k}, v_{j}\right\rangle \geqslant 0$, then $Z_{j,-}$ is defined by the equation $f_{j}$ on $D_{j}$. Now if $\left\langle w_{k}, v_{j}\right\rangle>0$, we have $\left.f_{k}\right|_{D_{j}}=1$, so that $\left.\mu_{k}^{*}\left(f_{j}\right)\right|_{D_{j}}=\left.f_{j}\right|_{D_{j}}$. If $\left\langle w_{k}, v_{j}\right\rangle=0$, then $\left\langle w_{j}, v_{k}\right\rangle=0$ by assumption (3.1), so that $\mu_{k}^{*} z^{w_{j}}=z^{w_{j}}$, so again $\mu_{k}^{*}\left(f_{j}\right)=f_{j}$. If $\left\langle w_{k}, v_{j}\right\rangle<0$, then noting the definition of $Z_{j,-}$ in this case,

$$
\begin{aligned}
\mu_{k}^{*}\left(\left(1+c_{j} c_{k}^{a_{k}\left\langle w_{j}, v_{k}\right\rangle} z^{w_{j}+a_{k}\left\langle w_{j}, v_{k}\right\rangle w_{k}}\right)^{a_{j}}\right) & =\left(1+c_{j} c_{k}^{a_{k}\left\langle w_{j}, v_{k}\right\rangle} z^{w_{j}+a_{k}\left\langle w_{j}, v_{k}\right\rangle w_{k}}\left(1+c_{k} z^{w_{k}}\right)^{-a_{k}\left\langle w_{j}, v_{k}\right\rangle}\right)^{a_{j}} \\
& =\left(1+c_{j} c_{k}^{a_{k}\left\langle w_{j}, v_{k}\right\rangle} z^{w_{j}}\left(c_{k}+z^{-w_{k}}\right)^{-a_{k}\left\langle w_{j}, v_{k}\right\rangle}\right)^{a_{j}} .
\end{aligned}
$$

However, $z^{-w_{k}}$ vanishes identically on $D_{j}$ in this case, so when restricting to $D_{j}$, this coincides with $f_{j}$. This shows that $\mu_{k}$ extends to a regular map after blowing up $U$ along the $Z_{j, \pm}$ for those $j$ with $\left\langle w_{k}, v_{j}\right\rangle \neq 0$. 


\section{BiRATIONAL GEOMETRY OF CLUSTER ALGEBRAS}

Finally, if $\left\langle w_{k}, v_{j}\right\rangle=0$, then we do not necessarily have $Z_{j,+} \subseteq U$, and if $\bar{V}\left(f_{k}\right)$ contains an irreducible component of $Z_{j,+}$, the map $\mu_{k}$ need not extend as an isomorphism across the exceptional divisor of the blowup of $Z_{j,+}$. Hence we need to use the stated hypothesis, which implies that $Z_{j,+} \backslash U$ is of codimension at least three. Since $\mu_{k}^{*}\left(f_{j}\right)=f_{j}$ when $\left\langle w_{k}, v_{j}\right\rangle=0$, it then follows that $\mu_{k}$ extends to an isomorphism outside a set of codimension at least two in $\widetilde{\mathrm{TV}}\left(\Sigma_{+}\right)$.

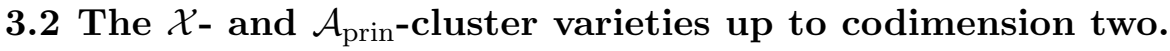

Since the ring of functions on a non-singular variety is determined outside a set of codimension

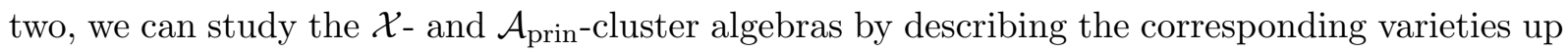
to codimension two.

Suppose given fixed data as in $\S 2$. Let $\mathbf{s}$ be a seed. Consider the fans

$$
\begin{aligned}
& \Sigma_{\mathbf{s}, \mathcal{A}}:=\{0\} \cup\left\{\mathbb{R}_{\geqslant 0} d_{i} e_{i} \mid i \in I_{\mathrm{uf}}\right\}, \\
& \Sigma_{\mathbf{s}, \mathcal{X}}:=\{0\} \cup\left\{-\mathbb{R}_{\geqslant 0} d_{i} v_{i} \mid i \in I_{\mathrm{uf}}\right\}
\end{aligned}
$$

in $N^{\circ}$ and $M$, respectively. These define toric varieties $\mathrm{TV}_{\mathbf{s}, \mathcal{A}}$ and $\mathrm{TV}_{\mathbf{s}, \mathcal{X}}$ respectively. We remark that the minus signs in the definition of $\Sigma_{\mathbf{s}, \mathcal{X}}$ are forced on us by (2.10).

Each one-dimensional ray in one of these fans corresponds to a toric divisor, which we write as $D_{i}$ for $i \in I_{\text {uf }}$ (not distinguishing the $\mathcal{X}$ and $\mathcal{A}$ cases). For $i \in I_{\text {uf }}$, we can define closed subschemes

$$
\begin{aligned}
& Z_{\mathcal{A}, i}:=D_{i} \cap \bar{V}\left(1+z^{v_{i}}\right) \subseteq \mathrm{TV}_{\mathbf{s}, \mathcal{A}}, \\
& Z_{\mathcal{X}, i}:=D_{i} \cap \bar{V}\left(\left(1+z^{e_{i}}\right)^{\text {ind } d_{i} v_{i}}\right) \subseteq \mathrm{TV}_{\mathbf{s}, \mathcal{X}},
\end{aligned}
$$

where ind $d_{i} v_{i}$ denotes the greatest degree of divisibility of $d_{i} v_{i}$ in $M$. Let $\left(\widetilde{\mathrm{TV}}_{\mathbf{s}, \mathcal{A}}, D\right)$ and $\left(\widetilde{\mathrm{TV}}_{\mathbf{s}, \mathcal{X}}, D\right)$ be the pairs consisting of the blowups of $\mathrm{TV}_{\mathbf{s}, \mathcal{A}}$ and $\mathrm{TV}_{\mathbf{s}, \mathcal{X}}$ along the closed subschemes $Z_{\mathcal{A}, i}$ and $Z_{\mathcal{X}, i}$, respectively, with $D$ the proper transform of the toric boundaries.

We note that in the $\mathcal{A}$ case the divisors $D_{i}$ are distinct and hence the centers of the blowups are disjoint. In the $\mathcal{X}$ case, however, $v_{i}$ and $v_{i^{\prime}}$ might be positively proportional to each other, so that $D_{i}=D_{i^{\prime}}$. Then the two centers $Z_{\mathcal{X}, i}, Z_{\mathcal{X}, i^{\prime}}$ may intersect. However, it is easy to see that this intersection occurs in higher codimension, that is, $\operatorname{dim} Z_{\mathcal{X}, i} \cap Z_{\mathcal{X}, i^{\prime}}<\operatorname{dim} Z_{\mathcal{X}, i}$. Thus in the $\mathcal{X}$ case, we are in the situation of Lemma 3.5(i) and in the $\mathcal{A}$ case, we are in the situation of Lemma 3.5(ii).

Finally, we define $U_{\mathbf{s}, \mathcal{A}}:=\widetilde{\mathrm{TV}}_{\mathbf{s}, \mathcal{A}} \backslash D$ and $U_{\mathbf{s}, \mathcal{X}}:=\widetilde{\mathrm{TV}}_{\mathbf{s}, \mathcal{X}} \backslash D$. Clearly, these varieties contain the seed tori $\mathcal{A}_{\mathbf{s}}$ and $\mathcal{X}_{s}$; hence, given vertices $w, w^{\prime} \in \mathfrak{T}$, we obtain a birational map $\mu_{w, w^{\prime}}$ of seed tori inducing birational maps

$$
\mu_{w, w^{\prime}}: U_{\mathbf{s}_{w}, \mathcal{A}} \rightarrow U_{\mathbf{s}_{w^{\prime}}, \mathcal{A}}, \quad \mu_{w, w^{\prime}}: U_{\mathbf{s}_{w}, \mathcal{X}} \rightarrow U_{\mathbf{s}_{w^{\prime}}, \mathcal{X}}
$$

Since $\mathcal{A}_{\text {prin }}$ is defined to be a special case of the construction of the $\mathcal{A}$ cluster variety, we also obtain in the same way birational maps

$$
\mu_{w, w^{\prime}}: U_{\mathbf{s}_{w}, \mathcal{A}_{\text {prin }}} \rightarrow U_{\mathbf{s}_{w^{\prime}}, \mathcal{A}_{\text {prin }}} .
$$

In this case the projection $\widetilde{N}^{\circ} \rightarrow M$ projects all rays of $\Sigma_{\mathbf{s}, \mathcal{A}_{\text {prin }}}$ to 0 , so we obtain a morphism $\mathrm{TV}_{\mathbf{s}, \mathcal{A}_{\text {prin }}} \rightarrow T_{M}$. The fibres of this map are (non-canonically) isomorphic to $\mathrm{TV}_{\mathbf{s}, \mathcal{A}}$. After blowing up the centers $Z_{\mathcal{A}_{\text {prin }}, i}$, we get morphisms $\pi: U_{\mathrm{s}, \mathcal{A}_{\text {prin }}} \rightarrow T_{M}$ which commute with the mutations $\mu_{w, w^{\prime}}$. Write a fibre of $\pi$ over $t \in T_{M}$ as $U_{\mathbf{s}, \mathcal{A}_{t}}$. We then obtain birational maps on fibres of $\pi$ 


\section{Mark Gross, Paul Hacking and Sean Keel}

over $t$,

$$
\mu_{w, w^{\prime}}: U_{\mathbf{s}_{w}, \mathcal{A}_{t}} \rightarrow U_{\mathbf{s}_{w^{\prime}}, \mathcal{A}_{t}} .
$$

We recall the following definition from [BFZ05].

Definition 3.7. A seed $\mathbf{s}$ is coprime if, writing (2.8) as $A_{k} \cdot \mu_{k}^{*} A_{k}^{\prime}=P_{k}$, the $P_{k}, k \in I_{\mathrm{uf}}$, are pairwise coprime. We say that a seed $\mathbf{s}$ is totally coprime if all seeds obtained by repeated mutations of $\mathbf{s}$ are coprime.

We then have the following result.

Lemma 3.8. Let $U_{\mathbf{s}, \mathcal{A}}^{\prime} \subset \mathcal{A}$ (respectively, $U_{\mathbf{s}, \mathcal{X}}^{\prime} \subset \mathcal{X}$ ) be the union of the tori $\mathcal{A}_{\mathbf{s}}$ (respectively, $\left.\mathcal{X}_{\mathbf{s}}\right)$ and $\mathcal{A}_{\mu_{i}(\mathbf{s})}$ (respectively, $\left.\mathcal{X}_{\mu_{i}(\mathbf{s})}\right), i \in I_{\mathrm{uf}}$.

(i) For $k \in I_{\text {uf }}$, with $w^{\prime}=\mu_{k}(w)$, the maps

$$
\mu_{w, w^{\prime}}: U_{\mathbf{s}_{w}, \mathcal{X}} \rightarrow U_{\mathbf{s}_{w^{\prime}}, \mathcal{X}}, \quad \mu_{w, w^{\prime}}: U_{\mathbf{s}_{w}, \mathcal{A}_{\text {prin }}} \rightarrow U_{\mathbf{s}_{w^{\prime}}, \mathcal{A}_{\text {prin }}}
$$

are isomorphisms outside codimension two.

(ii) The map $\mu_{w, w^{\prime}}: U_{\mathbf{s}_{w}, \mathcal{A}} \rightarrow U_{\mathbf{s}_{w^{\prime}}, \mathcal{A}}$ is an isomorphism outside codimension two if the seed $\mathbf{s}_{w}$ is coprime.

(iii) The map $\mu_{w, w^{\prime}}: U_{\mathbf{s}_{w}, \mathcal{A}_{t}} \rightarrow U_{\mathbf{s}_{w^{\prime}}, \mathcal{A}_{t}}$ is an isomorphism outside codimension two for $t \in T_{M}$ general (that is, $t$ contained in some non-empty Zariski open subset).

(iv) The map $U_{\mathbf{s}, \mathcal{A}}^{\prime} \rightarrow U_{\mathbf{s}, \mathcal{A}}$ is an open immersion with image an open subset whose complement has codimension at least two.

(v) The map $U_{\mathbf{s}, \mathcal{X}}^{\prime} \rightarrow U_{\mathbf{s}, \mathcal{X}}$ is an isomorphism outside a set of codimension two.

Proof. These are all special cases of construction 3.4. For cases (i) and (ii), in the $\mathcal{X}$ (respectively, $\left.\mathcal{A}_{\text {prin }}, \mathcal{A}\right)$ case, we take the vectors $v_{i}$ to be $-d_{i} v_{i} / \operatorname{ind}\left(d_{i} v_{i}\right) \in M$ (respectively, $\left(d_{i} e_{i}, 0\right) \in \tilde{N}^{\circ}$, $d_{i} e_{i} \in N^{\circ}$ ) for $i \in I_{\mathrm{uf}}$, the vectors $w_{i}$ to be $e_{i} \in N$ (respectively, $\left(v_{i}, e_{i}\right) \in \widetilde{M}^{\circ}, v_{i} \in M^{\circ}$ ). In all these cases, the constants $c_{i}$ are taken to be 1 . The integers $a_{i}$ are taken to be $a_{i}=\operatorname{ind}\left(d_{i} v_{i}\right)$ (respectively, $a_{i}=1$ ). In all three cases, the cluster mutation $\mu_{k}$ coincides with the $\mu_{k}$ as defined in Construction 3.4. In the notation of Lemma 3.6, taking $\Sigma_{+}=\Sigma_{\mathbf{s}_{w}, \mathcal{X}}$ (respectively, $\Sigma_{\mathbf{s}_{w}, \mathcal{A}_{\text {prin }}}$, $\Sigma_{\mathbf{S}_{w}, \mathcal{A}}$ ), we observe that $T_{d_{k} v_{k}, e_{k}}$ (respectively, $T_{\left(-d_{k} e_{k}, 0\right),\left(v_{k}, e_{k}\right)}, T_{-d_{k} e_{k}, v_{k}}$ ) applied to the rays of $\Sigma_{+}$gives $\Sigma_{-}:=\Sigma_{\mathbf{s}_{w^{\prime}}, \mathcal{X}}$, (respectively, $\Sigma_{\mathbf{s}_{w^{\prime}}, \mathcal{A}_{\text {prin }}}, \Sigma_{\mathbf{s}_{w^{\prime}}, \mathcal{A}}$ ) as follows immediately from (2.3) and Remark 2.3.

We now only need to check the hypothesis of Lemma 3.6 to see that $\mu_{w, w^{\prime}}$ is an isomorphism outside codimension two subsets. In the $\mathcal{X}$ case, $f_{k}=1+z^{e_{k}}$, and from this the condition is easily checked. In the $\mathcal{A}$ case, $f_{k}=1+z^{v_{k}}$, which coincides with $P_{k}$ up to a monomial factor. The hypothesis then follows from the coprime condition, and the principal coefficient case is automatically coprime as the $\left(v_{k}, e_{k}\right), k \in I_{\mathrm{uf}}$, are linearly independent.

The $\mathcal{A}_{t}$ case (iii) is similar to the $\mathcal{A}$ case, except that now $f_{k}=1+z^{e_{k}}(t) \cdot z^{v_{k}}$, so we take $c_{k}=z^{e_{k}}(t)$. If $t$ is chosen generally, then the hypothesis of Lemma 3.6 continues to hold.

Case (v) follows from part (i) of Lemma 3.5. Case (iv) follows from part (ii) of Lemma 3.5.

The main result in this section is then as follows. 


\section{BirATIONAL GEOMETRY OF CLUSTER ALGEBRAS}

THEOREM 3.9. Let $w, w^{\prime}$ be vertices in $\mathfrak{T}$.

(i) The induced birational maps

$$
\begin{aligned}
& \stackrel{\mu_{w, w^{\prime}}}{U_{\mathbf{s}_{w}, \mathcal{X}} \stackrel{---\rightarrow}{ } U_{\mathbf{s}_{w^{\prime}}, \mathcal{X} \rightarrow-\rightarrow}} \mathcal{X}^{\mathrm{ft}} \\
& U_{\mathbf{s}_{w}, \mathcal{A}_{\text {prin }}} \stackrel{\mu_{w, w^{\prime}}}{----\rightarrow} U_{\mathbf{s}_{w^{\prime}}, \mathcal{A}_{\text {prin }}} \rightarrow-\rightarrow \mathcal{A}_{\text {prin }}^{\mathrm{ft}}
\end{aligned}
$$

are isomorphisms outside a codimension two set. (See Remark 2.6 for $\mathcal{X}^{\mathrm{ft}}, \mathcal{A}^{\mathrm{ft}}$. We use a finite subtree of $\mathfrak{T}$ containing both $w$ and $w^{\prime}$.)

(ii) If the initial seed is totally coprime, then

$$
U_{\mathbf{s}_{w}, \mathcal{A}} \stackrel{\mu_{w, w^{\prime}}}{----\rightarrow \rightarrow} U_{\mathbf{s}_{w^{\prime}}, \mathcal{A}} \rightarrow \mathcal{A}^{\mathrm{ft}}
$$

is an isomorphism outside a codimension two set.

(iii) If $t \in T_{M}$ is very general (outside a countable union of proper closed subsets), then

$$
U_{\mathbf{s}_{w}, \mathcal{A}_{t}} \stackrel{\mu_{w, w^{\prime}}}{----\rightarrow} U_{\mathbf{s}_{w^{\prime}}, \mathcal{A}_{t}} \rightarrow \mathcal{A}_{t}^{\mathrm{ft}}
$$

is an isomorphism outside a codimension two set.

In particular, as all schemes involved are $S_{2}$, these maps induce isomorphisms on rings of regular functions.

Proof. That the maps $\mu_{w, w^{\prime}}$ are isomorphisms outside codimension two sets follows from Lemma 3.8. For the remaining statements in (i-iii), consider just the $\mathcal{X}$ case, as the other cases are identical. By Lemma 3.5, each of the $U_{\mathbf{s}, \mathcal{X}}$ is isomorphic, outside a codimension two set, to the gluing of the seed torus $\mathcal{X}_{\mathbf{s}}$ to its adjacent seed tori $\mathcal{X}_{\mu_{k}(\mathbf{s})}, k \in I_{\mathrm{uf}}$. This gives a birational map $U_{\mathbf{s}, \mathcal{X}} \rightarrow \mathcal{X}^{\mathrm{ft}} \subseteq \mathcal{X}$. (Here we use any choice of regular subtree of $\mathfrak{T}$ containing the vertex corresponding to $\mathbf{s}$ and its adjacent vertices. The subtree is taken to be finite but as large as we would like.) Since $\mathcal{X}^{\mathrm{ft}}$ is covered, up to codimension two subsets, by some finite collection $\left\{U_{\mathbf{s}_{w}, \mathcal{X}}\right\}$, we see that each $U_{\mathbf{s}_{w}, \mathcal{X}}$ is isomorphic to $\mathcal{X}^{\mathrm{ft}}$ outside a codimension two subset. We need to use $\mathcal{X}^{\mathrm{ft}}$ rather than $\mathcal{X}$, for if $\mathcal{X}$ is not Noetherian, the subset of $\mathcal{X}$ we fail to cover need not be closed.

Remark 3.10. More generally than the principal coefficient case, the totally coprime hypothesis also holds if the matrix $\left(\epsilon_{i j}\right)_{i \in I_{\mathrm{uf}}, 1 \leqslant j \leqslant n}$ has full rank; see [BFZ05, Proposition 1.8]. Of course, this holds in particular for the principal coefficient case.

We immediately obtain from this a geometric explanation for the well-known Laurent phenomenon.

Corollary 3.11 (The Laurent phenomenon). For a seed $\mathbf{s}$, let $q \in M^{\circ}$ (respectively, $q \in N$ ) have non-negative pairing with each $e_{i}$ (respectively, each $-v_{i}$ ) for $i \in I_{\mathrm{uf}}$. Equivalently, $z^{q}$ is a monomial which is a regular function on the toric variety $\mathrm{TV}_{\mathbf{s}, \mathcal{A}}$ (respectively, $\mathrm{TV}_{\mathbf{s}, \mathcal{X}}$ ). Then $z^{q}$ is a Laurent polynomial on every seed torus, that is, $z^{q} \in H^{0}\left(\mathcal{A}, \mathcal{O}_{\mathcal{A}}\right)$ (respectively, $\left.z^{q} \in H^{0}\left(\mathcal{X}, \mathcal{O}_{\mathcal{X}}\right)\right)$.

Proof. By assumption, $z^{q}$ is a regular function on $\mathrm{TV}_{\mathbf{s}, \mathcal{A}}$ (or $\mathrm{TV}_{\mathbf{s}, \mathcal{X}}$ ), and hence pulls back and restricts to a regular function on $U_{\mathbf{s}, \mathcal{A}}$ (respectively, $U_{\mathbf{s}, \mathcal{X}}$ ). In the $\mathcal{X}$ case, the result then follows from Theorem 3.9, since then $z^{q}$ also defines a regular function on $\mathcal{X}^{\mathrm{ft}}$ for any choice of subtree of $\mathfrak{T}$, and hence also defines a regular function on $\mathcal{X}$. 


\section{Mark Gross, Paul Hacking and Sean Keel}

The $\mathcal{A}$ case then follows from the $\mathcal{A}_{\text {prin }}$ case, since the mutation formula (2.6) for $\mathcal{A}$ is obtained from that for $\mathcal{A}_{\text {prin }}$ by setting $z^{\left(0, e_{i}\right)}=1$ for $\left(e_{1}, \ldots, e_{n}\right)$ the initial seed.

Remark 3.12. Note that in the $\mathcal{A}$ case, with no frozen variables, (that is, $I_{\mathrm{uf}}=I$ ) the condition on $q$ is exactly that $q$ is in the non-negative span of the $e_{i}^{*}$, that is, that $z^{q}$ is a monomial, with non-negative exponents, in the cluster variables of the seed. In particular, this applies to any cluster variable, in which case the statement gives the usual Laurent phenomenon. From this point of view the difference between $\mathcal{A}$ and $\mathcal{X}$ is that the fan $\Sigma_{\mathbf{s}, \mathcal{A}}$ always looks the same (it is the union of coordinate rays), and, in particular, $\mathrm{TV}_{\mathbf{s}, \mathcal{A}}$ has many global functions (this is a toric open subset of $\mathbb{A}^{n}$ ), while $\Sigma_{\mathbf{s}, \mathcal{X}}$ can be any arbitrary collection of rays, and $\mathrm{TV}_{\mathbf{s}, \mathcal{X}}$ has non-constant global functions if and only if all these rays lie in a common half space.

Remark 3.13. By [BFZ05, Definition 1.1], the algebra $H^{0}\left(U_{\mathbf{s}, \mathcal{A}}^{\prime}, \mathcal{O}_{U_{\mathbf{s}, \mathcal{A}}^{\prime}}\right)=H^{0}\left(U_{\mathbf{s}, \mathcal{A}}, \mathcal{O}_{U_{\mathbf{s}, \mathcal{A}}}\right)$ (see part (4) of Lemma 3.8) is the upper bound. In an earlier version of this paper we claimed Theorem 3.9 for $\mathcal{A}$ (without any coprimality assumption), which would, in particular, imply that the upper bound is equal to the upper cluster algebra. But Greg Muller set us straight, by giving us an example where the upper cluster algebra is strictly smaller than the upper bound.

We learned the following theorem, and its proof, from M. Shapiro.

Theorem 3.14. The canonical map $\iota: \mathcal{A} \rightarrow$ Spec $A^{\text {up }}$ is an open immersion, where $A^{\text {up }}=$ $\Gamma\left(\mathcal{A}, \mathcal{O}_{\mathcal{A}}\right)$ is the upper cluster algebra. In particular, $\mathcal{A}$ is separated.

Proof. The variety $\mathcal{A}$ is covered by open sets of the form $\mathcal{A}_{\mathbf{s}}$, for various seeds s. First, note that the induced map $\iota_{\mathbf{s}}: \mathcal{A}_{\mathbf{s}} \rightarrow \operatorname{Spec} A^{\text {up }}$ is an open immersion. Indeed, this map is induced by the inclusion $\iota_{\mathbf{s}}^{*}: A^{\text {up }} \subseteq \mathbb{k}\left[A_{1}^{ \pm 1}, \ldots, A_{n}^{ \pm 1}\right]=: B$, where $A_{1}, \ldots, A_{n}$ are the cluster coordinates on $\mathcal{A}_{\mathbf{s}}$. One checks that this is a local isomorphism: given $\left(a_{1}, \ldots, a_{n}\right) \in \mathcal{A}_{\mathbf{s}}, a_{1}, \ldots, a_{n} \neq 0$, the corresponding maximal ideal is $\mathfrak{m}=\left\langle A_{1}-a_{1}, \ldots, A_{n}-a_{n}\right\rangle \subseteq B$. By the Laurent phenomenon, $A_{1}, \ldots, A_{n} \in A^{\text {up }}$, and thus $A_{1}, \ldots, A_{n}$ are invertible in the localization $A_{\left(\iota_{\mathbf{s}}^{*}\right)^{-1}(\mathfrak{m})^{\text {up }}}$. Thus $A_{\left(\iota_{\mathbf{s}}^{*}\right)^{-1}(\mathfrak{m})}^{\text {up }} \cong B_{\mathfrak{m}}$, and $\iota_{\mathbf{s}}$ is a local isomorphism. Hence by [Gro60, I, 8.2.8], $\iota_{\mathbf{s}}$ is an open immersion.

To show that $\iota$ itself is now an open immersion, it is sufficient to show it is one-to-one since it is a local isomorphism. Let $x \in \mathcal{A}_{\mathbf{s}}, y \in \mathcal{A}_{\mathbf{s}^{\prime}}$ be such that $\iota(x)=\iota(y)$. Let $A_{1}, \ldots, A_{n}$ be the cluster coordinates on $\mathcal{A}_{\mathbf{s}}$. Again by the Laurent phenomenon, there is an inclusion $\mathbb{k}\left[A_{1}, \ldots, A_{n}\right] \subseteq A^{\text {up }}$, hence a map $\psi: \operatorname{Spec} A^{\text {up }} \rightarrow \mathbb{A}^{n}$. The composition $\psi \circ \iota_{\mathbf{s}}$ is the obvious inclusion and $\left(\psi \circ \iota_{\mathbf{s}}\right)^{-1} \circ\left(\psi \circ \iota_{\mathbf{s}^{\prime}}\right)$ agrees, as a rational map, with $\mu_{w^{\prime}, w}$, where $w^{\prime}, w$ are the vertices of $\mathfrak{T}$ corresponding to the seeds $\mathbf{s}^{\prime}, \mathbf{s}$. Thus the map $\mu_{w^{\prime}, w}$ is defined at $y$, since $\psi \circ \iota_{\mathbf{s}^{\prime}}$ is defined at $y$ and $\left(\psi \circ \iota_{\mathbf{S}}\right)^{-1}$ is defined at $\psi\left(\iota_{s^{\prime}}(y)\right)=\psi\left(\iota_{\mathbf{S}}(x)\right)$. Furthermore, $\mu_{w^{\prime}, w}$ is then a local isomorphism at $y$ as it agrees with $\iota_{\mathbf{s}}^{-1} \circ \iota_{\mathbf{s}^{\prime}}$ at $y$, and $\iota_{\mathbf{s}}$ and $\iota_{\mathbf{s}^{\prime}}$ are local isomorphisms at $x$ and $y$, respectively. So the gluing map defining $\mathcal{A}$ identifies $x$ and $y$, and $\iota$ is injective.

\section{The $\mathcal{A}_{t}$ and $\mathcal{A}_{\text {prin }}$ cluster varieties as torsors}

We fix in this section fixed data and a seed $\mathbf{s}$ as usual. We shall assume that there are no frozen variables, that is, $I_{\mathrm{uf}}=I, N_{\mathrm{uf}}=N$, and furthermore that the matrix $\epsilon$ has no zero row (or equivalently no zero column). Note that if $\epsilon$ does have a zero row, the same is true for all mutations, so this condition is mutation independent. We then obtain the $\mathcal{X}, \mathcal{A}, \mathcal{A}_{\text {prin }}$ and $\mathcal{A}_{t}$ varieties. 


\section{Birational GEOMETRY OF CLUSTER ALGEBRAS}

Denote by $X$ the open subset of $\mathcal{X}$ obtained by gluing together the seed tori $\mathcal{X}_{\mathbf{s}}$ and $\mathcal{X}_{\mu_{k}(\mathbf{s})}$, $1 \leqslant k \leqslant n$. This still comes with a map $\lambda: X \rightarrow T_{K^{*}}$ as in construction 2.13, and we write $X_{\phi}$ for the fibre over $\phi \in T_{K^{*}}$.

We first compute the Picard group of $X$ and $X_{\phi}$.

Theorem 4.1. For $\phi \in T_{K^{*}}, \operatorname{Pic}(X) \cong \operatorname{Pic}\left(X_{\phi}\right) \cong \operatorname{coker}\left(p^{*}: N \rightarrow M^{\circ}\right)$.

Proof. We first need to describe precisely how $X$ and $X_{\phi}$ are glued together out of tori. Let $U_{0}=\mathcal{X}_{\mathbf{s}}, U_{i}=\mathcal{X}_{\mu_{i}(\mathbf{s})}, 1 \leqslant i \leqslant n$. We have birational gluing maps $\varphi_{i j}: U_{i} \rightarrow U_{j}$ given by $\varphi_{0 j}=\mu_{j}, \varphi_{i j}=\mu_{j} \circ \mu_{i}^{-1}$. These glue over sets $U_{i j}$ as in Proposition 2.4. Note that

$$
U_{0 j}=\mathcal{X}_{\mathbf{s}} \backslash V\left(1+z^{e_{j}}\right),
$$

by (2.5) and the fact that no row or column of $\epsilon$ is zero. The same description applies to $U_{j 0}$. On the other hand, noting that

$$
\left(\mu_{j} \circ \mu_{i}^{-1}\right)^{*}\left(z^{n}\right)=z^{n}\left(1+z^{e_{j}}\left(1+z^{e_{i}}\right)^{\left[e_{j}, e_{i}\right]}\right)^{\left[-n, e_{j}\right]}\left(1+z^{e_{i}}\right)^{\left[n, e_{i}\right]},
$$

one sees that if we set

$$
h_{i j}= \begin{cases}1+z^{e_{j}}\left(1+z^{e_{i}}\right)^{\left[e_{j}, e_{i}\right]} & \text { for }\left[e_{j}, e_{i}\right] \geqslant 0 \\ \left(1+z^{e_{i}}\right)^{-\left[e_{j}, e_{i}\right]}+z^{e_{j}} & \text { for }\left[e_{j}, e_{i}\right] \leqslant 0\end{cases}
$$

then

$$
U_{i j}=\mathcal{X}_{\mu_{i}(\mathbf{s})} \backslash\left(V\left(1+z^{e_{i}}\right) \cup V\left(h_{i j}\right)\right) .
$$

Now the $U_{i j}$ also map to $T_{K^{*}}$, with fibres $U_{i j, \phi}$ over $\phi$, so that $X_{\phi}$ is obtained by gluing the sets $U_{i, \phi}$ (the fibres of $U_{i} \rightarrow T_{K^{*}}$ over $\phi$ ) via the restriction of the $\varphi_{i j}$ to $U_{i j, \phi}$. Choose a splitting $N=K \oplus N^{\prime}$. A regular function on a fibre of $\mathcal{X}_{s} \rightarrow T_{K^{*}}$ is a linear combination of restrictions of monomials $z^{n^{\prime}}, n^{\prime} \in N^{\prime}$, to the fibre.

In particular, we have

$$
\begin{aligned}
\Gamma\left(U_{0 i}, \mathcal{O}_{U_{0 i}}^{\times}\right) & =\left\{c z^{n}\left(1+z^{e_{i}}\right)^{-a} \mid c \in \mathbb{k}^{\times}, n \in N, a \in \mathbb{Z}\right\}, \\
\Gamma\left(U_{i j}, \mathcal{O}_{U_{i j}}^{\times}\right) & =\left\{c z^{n}\left(1+z^{e_{i}}\right)^{-a} h_{i j}^{-b} \mid c \in \mathbb{k}^{\times}, n \in N, a, b \in \mathbb{Z}\right\}, \\
\Gamma\left(U_{0 i, \phi}, \mathcal{O}_{U_{0 i, \phi}}^{\times}\right) & =\left\{c z^{n}\left(1+z^{e_{i}}\right)^{-a} \mid c \in \mathbb{k}^{\times}, n \in N^{\prime}, a \in \mathbb{Z}\right\}, \\
\Gamma\left(U_{i j, \phi}, \mathcal{O}_{U_{i j, \phi}}^{\times}\right) & =\left\{c z^{n}\left(1+z^{e_{i}}\right)^{-a} h_{i j}^{-b} \mid c \in \mathbb{k}^{\times}, n \in N^{\prime}, a, b \in \mathbb{Z}\right\},
\end{aligned}
$$

noting that as $e_{i} \notin K$ for any $i$ by our assumption on $\epsilon, 1+z^{e_{i}}$ has some zeroes on $U_{i j, \phi}$. We will now compute $\operatorname{Pic}\left(X_{\phi}\right)$, the argument for $\operatorname{Pic}(X)$ being identical except that $N^{\prime}$ is replaced by $N$ below. We compute $\operatorname{Pic}\left(X_{\phi}\right)=H^{1}\left(X_{\phi}, \mathcal{O}_{X_{\phi}}^{\times}\right)$using the Cech cover $\left\{U_{i, \phi} \mid 0 \leqslant i \leqslant n\right\}$ with $U_{i, \phi} \cap U_{j, \phi}$ identified with $U_{i j, \phi}$ for $i<j$. Indeed, this cover calculates $\operatorname{Pic}\left(X_{\phi}\right)$ because $\operatorname{Pic}\left(U_{i, \phi}\right)=$ $\operatorname{Pic}\left(U_{i j, \phi}\right)=0$ for all $i$ and $j$. Thus a Čech 1-cochain consists of elements $g_{i j} \in \Gamma\left(U_{i j, \phi}, \mathcal{O}_{U_{i j, \phi}}^{\times}\right)$for each $i<j$. In particular, if $\left(g_{i j}\right)$ is a 1-cocycle, necessarily $g_{i j}=\left(\mu_{i}^{-1}\right)^{*}\left(g_{0 i}^{-1} g_{0 j}\right)$, and the elements $g_{0 i}$ can then be chosen independently. From (4.1), the group of 1-cocycles is then identified with

$$
Z^{1}:=\bigoplus_{i=1}^{n}\left(\mathbb{k}^{\times} \oplus N^{\prime} \oplus \mathbb{Z}\right)
$$

On the other hand, $\Gamma\left(U_{i, \phi}, \mathcal{O}_{U_{i, \phi}}^{\times}\right)=\mathbb{k}^{\times} \oplus N^{\prime}$. A 0-cochain $g=\left(g_{i}\right)_{0 \leqslant i \leqslant n}, g_{i} \in \mathbb{k}^{\times} \oplus N^{\prime}$ then satisfies $(\partial g)_{0 i}=g_{0}^{-1} \mu_{i}^{*}\left(g_{i}\right)$, where $\partial$ denotes Čech coboundary. Given (2.5), we can then view $\partial$ 
as a map

$$
C^{0}:=\bigoplus_{i=0}^{n}\left(\mathbb{k}^{\times} \oplus N^{\prime}\right) \rightarrow Z^{1}, \quad\left(c_{i}, n_{i}\right)_{0 \leqslant i \leqslant n} \mapsto\left(c_{i} c_{0}^{-1}, n_{i}-n_{0},\left[n_{i}, e_{i}\right]\right)_{1 \leqslant i \leqslant n}
$$

Thus modulo $\partial\left(C^{0}\right)$, every element of $Z^{1}$ is equivalent to some $\left(1,0, a_{i}\right)_{1 \leqslant i \leqslant n}$. Thus $Z^{1} / \partial\left(C^{0}\right)$ is isomorphic to $\mathbb{Z}^{n} /\left(\partial\left(C^{0}\right) \cap \mathbb{Z}^{n}\right)$, where $\mathbb{Z}^{n} \subset Z^{1}$ via the last component for each $i$. But $\partial\left(C^{0}\right) \cap \mathbb{Z}^{n}$ consists of the coboundaries of elements $\left(1, n_{0}\right)_{0 \leqslant i \leqslant n}$, and the coboundary of such an element is $\left(1,0,\left[n_{0}, e_{i}\right]\right)_{1 \leqslant i \leqslant n}$. If we identify $\mathbb{Z}^{n}$ with $M^{\circ}$ using the basis $f_{i}$, then with $n_{0}=e_{j}$, we obtain the element of $M^{\circ}$ given by

$$
\sum_{i=1}^{n}\left[e_{j}, e_{i}\right] f_{i}=\sum_{i=1}^{n}\left\{e_{j}, e_{i}\right\} e_{i}^{*}=p^{*}\left(e_{j}\right) .
$$

This proves the result.

Remark 4.2. We note that the calculations in the proof above demonstrate easily how $X_{e}$ (and hence $X$ and $\mathcal{X}$ ) can fail to be separated. Indeed, suppose that $e_{i}, e_{j}$ agree after projection to $N / K$. In particular, $\left[n, e_{i}\right]=\left[n, e_{j}\right]$ for any $n \in N$ and $\left[e_{j}, e_{i}\right]=0$. Thus $\mu_{j} \circ \mu_{i}^{-1}$ is the identity on $\mathbb{k}[N / K] \cong \mathcal{X}_{\mu_{i}(\mathbf{s}), e} \cong \mathcal{X}_{\mu_{j}(\mathbf{s}), e}$, but $U_{i j, e}$ is a proper subset of $\mathcal{X}_{\mu_{i}(\mathbf{s}), e}$. So the two tori are glued via the identity across a proper open subset of each torus, and we obtain a non-separated scheme.

Construction 4.3. We now recall the construction of the universal torsor over a scheme $X$ with finitely generated Picard group. Ideally, we would like to define the universal torsor as the affine scheme over $X$

$$
U T_{X}:=\operatorname{Spec} \bigoplus_{\mathcal{L} \in \operatorname{Pic} X} \mathcal{L}
$$

However, the quasi-coherent sheaf of $\mathcal{O}_{X}$-modules appearing here does not have a natural algebra structure, since elements of Pic $X$ represent isomorphism classes of line bundles. If Pic $X$ is in fact a free abelian group, we can proceed as in [HK00] and choose a set of line bundles $\mathcal{L}_{1}, \ldots, \mathcal{L}_{n}$ whose isomorphism classes form a basis for the Picard group, and write, for $\nu \in \mathbb{Z}^{n}$, $\mathcal{L}^{\nu}:=\bigotimes_{i=1}^{n} \mathcal{L}_{i}^{\nu_{i}}$. Then $\bigoplus_{\nu \in \mathbb{Z}^{n}} \mathcal{L}^{\nu}$ does have a natural algebra structure.

If Pic $X$ has torsion, then we need to make use of the definition given in [BH03, §3]. We can choose a sufficiently fine open cover $\mathfrak{U}$ of $X$ such that every isomorphism class of line bundle on $X$ is represented by a Cech 1 -cocycle for $\mathcal{O}_{X}^{\times}$with respect to this cover. Denoting the set of Čech 1-cocycles as $Z^{1}\left(\mathfrak{U}, \mathcal{O}_{X}^{\times}\right)$, we choose a finitely generated subgroup $\Lambda \subseteq Z^{1}\left(\mathfrak{U}, \mathcal{O}_{X}^{\times}\right)$. If for $\lambda \in \Lambda$, we denote by $\mathcal{L}_{\lambda}$ the corresponding line bundle, we can choose $\Lambda$ so that the natural map $\Lambda \rightarrow \operatorname{Pic} X, \lambda \mapsto\left[\mathcal{L}_{\lambda}\right]$, is surjective. Then multiplication gives a sheaf of $\mathcal{O}_{X}$-algebras structure to $\mathcal{R}:=\bigoplus_{\lambda \in \Lambda} \mathcal{L}_{\lambda}$

To obtain the universal torsor, we need to define an ideal $\mathcal{I} \subseteq \mathcal{R}$ generated by relations coming from isomorphisms $\mathcal{L}_{\lambda} \cong \mathcal{L}_{\lambda^{\prime}}$. However, these isomorphisms must be chosen carefully, so [BH03] defines the notion of a shifting family. Let $\Lambda_{0}=\operatorname{ker}(\Lambda \rightarrow \operatorname{Pic} X)$. A shifting family is a set of $\mathcal{O}_{X}$-module isomorphisms $\left\{\rho_{\lambda}: \mathcal{R} \rightarrow \mathcal{R} \mid \lambda \in \Lambda_{0}\right\}$ such that

(i) the isomorphism $\rho_{\lambda}$ maps $\mathcal{L}_{\lambda^{\prime}}$ to $\mathcal{L}_{\lambda^{\prime}+\lambda}$, for every $\lambda \in \Lambda_{0}, \lambda^{\prime} \in \Lambda$;

(ii) for every $\lambda_{1}, \lambda_{2} \in \Lambda_{0}, \rho_{\lambda_{1}+\lambda_{2}}=\rho_{\lambda_{1}} \circ \rho_{\lambda_{2}}$;

(iii) if $f, g$ are sections of $\mathcal{L}_{\lambda_{1}}, \mathcal{L}_{\lambda_{2}}$, respectively, for $\lambda_{1}, \lambda_{2} \in \Lambda$, and $\lambda \in \Lambda_{0}$, we have $\rho_{\lambda}(f g)=$ $f \rho_{\lambda}(g)$. 


\section{BiRATIONAL GEOMETRY OF CLUSTER ALGEBRAS}

A shifting family defines a sheaf of ideals $\mathcal{I} \subseteq \mathcal{R}$ such that $\mathcal{I}(U)$ is generated by elements of the form $f-\rho_{\lambda}(f)$ for $f \in \mathcal{R}(U), \lambda \in \Lambda_{0}$.

Given a shifting family, the universal torsor is then defined to be $U T_{X}:=\operatorname{Spec} \mathcal{R} / \mathcal{I}$. A priori $U T_{X}$ depends on the choice of shifting family (although [BH03] proves that any two choices are isomorphic provided that $\mathbb{k}$ is algebraically closed and $\Gamma\left(X, \mathcal{O}_{X}^{\times}\right)=\mathbb{k}^{\times}$; see Lemma 3.7 of [BH03]). If Pic $X$ is torsion free, then this ambiguity disappears. Thus, in general, we will talk about a choice of universal torsor.

Given a seed $\mathbf{s}$ and $t \in T_{M}$, let $A_{t}$ (respectively, $A_{\text {prin }}$ ) be the variety defined by gluing together the seed tori $\mathcal{A}_{\text {prin }, \mathbf{s}, t}, \mathcal{A}_{\text {prin }, \mu_{i}(\mathbf{s}), t}$ (respectively, $\left.\mathcal{A}_{\text {prin,s }}, \mathcal{A}_{\text {prin }, \mu_{i}(\mathbf{s})}\right), 1 \leqslant i \leqslant n$, analogously to $X$.

Theorem 4.4. (i) Let $t \in T_{M}, \phi=i(t) \in T_{K^{*}}$ (see (2.14)). The torsor $p_{t}: A_{t} \rightarrow X_{\phi}$ of construction 2.13 is a universal torsor for $X_{\phi}$. For very general $t, p_{t}: \mathcal{A}_{t}^{\mathrm{ft}} \rightarrow \mathcal{X}_{\phi}^{\mathrm{ft}}$ is a universal torsor for $\mathcal{X}_{\phi}^{\mathrm{ft}}$.

(ii) For $m \in M^{\circ}$, let $\mathcal{L}_{m}$ denote the line bundle on $X$ associated with $m$ under the identification $\operatorname{Pic}(X) \cong M^{\circ} / p^{*}(N)$. Specifically, $\mathcal{L}_{m}$ is the representative of the isomorphism class given by the Cech 1-cocycle represented by $m \in M^{\circ}$ in the proof of Theorem 4.1. Then

$$
A_{\text {prin }}=\operatorname{Spec} \bigoplus_{m \in M^{\circ}} \mathcal{L}_{m}
$$

Furthermore, the line bundle $\mathcal{L}_{m}$ on $X$ extends to a line bundle $\mathcal{L}_{m}$ on $\mathcal{X}^{\mathrm{ft}}$, and similarly

$$
\mathcal{A}_{\text {prin }}^{\mathrm{ft}}=\operatorname{Spec} \bigoplus_{m \in M^{\circ}} \mathcal{L}_{m}
$$

(using the same finite subtrees of $\mathfrak{T}$ to define both $\mathcal{A}_{\mathrm{prin}}^{\mathrm{ft}}$ and $\mathcal{X}^{\mathrm{ft}}$ ).

Proof. We first prove the statements for $A_{\text {prin }}, X$ and $A_{t}, X_{\phi}$. Continuing with the notation of the proof of Theorem 4.1 and construction 4.3, we take the open covers

$$
\begin{aligned}
\mathfrak{U} & =\left\{\mathcal{X}_{\mathbf{s}}\right\} \cup\left\{\mathcal{X}_{\mu_{i}(\mathbf{s})} \mid 1 \leqslant i \leqslant n\right\}=\left\{U_{i} \mid 0 \leqslant i \leqslant n\right\}, \\
\mathfrak{U}_{\phi} & =\left\{\mathcal{X}_{\mathbf{s}, \phi}\right\} \cup\left\{\mathcal{X}_{\mu_{i}(\mathbf{s}), \phi} \mid 1 \leqslant i \leqslant n\right\}=\left\{U_{i, \phi} \mid 0 \leqslant i \leqslant n\right\},
\end{aligned}
$$

as usual. We saw in the proof of Theorem 4.1 that $M^{\circ}$ is naturally identified with a subgroup of both $Z^{1}\left(\mathfrak{U}, \mathcal{O}_{X}^{\times}\right)$and $Z^{1}\left(\mathfrak{U}_{\phi}, \mathcal{O}_{X_{\phi}}^{\times}\right)$. Taking the subgroup $\Lambda$ of this cocycle group to be $M^{\circ}$, we obtain

$$
\Lambda_{0}=\operatorname{ker}\left(\Lambda \rightarrow \operatorname{Pic}\left(X_{\phi}\right)\right)=\operatorname{ker}\left(M^{\circ} \rightarrow M^{\circ} / p^{*}(N)\right)=p^{*}(N) .
$$

This then gives rise to a sheaf of $\mathcal{O}_{X}$-algebras $\mathcal{R}=\bigoplus_{\lambda \in \Lambda} \mathcal{L}_{\lambda}$ and a sheaf of $\mathcal{O}_{X_{\phi}}$-algebras $\mathcal{R}_{\phi}$ defined by the same formula. For the two cases, we have the maps $\tilde{p}: A_{\text {prin }} \rightarrow X$ and $p_{t}: A_{t} \rightarrow X_{\phi}$ of construction 2.13. Noting that

$$
\begin{gathered}
U_{i, A_{\text {prin }}}:=\tilde{p}^{-1}\left(U_{i}\right)= \begin{cases}\mathcal{A}_{\text {prin }, \mathbf{s}} & \text { for } i=0, \\
\mathcal{A}_{\text {prin }, \mu_{i}(\mathbf{s})} & \text { for } i>0,\end{cases} \\
U_{i, A_{t}}:=p_{t}^{-1}\left(U_{i, \phi}\right)= \begin{cases}\mathcal{A}_{\text {prin }, \mathbf{s}, t} & \text { for } i=0, \\
\mathcal{A}_{\text {prin }, \mu_{i}(\mathbf{s}), t} & \text { for } i>0,\end{cases}
\end{gathered}
$$

we see that the morphisms $\tilde{p}, p_{t}$ are affine. Thus to prove both parts of the theorem, it is sufficient to construct morphisms of sheaves of $\mathcal{O}_{X}$-algebras or $\mathcal{O}_{X_{\phi}}$-algebras

$$
\psi: \mathcal{R} \rightarrow \tilde{p}_{*} \mathcal{O}_{A_{\text {prin }}}, \quad \psi_{\phi}: \mathcal{R}_{\phi} \rightarrow p_{*} \mathcal{O}_{A_{t}}
$$




\section{Mark Gross, Paul Hacking and Sean Keel}

such that $\psi$ is an isomorphism and the kernel of $\psi_{\phi}$ is an ideal $\mathcal{I}$ arising from a shifting family.

First, by construction, $\left.\mathcal{R}_{\phi}\right|_{U_{i, \phi}} \cong \bigoplus_{m \in M^{\circ}} \mathcal{O}_{U_{i, \phi}} e_{m}$, and the transition function on $U_{0 i, \phi}$ for the generator $e_{m}$ is $\left(1+z^{e_{i}}\right)^{-\left\langle d_{i} e_{i}, m\right\rangle}$. The same formulae hold for $\mathcal{R}$. Let $I_{\phi} \subseteq \Gamma\left(U_{i}, \mathcal{O}_{U_{i}}\right)=\mathbb{k}[N]$ be the ideal of the fibre $U_{i, \phi} \subseteq U_{i}$, and let $I_{t} \subseteq \Gamma\left(U_{i, A_{\text {prin }}}, \mathcal{O}_{U_{i, A_{\text {prin }}}}\right)=\mathbb{k}\left[\widetilde{M}^{\circ}\right]$ be the ideal of the fibre $U_{i, A_{t}}$ of $\pi: U_{i, A_{\text {prin }}} \rightarrow T_{M}$ over $t$. Then $\left.\mathcal{R}_{\phi}\right|_{U_{i, \phi}}$ is the quasi-coherent sheaf associated with the free $\mathbb{k}[N] / I_{\phi}$-module with basis $\left\{e_{m} \mid m \in M^{\circ}\right\}$, while $\left.\mathcal{R}\right|_{U_{i}}$ is the quasi-coherent sheaf associated with the free $\mathbb{k}[N]$-module with the same basis.

Second, note that $\tilde{p}_{*} \mathcal{O}_{U_{i, A} \text { prin }}$ (respectively, $p_{*} \mathcal{O}_{U_{i, A_{t}}}$ ) is the quasi-coherent sheaf associated with the $\mathbb{k}[N]$-algebra $\mathbb{k}\left[\widetilde{M}^{\circ}\right]$ (respectively, the $\mathbb{k}[N] / I_{\phi^{-}}$-algebra $\left.\mathbb{k}\left[\widetilde{M}^{\circ}\right] / I_{t}\right)$. The algebra structure is given by the map $N \rightarrow \widetilde{M}^{\circ}, n \mapsto\left(p^{*}(n), n\right)$. There are natural maps $\left.\mathcal{R}\right|_{U_{i}} \rightarrow \tilde{p}_{*} \mathcal{O}_{U_{i, A} \text { prin }}$ and $\left.\mathcal{R}_{\phi}\right|_{U_{i, \phi}} \rightarrow\left(p_{t}\right)_{*} \mathcal{O}_{U_{i, A_{t}}}$ induced by the maps of $\mathbb{k}[N]$ - or $\mathbb{k}[N] / I_{\phi}$-modules given by $e_{m} \mapsto z^{(m, 0)}$. We first check that these maps respect the transition maps. We do this for the case of $A_{t} \rightarrow X_{\phi}$, the case of $A_{\text {prin }} \rightarrow X$ being identical. On $U_{0 i, \phi}, e_{m}$ is glued to $\left(1+z^{e_{i}}\right)^{-\left\langle d_{i} e_{i}, m\right\rangle} e_{m}$ as observed above, while $z^{(m, 0)} \in \mathbb{k}\left[\widetilde{M}^{\circ}\right] / I_{t}$ is transformed via the $\mathcal{A}$ mutation $\mu_{i}$. But using (2.6),

$$
\frac{\mu_{i}^{*}\left(z^{(m, 0)}\right)}{z^{(m, 0)}}=\left(1+z^{\left(v_{i}, e_{i}\right)}\right)^{-\left\langle d_{i} e_{i}, m\right\rangle} .
$$

This can be viewed via $p_{t}^{*}$ as the function on $U_{0 i, \phi}$ given by $\left(1+z^{e_{i}}\right)^{-\left\langle d_{i} e_{i}, m\right\rangle}$. This shows that the transition maps match up, and we obtain the desired map (4.2).

Note that $\psi$ is easily seen to be an isomorphism. On the other hand, the kernel $\mathcal{I}$ of $\psi_{\phi}$ is generated on $U_{i, \phi}$ by elements of $\bigoplus e_{m} \mathbb{k}[N] / I_{\phi}$ of the form $e_{m}-e_{m+p^{*}(n)} z^{-n}$ for $m \in M^{\circ}, n \in N$. This arises from the family of identifications $\left\{\rho_{p^{*}(n)}\right\}$ defined by $\rho_{p^{*}(n)}\left(e_{m}\right)=e_{m+p^{*}(n)} z^{-n}$. This is easily checked to be a shifting family.

This completes the proof for $A_{\text {prin }}, X$ and $A_{t}, X_{\phi}$. To prove the result for $\mathcal{A}_{\text {prin }}^{\mathrm{ft}}, \mathcal{X}^{\mathrm{ft}}$, etc., one just notes that the corresponding spaces are equal to $A_{\text {prin }}, X$, etc. outside codimension two sets.

Definition 4.5. Given a choice of shifting family for a scheme $X$ over a field $\mathbb{k}$ with finitely generated Picard group, the Cox ring $\operatorname{Cox}(X)$ of $X$ is the $\mathbb{k}$-algebra of global sections of $\mathcal{R} / \mathcal{I}$. If Pic $X$ is free, this coincides with the usual definition

$$
\operatorname{Cox}(X)=\bigoplus_{\nu} \Gamma\left(X, \mathcal{L}^{\nu}\right)
$$

after a choice of line bundles $\mathcal{L}_{1}, \ldots, \mathcal{L}_{n}$ whose isomorphism classes give a basis for Pic $X$.

COROLlary 4.6. (i) The upper cluster algebra with principal coefficients is isomorphic to

$$
\bigoplus_{m \in M^{\circ}} \Gamma\left(X, \mathcal{L}_{m}\right)
$$

(ii) If the initial seed is totally coprime, the upper cluster algebra is isomorphic to the Cox ring of $X_{e}$.

(iii) For $t \in T_{M}$ very general, $\Gamma\left(\mathcal{A}_{t}, \mathcal{O}_{\mathcal{A}_{t}}\right)$ is isomorphic to the Cox ring of $X_{i(t)}$.

Proof. This follows because under the hypotheses, the upper cluster algebra $\Gamma\left(\mathcal{A}, \mathcal{O}_{\mathcal{A}}\right)$ coincides with $\Gamma\left(A, \mathcal{O}_{A}\right)$ by Theorem 3.9 and Lemma 3.5. The latter algebra has the desired description by Theorem 4.4. The principal coefficient case is similar. 


\section{BiRATIONAL GEOMETRY OF CLUSTER ALGEBRAS}

Corollary 4.7. If $\operatorname{Pic}(X)$ is torsion free (that is, if $M^{\circ} / p^{*}(N)$ is torsion free), then the upper cluster algebra with principal coefficients $\Gamma\left(\mathcal{A}_{\text {prin }}, \mathcal{O}_{\mathcal{A}_{\text {prin }}}\right)$ and for very general $t$, the upper cluster algebra with general coefficients $\Gamma\left(\mathcal{A}_{t}, \mathcal{O}_{\mathcal{A}_{t}}\right)$ are factorial. If the initial seed is totally coprime, then the upper cluster algebra $\Gamma\left(\mathcal{A}, \mathcal{O}_{\mathcal{A}}\right)$ is factorial.

Proof. For the cases other than $\mathcal{A}_{\text {prin }}$, this follows from Theorem 1.1 of [Arz09], (see also [BH03, Proposition 8.4].)

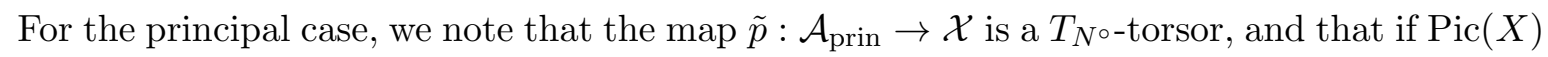
is torsion-free, then $\operatorname{Pic}(X)^{*} \subseteq N^{\circ}$ and $T_{\operatorname{Pic}(X)^{*}}$ is a subtorus of $T_{N^{\circ}}$. Write $X^{\prime}=A_{\operatorname{prin}} / T_{\operatorname{Pic}(X)^{*}}$. Note that $X^{\prime}=$ Spec $\bigoplus_{m \in p^{*}(N)} \mathcal{L}_{m}$. But $\mathcal{L}_{m} \cong \mathcal{O}_{X}$ as a line bundle for $m \in p^{*}(N)$, so $X^{\prime} \rightarrow X$

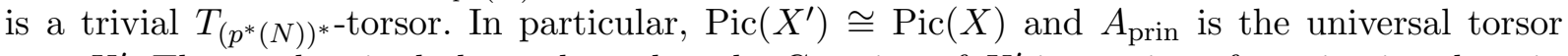
over $X^{\prime}$. The results cited above show that the Cox ring of $X^{\prime}$ is a unique factorization domain (UFD), so the upper cluster algebra with principal coefficients is also a UFD.

\section{The $\mathcal{X}$ variety in the $\operatorname{rank} \epsilon=2$ case}

In this section we will fix seed data as usual, with the same assumptions as in the previous section, namely that there are no frozen variables and that no row (or column) of $\epsilon$ is zero. We will assume furthermore $\operatorname{rank} \epsilon=2$, that is, $\operatorname{rank} K=\operatorname{rank} N-2$. In this case, the morphism $\mathcal{X} \rightarrow T_{K^{*}}$ is a flat family of two-dimensional schemes (flatness following from the fact that the maps $\mathcal{X}_{\mathbf{s}} \rightarrow T_{K^{*}}$ are flat for each seed). We can use the description of the $\mathcal{X}$ variety given in $\S 3.2$ to develop a geometric feeling for this family.

Now $K^{\perp} \subseteq M$ is a saturated rank two sublattice by the assumption on the rank of $\epsilon$. Furthermore, $d_{i} v_{i} \in K^{\perp}$ for each $i$ and these vectors are non-zero by the assumption on $\epsilon$. Choose a complete non-singular fan $\bar{\Sigma}$ in $K^{\perp}$ such that each $-d_{i} v_{i}$ generates a ray of $\bar{\Sigma}$. Via the inclusion $K^{\perp} \subseteq M, \bar{\Sigma}$ also determines a fan in $M$, which we denote by $\Sigma$. Note that $\Sigma$ contains the fan of one-dimensional cones $\Sigma_{\mathbf{s}, \mathcal{X}}$. Then the projection $M \rightarrow K^{*} \cong M / K^{\perp}$ induces a map

$$
\bar{\lambda}: \operatorname{TV}(\Sigma) \rightarrow T_{K^{*}}
$$

each of whose fibres is a complete toric surface $\mathrm{TV}(\bar{\Sigma})$; we in fact have non-canonically $\mathrm{TV}(\Sigma) \cong$ $\mathrm{TV}(\bar{\Sigma}) \times T_{K^{*}}$, arising from a choice of splitting $M=K^{\perp} \oplus K^{*}$.

Let $D_{i}$ denote the divisor of $\mathrm{TV}(\Sigma)$ corresponding to the ray generated by $-d_{i} v_{i}$. For each $i$ we obtain a (possibly non-reduced) hypersurface $Z_{i} \subseteq D_{i}$ given by

$$
Z_{i}:=D_{i} \cap \bar{V}\left(\left(1+z^{e_{i}}\right)^{\text {ind } d_{i} v_{i}}\right)
$$

as in (3.2).

Lemma 5.1. The underlying closed subset of $Z_{i}$ is the image of a section $q_{i}: T_{K^{*}} \rightarrow \mathrm{TV}(\Sigma)$ of $\bar{\lambda}$ if and only if the image of $e_{i}$ in $N / K$ is primitive.

Proof. A choice of splitting $M=K^{\perp} \oplus K^{*}$ gives a dual splitting $N \cong N / K \oplus K$. Write $e_{i}=\left(e_{i}^{\prime}, e_{i}^{\prime \prime}\right)$ under this splitting. The monomial $z^{e_{i}}$ is non-vanishing on $D_{i}$ as $\left\langle e_{i},-d_{i} v_{i}\right\rangle=0$. Then restricting $z^{e_{i}}$ to $\bar{\lambda}^{-1}(\phi) \cap D_{i}$ for some $\phi \in T_{K^{*}}$, we obtain a monomial $\left(z^{e_{i}^{\prime \prime}}(\phi)\right) \cdot z^{e_{i}^{\prime}} \in \mathbb{k}\left[\left(d_{i} v_{i}\right)^{\perp}\right]$, where $\left(d_{i} v_{i}\right)^{\perp}$ is a sublattice of $N / K$. Thus $Z_{i} \cap \bar{\lambda}^{-1}(\phi) \cap D_{i}$ consists of a single point if and only if $e_{i}^{\prime}$ is primitive; that is, the image of $e_{i}$ is primitive in $N / K$.

The following is an enhanced restatement of Theorem 3.9. 


\section{Mark Gross, Paul Hacking and Sean Keel}

Lemma 5.2. Let $\mathcal{Y} \rightarrow T_{K^{*}}$ be the flat family of surfaces obtained by blowing up the subschemes $Z_{i} \subseteq \mathrm{TV}(\Sigma)$ in some order. Let $\mathcal{D} \subseteq \mathcal{Y}$ be the proper transform of the toric boundary of $\mathrm{TV}(\Sigma)$, $\lambda: \mathcal{Y} \backslash \mathcal{D} \rightarrow T_{K^{*}}$ the induced map.

(i) The variety $\mathcal{X}^{\mathrm{ft}}$ is isomorphic to $\mathcal{Y} \backslash \mathcal{D}$ outside a set of codimension at least two.

(ii) If $\phi \in T_{K^{*}}$ is very general (that is, in the complement of a countable union of proper closed subsets), then $\lambda^{-1}(\phi)$ is isomorphic to the fibre $\mathcal{X}_{\phi}^{\mathrm{ft}}$ of $\mathcal{X}^{\mathrm{ft}} \rightarrow T_{K^{*}}$ outside a set of codimension two.

Proof. Statement (i) is immediate from Theorem 3.9, observing that blowing up the $Z_{i}$ in some order differs only in codimension two with the blowup of the subscheme $\bigcup_{i} Z_{i}$. For statement (ii), we first use the explicit description of $X$ given at the beginning of $\S 4$. Indeed, $X$ is obtained by gluing together tori $U_{i}, 0 \leqslant i \leqslant n$ as described explicitly in the proof of Theorem 4.1. Denote by $U_{i, \phi}, U_{i j, \phi}$ the fibres of $U_{i}, U_{i j} \rightarrow T_{K^{*}}$ over $\phi$. If $Z_{i} \cap Z_{j} \cap \bar{\lambda}^{-1}(\phi)=\emptyset$, it is then easy to see that the maximal open set of the domain for which the map $\left.\varphi_{i j}\right|_{U_{i, \phi}}: U_{i, \phi}-\rightarrow U_{j, \phi}$ is an isomorphism is precisely $U_{i j, \phi}$. Thus $X_{\phi}$ is constructed as the space $X$ is in Lemma 3.5. The schemes $Z_{i}$ in that construction coincide with the schemes $\bar{\lambda}^{-1}(\phi) \cap Z_{i}$. Thus, provided $\phi$ does not lie in $\bar{\lambda}\left(Z_{i} \cap Z_{j}\right)$ for any $i, j$, Lemma 3.5 applies to show that there is an open immersion $X_{\phi} \rightarrow \lambda^{-1}(\phi)$ which is an isomorphism outside a codimension two subset of $\lambda^{-1}(\phi)$.

To complete the argument, we follow the proofs of Lemma 3.8 and Theorem 3.9. If $\mathbf{s}^{\prime}=\mu_{k}(\mathbf{s})$ and $X^{\prime}, \mathcal{Y}^{\prime}, Z_{i}^{\prime}$, etc., are constructed using the seed $\mathbf{s}^{\prime}$, then the argument of Lemma 3.8 shows that provided $\phi \notin \lambda\left(Z_{i} \cap Z_{j}\right), \lambda^{\prime}\left(Z_{i}^{\prime} \cap Z_{j}^{\prime}\right)$ for any pair $i \neq j, \lambda^{-1}(\phi)$ is isomorphic to $\left(\lambda^{\prime}\right)^{-1}(\phi)$ off codimension two. Thus $X_{\phi}$ and $X_{\phi}^{\prime}$ are isomorphic outside a set of codimension two, and the argument is finished as in Theorem 3.9.

Thus the family $\mathcal{X}^{\mathrm{ft}} \rightarrow T_{K^{*}}$ can be thought of, away from codimension two, as a family of surfaces obtained by blowing up a collection of points on the boundary of a toric variety, and then deleting the proper transform of the boundary. In general, since these points are being blown up with multiplicity, $\mathcal{Y} \backslash \mathcal{D}$ can be singular. We will first see that any surface obtained via blowups on the boundary of a toric surface is deformation equivalent to any surface in the family $\mathcal{Y} \rightarrow T_{K^{*}}$ constructed using some seed.

Construction 5.3. Let $\bar{Y}$ be a complete non-singular toric surface, with toric boundary $\bar{D}$, given by a fan $\bar{\Sigma}$ in a lattice $\bar{N} \cong \mathbb{Z}^{2}$. Choose a collection of irreducible boundary divisors $\bar{D}_{1}, \ldots, \bar{D}_{n}$ (possibly with repetitions) and let $w_{i} \in \bar{N}$ be the primitive generator of the ray corresponding to $\bar{D}_{i}$. Fix positive integers $\nu_{i}, 1 \leqslant i \leqslant n$. Suppose that $w_{1}, \ldots, w_{n}$ generate $\bar{N}$.

We will use this data to construct seed data, as follows. Set $N=\mathbb{Z}^{n}$ with basis $\left\{e_{i}\right\}$ and let $M$ be the dual lattice as usual. Define a map $\psi: N \rightarrow \bar{N}$ by $e_{i} \mapsto w_{i}$. By assumption, $\psi$ is surjective. Choose an isomorphism $\bigwedge^{2} \bar{N} \cong \mathbb{Z}$. The map $\varphi: \bar{N} \rightarrow M$ given by $\bar{n} \mapsto(n \mapsto \psi(n) \wedge \bar{n})$ gives a primitive embedding of the lattice $\bar{N}$ into $M$ by the surjectivity of $\psi$. Let $\nu=\operatorname{gcd}\left(\nu_{1}, \ldots, \nu_{n}\right)$. We then obtain an integer-valued skew-symmetric form $\{\cdot, \cdot\}$ on $N$ by setting

$$
\left\{n_{1}, n_{2}\right\}=\nu \psi\left(n_{1}\right) \wedge \psi\left(n_{2}\right) \in \mathbb{Z} .
$$

Note that $\operatorname{ker} \psi$ coincides with $K=\{n \in N \mid\{n, \cdot\}=0\}$. Set $d_{i}=\nu_{i} / \nu$. This gives us seed data $\left\{e_{i}\right\}$ for the fixed data $N=N_{\mathrm{uf}},\{\cdot, \cdot\}$ and $\left\{d_{i}\right\}$.

We now analyze the family $\mathcal{Y} \rightarrow T_{K^{*}}$ arising from this seed data. Using the inclusion $\varphi$, we write $\Sigma$ for the fan $\bar{\Sigma}$ as a fan in $M$. We write $D_{i}$ for the toric divisor of $\operatorname{TV}(\Sigma)$ corresponding 


\section{BiRATIONAL GEOMETRY OF CLUSTER ALGEBRAS}

to the ray generated by $w_{i}$. We note that with

$$
v_{i}=p^{*}\left(e_{i}\right)=\left\{e_{i}, \cdot\right\}=-\nu \varphi\left(\psi\left(e_{i}\right)\right),
$$

we have $-d_{i} v_{i}=\nu_{i} \varphi\left(w_{i}\right)$. As $\psi$ is surjective, $N / K \cong \bar{N}$, and the image $w_{i}$ of each $e_{i}$ in $N / K$ is primitive. Thus by Lemma 5.1, the closed sets $Z_{i}$ are images of sections of $D_{i} \rightarrow T_{K^{*}}$. It then follows by Lemma 5.2 that the general fibre of $\lambda: \mathcal{Y} \rightarrow T_{K^{*}}$ is obtained by blowing up $\bar{Y}$ at a collection of points $p_{1}, \ldots, p_{n}$, with $p_{i} \in \bar{D}_{i}$ taken with multiplicity $\nu_{i}$.

We now consider the special case that all $\nu_{i}=1$. First we note the following.

\section{Proposition 5.4. Giving}

- fixed data with rank $N=n$, no frozen variables, $d_{i}=1$ for all $i$ and such that $\{\cdot, \cdot\}$ has rank two and the induced non-degenerate skew-symmetric pairing on $N / K$ is unimodular, and

- a seed $\mathbf{s}$ for this fixed data such that the image of each $e_{i}$ in $N / K$ is primitive

is equivalent to giving primitive vectors $w_{1}, \ldots, w_{n} \in \bar{N}$ where $\bar{N}$ is a rank two lattice and for which $w_{1}, \ldots, w_{n}$ generate $\bar{N}$.

Proof. Construction 5.3 explains how to pass from the data of the vectors $w_{i}$ to the fixed and seed data. Here we take $\nu_{i}=1$ for all $i$ in that construction. Conversely, given fixed and seed data as in the proposition, we take $\bar{N}=N / K, w_{i}$ the image of each $e_{i}$. The only unimodular integral skew-symmetric pairing on $\bar{N}$, up to sign, is given by $\left\{n_{1}, n_{2}\right\}=n_{1} \wedge n_{2}$, after a choice of identification $\bigwedge^{2} \bar{N} \cong \mathbb{Z}$. Thus after making a suitable choice of identification, the given pairing $\{\cdot, \cdot\}$ on $N$ agrees with the one described in construction 5.3.

Continuing with the notation above, with $\nu_{1}=\cdots=\nu_{n}=1$, consider the family $\lambda: \mathcal{Y} \rightarrow T_{K^{*}}$ of blown up toric surfaces. In this case, a general fibre $\lambda^{-1}(\phi)$ for $\phi \in T_{K^{*}}$ is obtained by blowing up reduced points on the non-singular part of the toric boundary of $\bar{Y}$. A fibre $(Y, D)=\left(\mathcal{Y}_{\phi}, \mathcal{D}_{\phi}\right)$ is what we call a Looijenga pair in [GHK15]. Given a cyclic ordering of the irreducible components of $D=D_{1}^{\prime}+\cdots+D_{r}^{\prime}$, one gets a canonical identification of the identity component $\operatorname{Pic}^{0}(D)$ of Pic $D$ with $\mathbb{G}_{m}$; see [GHK15, Lemma 2.1]. (We note that the divisors $D_{1}, \ldots, D_{n}$ are a possibly proper subset of $D_{1}^{\prime}, \ldots, D_{r}^{\prime}$, and the former occur with repetitions and need not be cyclically ordered). Furthermore, we define $D^{\perp} \subseteq \operatorname{Pic}(Y)$ by

$$
D^{\perp}:=\left\{H \in \operatorname{Pic}(Y) \mid H \cdot D_{i}^{\prime}=0 \quad \forall i\right\} .
$$

Then the period point of $(Y, D)$ is the element of $\operatorname{Hom}\left(D^{\perp}, \operatorname{Pic}^{0}(D)\right)$ given by restriction.

TheOrem 5.5. Let $\pi: Y \rightarrow \bar{Y}$ be the blowup describing $Y$, with exceptional divisors $E_{1}, \ldots, E_{n}$ over $Z_{1} \cap \bar{\lambda}^{-1}(\phi), \ldots, Z_{n} \cap \bar{\lambda}^{-1}(\phi)$. Then there is a natural isomorphism

$$
K \rightarrow D^{\perp}, \quad \sum a_{i} e_{i} \mapsto \pi^{*} C-\sum a_{i} E_{i},
$$

where $C$ is the unique divisor class such that

$$
C \cdot D_{j}^{\prime}=\sum_{i: D_{i}=D_{j}^{\prime}} a_{i} .
$$

Under this identification and the canonical identification $\operatorname{Pic}^{0}(D) \cong \mathbb{G}_{m}$, the period point of $(Y, D)$ in $\operatorname{Hom}\left(D^{\perp}, \operatorname{Pic}^{0}(D)\right)$ coincides with $\phi \in T_{K^{*}}=\operatorname{Hom}\left(K, \mathbb{G}_{m}\right)$. 


\section{Mark Gross, Paul Hacking and Sean Keel}

Proof. Recall the standard description of the second homology group of the toric variety $\bar{Y}$ :

$$
0 \rightarrow H_{2}(\bar{Y}, \mathbb{Z}) \rightarrow \mathbb{Z}^{r} \rightarrow \bar{N} \rightarrow 0,
$$

where the map $\mathbb{Z}^{r} \rightarrow \bar{N}$ takes the $i$ th generator of $\mathbb{Z}^{r}$ to the primitive generator of the ray of $\bar{\Sigma}$ corresponding to the divisor $\bar{D}_{i}^{\prime}$. The inclusion $H_{2}(\bar{Y}, \mathbb{Z}) \hookrightarrow \mathbb{Z}^{r}$ is given by $\alpha \mapsto\left(\alpha \cdot D_{i}^{\prime}\right)_{1 \leqslant i \leqslant r}$. Since $\bar{Y}$ is a non-singular proper rational surface, we have $H_{2}(\bar{Y}, \mathbb{Z}) \cong \operatorname{Pic}(\bar{Y})$. In particular, if $\sum a_{i} e_{i}$ is an element of $K$, then $\left(\sum_{j: D_{j}=D_{i}^{\prime}} a_{j}\right)_{1 \leqslant i \leqslant r} \in H_{2}(\bar{Y}, \mathbb{Z})$. Thus there is a unique element $C \in H_{2}(\bar{Y}, \mathbb{Z}) \cong \operatorname{Pic}(\bar{Y})$ satisfying (5.1). It is then clear that $\pi^{*} C-\sum a_{i} E_{i} \in D^{\perp}$. That this is an isomorphism is easily checked.

We now need to calculate $\left.\mathcal{O}_{Y}\left(\pi^{*} C-\sum a_{i} E_{i}\right)\right|_{D}$. As the identification $\operatorname{Pic}^{0}(D)$ with $\mathbb{G}_{m}$ requires a choice of cyclic ordering of the divisors $D_{i}^{\prime}$, or equivalently of the divisors $\bar{D}_{i}^{\prime}$, we order $w_{1}^{\prime}, \ldots, w_{r}^{\prime}$ clockwise as defined using the choice of isomorphism $\bigwedge^{2} \bar{N} \rightarrow \mathbb{Z}$. In particular, this choice of isomorphism also allows an identification of $\bar{N}$ with $\bar{M}=\operatorname{Hom}(\bar{N}, \mathbb{Z})$, via $n \in \bar{N}$ acting by $n^{\prime} \mapsto\left(n^{\prime} \wedge n\right)$. Thus, in particular, $z^{w_{i}^{\prime}}$ can be viewed as a coordinate on $D_{i}^{\prime}$ which is zero on $p_{i, i+1}$, the intersection point of $D_{i}^{\prime}$ and $D_{i+1}^{\prime}$, and infinite at $p_{i-1, i}$ (all indices taken modulo $r$ ).

We next note that $\left.\mathcal{O}_{\bar{Y}}(C)\right|_{\bar{D}}$ was calculated in the proof of [GHK15, Lemma 2.6(1)]. Let $m_{i} \in D_{i}^{\prime}$ be the point where $z^{w_{i}^{\prime}}$ takes on the value -1 . Then

$$
\left.\mathcal{O}_{\bar{Y}}(C)\right|_{\bar{D}}=\mathcal{O}_{\bar{D}}\left(\sum_{j=1}^{r}\left(C \cdot \bar{D}_{j}^{\prime}\right) m_{j}\right)
$$

Thus we have the same identity for the restriction of $\mathcal{O}_{Y}\left(\pi^{*} C\right)$ to $D$. So if $E_{i} \cap D=p_{i}$, we then have

$$
\mathcal{L}:=\left.\mathcal{O}_{Y}\left(\pi^{*} C-\sum a_{i} E_{i}\right)\right|_{D} \cong \mathcal{O}_{D}\left(-\sum_{i=1}^{n} a_{i} p_{i}+\sum_{j=1}^{r}\left(C \cdot \bar{D}_{j}^{\prime}\right) m_{j}\right) .
$$

This line bundle is described under the isomorphism $\operatorname{Pic}^{0}(D) \cong \mathbb{G}_{m}$ of [GHK15, Lemma 2.6], as follows. We have $\left.\mathcal{L}\right|_{D_{j}^{\prime}}=\mathcal{O}_{D_{j}^{\prime}}\left(\left(C \cdot \bar{D}_{j}^{\prime}\right) m_{j}-\sum_{i: D_{i}=D_{j}^{\prime}} a_{i} p_{i}\right)$. Viewing this trivial line bundle as a subsheaf of the sheaf of rational functions, and using a splitting $M=K^{\perp} \oplus K^{*}, N=N / K \oplus K$ as in the proof of Lemma 5.1, a trivializing section is given by the rational function

$$
\sigma_{j}:=\frac{\prod_{i: D_{i}=D_{j}^{\prime}}\left(z^{w_{j}^{\prime}} \cdot z^{e_{i}^{\prime \prime}}(\phi)+1\right)^{a_{i}}}{\left(z^{w_{j}^{\prime}}+1\right)^{C \cdot \bar{D}_{j}^{\prime}}}
$$

since $Z_{i}$ is given by the equation $z^{e_{i}}+1=0$ and under the choice of splitting $e_{i}=\left(w_{i}, e_{i}^{\prime \prime}\right)$, with $w_{i}=w_{j}^{\prime}$ if $D_{i}=D_{j}^{\prime}$. The image of the line bundle in $\mathbb{G}_{m}$ is

$$
\prod_{j=1}^{r} \sigma_{j+1}\left(p_{j, j+1}\right) / \sigma_{j}\left(p_{j, j+1}\right)=\prod_{i=1}^{n}\left(z^{e_{i}^{\prime \prime}}(\phi)\right)^{a_{i}} .
$$

Remembering that we are viewing $\phi \in \operatorname{Hom}\left(K, \mathbb{G}_{m}\right)$, we see that $z^{e_{i}^{\prime \prime}}(\phi)=\phi\left(e_{i}^{\prime \prime}\right)$. Note that if $\sum a_{i} e_{i} \in K$, we have $\sum a_{i} e_{i}=\sum a_{i} e_{i}^{\prime \prime}$. Thus we see that the element of $\mathbb{G}_{m}$ corresponding to our line bundle is precisely $\phi\left(\sum a_{i} e_{i}\right)$. Thus $\phi$ is the period point of $(Y, D)$.

This shows that the families $\mathcal{Y} \rightarrow T_{K^{*}}$ agree with the universal families of Looijenga pairs constructed in [GHK15].

We can also use the observations above to define an unexpected mutation invariant in the situation of Proposition 5.4. 


\section{Birational GEOMETRY OF CLUSTER ALGEBRAS}

Theorem 5.6. Given fixed data and seed data satisfying the conditions of Proposition 5.4, the isomorphism $K \cong D^{\perp}$ of Theorem 5.5 induces a symmetric integral pairing on $K$ via the intersection pairing on $D^{\perp} \subseteq \operatorname{Pic}(Y)$. This symmetric pairing on $K$ is independent of mutation.

Proof. It is enough to check the independence under a single mutation $\mu_{k}$. So suppose given seeds $\mathbf{s}, \mathbf{s}^{\prime}=\mu_{k}(\mathbf{s})$. These two seeds give rise to families $\mathcal{Y}, \mathcal{Y}^{\prime} \rightarrow T_{K^{*}}$. Fix $\phi \in T_{K^{*}}$ sufficiently general so that the fibres $\mathcal{Y}_{\phi}$ and $\mathcal{Y}_{\phi}^{\prime}$ are both blowups of toric varieties $\bar{Y}, \bar{Y}^{\prime}$ at distinct points. These toric varieties are related by an elementary transformation as follows. Let $\psi: N \rightarrow \bar{N}=N / K$ be the quotient map. If $\bar{\Sigma}, \bar{\Sigma}^{\prime}$ are the fans in $\bar{N}$ defining $\bar{Y}$ and $\bar{Y}^{\prime}$ respectively, then $\bar{\Sigma}^{\prime}$ is obtained from $\bar{\Sigma}$ as follows. First, we can assume that both $\bar{\Sigma}$ and $\bar{\Sigma}^{\prime}$ contain rays generated by $w_{k}$ and $-w_{k}$. Then we can assume that the remaining rays of $\bar{\Sigma}^{\prime}$ are obtained by applying the piecewise linear transformation

$$
T_{k}: \bar{n} \mapsto \bar{n}+\left[\bar{n} \wedge w_{k}\right]_{+} w_{k}
$$

Note, in particular, that this is compatible with (2.3).

The map $\bar{N} \rightarrow \bar{N} / \mathbb{R} w_{k}$ defines $\mathbb{P}^{1}$-fibrations $g: \bar{Y} \rightarrow \mathbb{P}^{1}, g^{\prime}: \bar{Y}^{\prime} \rightarrow \mathbb{P}^{1}$.

By Lemma 3.6, the seed mutation $\mu_{k}: T_{N} \rightarrow T_{N}$ extends to a birational map $\mu_{k}: \mathcal{Y} \rightarrow \mathcal{Y}^{\prime}$ which is an isomorphism outside sets of codimension at least two. In fact, one checks easily from the details of the proof that this birational map restricts to a biregular isomorphism between $Y$ and $Y^{\prime}$. Specifically, this isomorphism is described as follows. Let $p_{1}, \ldots, p_{n}$ be the points of $\bar{Y}$ blown up to obtain $Y$, and $p_{1}^{\prime}, \ldots, p_{n}^{\prime}$ the points of $\bar{Y}^{\prime}$ blown up to obtain $Y^{\prime}$. Then if $Y_{k}, Y_{k}^{\prime}$ denote the blowup of $\bar{Y}, \bar{Y}^{\prime}$ at $p_{k}, p_{k}^{\prime}$, respectively, we already have an isomorphism $\mu_{k}: Y_{k} \rightarrow Y_{k}^{\prime}$, and $p_{i}^{\prime}=\mu_{k}\left(p_{i}\right)$ for $i \neq k$. The isomorphism $\mu_{k}: Y \rightarrow Y^{\prime}$ is then obtained by further blowing up the points $p_{i}, p_{i}^{\prime}$ for $i \neq k$. Furthermore, the composition $Y_{k} \stackrel{\mu_{k}}{\longrightarrow} Y_{k}^{\prime} \rightarrow \bar{Y}^{\prime}$ contracts the proper transform of the curve $F_{k}=g^{-1}\left(g\left(p_{k}\right)\right)$ to $p_{k}^{\prime}$. In particular, the curve class $F_{k}-E_{k} \in \operatorname{Pic}(Y)$ is mapped to $E_{k}^{\prime}$.

We now need to check that the composition of isomorphisms $\left(D^{\prime}\right)^{\perp} \cong K \cong D^{\perp}$ given in Theorem 5.5 coincides with $\mu_{k}^{*}:\left(D^{\prime}\right)^{\perp} \rightarrow D^{\perp}$. To do so, suppose given $\sum a_{i} e_{i}=\sum a_{i}^{\prime} e_{i}^{\prime} \in K$. Then $a_{i}=a_{i}^{\prime}$ for $i \neq k$ and $a_{k}^{\prime}=-a_{k}+\sum_{i}\left[\epsilon_{i k}\right]_{+} a_{i}$ by (2.3). These determine classes $C \in \operatorname{Pic}(\bar{Y})$, $C^{\prime} \in \operatorname{Pic}\left(\bar{Y}^{\prime}\right)$ as in Theorem 5.5. It is enough to check that if $\pi_{1}: Y_{k} \rightarrow \bar{Y}, \pi_{1}^{\prime}: Y_{k}^{\prime} \rightarrow \bar{Y}^{\prime}$ are the blowups at $p_{k}, p_{k}^{\prime}$, respectively, then $\mu_{k}^{*}\left(\left(\pi_{1}^{\prime}\right)^{*}\left(C^{\prime}\right)-a_{k}^{\prime} E_{k}^{\prime}\right)=\pi_{1}^{*}(C)-a_{k} E_{k}$. Call these two divisors $C_{1}^{\prime}$ and $C_{1}$, respectively. Since the Picard group of $Y_{k}$ is easily seen to be generated by the proper transforms of the toric divisors of $Y_{k}$, and $\mu_{k}$ takes the boundary divisor of $Y_{k}$ corresponding to $\rho \in \bar{\Sigma}$ to the boundary divisor of $Y_{k}^{\prime}$ corresponding to $T_{k}(\rho) \in \bar{\Sigma}^{\prime}$, it is enough to check that $C_{1}^{\prime}$ and $C_{1}$ have the same intersection numbers with the boundary divisors of $Y_{k}$. It is clear that $C_{1}^{\prime}$ and $C_{1}$ have the same intersection numbers with all boundary divisors except possibly those corresponding to the rays $\pm \mathbb{R}_{\geqslant 0} w_{k}$. Call these two divisors $D_{k, \pm} \subseteq Y_{k}$. Then

$$
C_{1} \cdot D_{k,+}=\sum_{i: w_{i}=w_{k}, i \neq k} a_{i}=C_{1}^{\prime} \cdot D_{k,+}
$$

while

$$
C_{1} \cdot D_{k,-}=\sum_{i: w_{i}=-w_{k}, i \neq k} a_{i}=C_{1}^{\prime} \cdot D_{k,-}
$$

This proves the result. 


\section{Mark Gross, Paul Hacking and Sean Keel}

\section{Examples of non-finitely-generated upper cluster algebras}

We will now use the material of the previous two sections to construct examples of non-finitelygenerated upper cluster algebras with principal coefficients and with general coefficients. These examples are a generalization of the example of Speyer [Spe13]. They fail to be finitely generated as a consequence of the following result.

Lemma 6.1. Let $A$ be a ring, let $R$ be an $M=\mathbb{Z}^{n}$-graded $A$-algebra and let $R=\bigoplus_{m \in M} R_{m}$. If $R_{0}=A$ and $R_{m}$ is not a finitely generated $A$-module for some $m \in M$, then $R$ is not Noetherian.

Proof. Let $I$ be the homogeneous ideal of $R$ generated by $R_{m}$. We show that $I$ is not finitely generated as an ideal. Suppose to the contrary that homogeneous $f_{1}, \ldots, f_{p} \in R$ generate $I$. Necessarily $f_{i}=\sum r_{i j} f_{i j}$ for some $r_{i j} \in R$ and $f_{i j} \in R_{m}$, so we can assume that $I$ is generated by a finite number of $f_{i j} \in R_{m}$. But $R_{m}=I \cap R_{m}$ is the $R_{0}$-module generated by the $f_{i j}$ and $R_{0}=A$, contradicting the assumption that $R_{m}$ is not finitely generated as an $A$-module.

In what follows, suppose given fixed data and seed data satisfying the hypotheses of Proposition 5.4. This gives rise to the family $\lambda:(\mathcal{Y}, \mathcal{D}) \rightarrow T_{K^{*}}$ of Loojienga pairs, obtained by blowing up a sequence of centers $Z_{1}, \ldots, Z_{n} \subseteq \bar{Y} \times T_{K^{*}}$ in some order, for $\bar{Y}$ a toric surface.

Theorem 6.2. Let $(Y, D)$ be the general fibre of $(\mathcal{Y}, \mathcal{D}) \rightarrow T_{K^{*}}$. Suppose that every irreducible component of $D$ has self-intersection -2 . Then,

(i) the algebra $\Gamma\left(\mathcal{A}_{\text {prin }}, \mathcal{O}_{\mathcal{A}_{\text {prin }}}\right)$ is non-Noetherian;

(ii) for $t \in T_{M}$ very general and $\mathcal{A}_{t}$ the corresponding cluster variety with general coefficients, $\Gamma\left(\mathcal{A}_{t}, \mathcal{O}_{\mathcal{A}_{t}}\right)$ is non-Noetherian.

Proof. Let $X$ be, as usual, the subset of $\mathcal{X}$ obtained by gluing together the initial seed torus $\mathcal{X}_{\mathbf{s}}$ and adjacent seed tori $\mathcal{X}_{\mu_{k}(\mathbf{s})}$. By Corollary 4.6, $\Gamma\left(\mathcal{A}_{\text {prin }}, \mathcal{O}_{\mathcal{A}_{\text {prin }}}\right)=\bigoplus_{m \in M^{\circ}} \Gamma\left(X, \mathcal{L}_{m}\right)$, and this gives an $M^{\circ}$-grading on this algebra. In addition, by Lemma 5.2, $X$ and $\mathcal{Y} \backslash \mathcal{D}$ agree outside a set of codimension at least two, and both $X$ and $\mathcal{Y} \backslash \mathcal{D}$ are non-singular. So $\operatorname{Pic} X \cong \operatorname{Pic}(\mathcal{Y} \backslash \mathcal{D})$. Thus for each $m \in M^{\circ}, \mathcal{L}_{m}$ can be viewed as a line bundle on $\mathcal{Y} \backslash \mathcal{D}$, and $\mathcal{L}_{m}$ has the same space of sections regardless of whether $\mathcal{L}_{m}$ is viewed as a bundle on $X$ or on $\mathcal{Y} \backslash \mathcal{D}$. So, by the lemma, it will suffice to show that $\Gamma\left(\mathcal{Y} \backslash \mathcal{D}, \mathcal{O}_{\mathcal{Y}}\right)=A:=\mathbb{k}[K]$ and find some line bundle $\mathcal{L}$ on $\mathcal{Y} \backslash \mathcal{D}$ such that $\Gamma(\mathcal{Y} \backslash \mathcal{D}, \mathcal{L})$ is not a finitely generated $A$-module.

To show $\Gamma\left(\mathcal{Y} \backslash \mathcal{D}, \mathcal{O}_{\mathcal{Y}}\right)=A$, it is sufficient to show that a regular function on $\mathcal{Y} \backslash \mathcal{D}$ must be constant on the very general fibre of $\lambda: \mathcal{Y} \backslash \mathcal{D} \rightarrow T_{K^{*}}=\operatorname{Spec} \mathbb{k}[K]$. So consider the fibre $(Y, D)$ of $(\mathcal{Y}, \mathcal{D}) \rightarrow T_{K^{*}}$ over $\phi \in T_{K^{*}}$. The space of regular functions on $Y \backslash D$ can be identified with $\lim _{\rightarrow} H^{0}\left(Y, \mathcal{O}_{Y}(n D)\right)$. Consider the long exact cohomology sequence associated with

$$
\left.0 \rightarrow \mathcal{O}_{Y}(n D) \rightarrow \mathcal{O}_{Y}((n+1) D) \rightarrow \mathcal{O}_{Y}((n+1) D)\right|_{D} \rightarrow 0 .
$$

Note that $D \in D^{\perp}$ since all components of $D$ have square -2 . Also note that with $\phi \in T_{K^{*}}$ $=\operatorname{Hom}\left(D^{\perp}, \operatorname{Pic}^{0}(D)\right), \phi(D)$ is the normal bundle of $D$ in $Y$ by Theorem 5.5. Thus as $\phi$ is very general, $\phi(D)$ is not torsion. So $H^{0}\left(D, \mathcal{O}_{D}(n D)\right)=0$ for all $n>0$, and we see that $H^{0}\left(Y, \mathcal{O}_{Y}(n D)\right)=\mathbb{k}$ for all $n \geqslant 0$. Thus the only regular functions on $Y \backslash D$ are constant.

Now let $E$ be the exceptional divisor over the last center $Z_{i}$ blown up in constructing $\mathcal{Y}$, so that $E$ is a $\mathbb{P}^{1}$-bundle over $T_{K^{*}}$. Then we claim that $\Gamma\left(\mathcal{Y} \backslash \mathcal{D}, \mathcal{O}_{\mathcal{Y}}(E)\right)$ is not a finitely generated $A$-module. Note that

$$
\Gamma\left(\mathcal{Y} \backslash \mathcal{D}, \mathcal{O}_{\mathcal{Y}}(E)\right)=\lim _{n \geqslant 0} \Gamma\left(\mathcal{Y}, \mathcal{O}_{\mathcal{Y}}(E+n \mathcal{D})\right)
$$




\section{BiRATIONAL GEOMETRY OF CLUSTER ALGEBRAS}

Since each of these groups is an $A$-module, it is sufficient to show that the increasing chain of $A$-modules

$$
\Gamma\left(\mathcal{Y}, \mathcal{O}_{\mathcal{Y}}(E)\right) \subseteq \Gamma\left(\mathcal{Y}, \mathcal{O}_{\mathcal{Y}}(E+\mathcal{D})\right) \subseteq \Gamma\left(\mathcal{Y}, \mathcal{O}_{\mathcal{Y}}(E+2 \mathcal{D})\right) \subseteq \cdots
$$

does not stabilize. We have a long exact sequence

$$
\begin{aligned}
0 \longrightarrow H^{0}\left(\mathcal{Y}, \mathcal{O}_{\mathcal{Y}}(E+(n-1) \mathcal{D})\right) \stackrel{i}{\longrightarrow} H^{0}\left(\mathcal{Y}, \mathcal{O}_{\mathcal{Y}}(E+n \mathcal{D})\right) \longrightarrow H^{0}\left(\mathcal{D}, \mathcal{O}_{\mathcal{D}}(E+n \mathcal{D})\right) \\
\longrightarrow H^{1}\left(\mathcal{Y}, \mathcal{O}_{\mathcal{Y}}(E+(n-1) \mathcal{D})\right) \longrightarrow H^{1}\left(\mathcal{Y}, \mathcal{O}_{\mathcal{Y}}(E+n \mathcal{D})\right) \longrightarrow H^{1}\left(\mathcal{D}, \mathcal{O}_{\mathcal{D}}(E+n \mathcal{D})\right)
\end{aligned}
$$

If $(Y, D)$ is any fibre of $(\mathcal{Y}, \mathcal{D}) \rightarrow T_{K^{*}}$, one checks easily that $H^{1}\left(Y, \mathcal{O}_{Y}(E \cap Y)\right)=0$ (as $E \cap Y$ is an irreducible -1-curve) and that $H^{1}\left(D, \mathcal{O}_{D}((E \cap D)+n D)\right)=0$ (using that $E \cap D$ consists of one point). It then follows from cohomology and base change along with the fact that $T_{K^{*}}$ is affine that $H^{1}\left(\mathcal{D}, \mathcal{O}_{\mathcal{D}}(E+n \mathcal{D})\right)=0$ for all $n \geqslant 0$ and $H^{1}\left(\mathcal{Y}, \mathcal{O}_{\mathcal{Y}}(E)\right)=0$. Inductively from the long exact sequence above one sees $H^{1}\left(\mathcal{Y}, \mathcal{O}_{\mathcal{Y}}(E+n \mathcal{D})\right)=0$ for all $n \geqslant 0$. Thus the cokernel of the inclusion $i$ in the long exact sequence above is $H^{0}\left(\mathcal{D}, \mathcal{O}_{\mathcal{D}}(E+n \mathcal{D})\right)$. Now $\lambda_{*} \mathcal{O}_{\mathcal{D}}(E+n \mathcal{D})$ is a line bundle on $T_{K^{*}}$, again by cohomology and base change, and since $T_{K^{*}}$ is an algebraic torus, this line bundle must be trivial. Thus $H^{0}\left(\mathcal{D}, \mathcal{O}_{\mathcal{D}}(E+n \mathcal{D})\right)=A$, and we see that the chain (6.1) never stabilizes.

The argument for $\mathcal{A}_{t}$ for $t$ very general is identical but easier, as we have already done the relevant cohomology calculations on $(Y, D)$ a very general fibre of $(\mathcal{Y}, \mathcal{D}) \rightarrow T_{K^{*}}$. Then one makes use of Corollary 4.6, (3).

Example 6.3. Using Construction 5.3 it is easy to produce many examples satisfying the hypotheses of the theorem above. For example, let $\bar{\Sigma}$ be the fan for $\mathbb{P}^{2}$, with rays generated by $w_{1}=w_{2}=w_{3}=(1,0), w_{4}=w_{5}=w_{6}=(0,1)$ and $w_{7}=w_{8}=w_{9}=(-1,-1)$. Take all $\nu_{i}=1$. Thus a general $(Y, D)$ involves blowing three points on each of the coordinate lines of $\mathbb{P}^{2}$, so $D$ is a cycle of three $(-2)$-curves.

This is very closely related to the example of Speyer [Spe13], which in the terminology of construction 5.3 again involves taking the fan for $\mathbb{P}^{2}, w_{1}=(1,0), w_{2}=(0,1)$ and $w_{3}=(-1,-1)$, but taking all $\nu_{i}=3$. The surface $(Y, D)$ will be constructed by performing a weighted blowup of one point on each of three coordinate lines on $\mathbb{P}^{2}$. Then $D$ is still a cycle of three $(-2)$-curves, but the situation requires some additional analysis because $Y$ is in fact singular (having three $A_{2}$-singularities).

Remark 6.4. In fact, there is a much broader range of counterexamples: suppose that the blowup $(\mathcal{Y}, \mathcal{D}) \rightarrow \bar{Y} \times T_{K^{*}}$ factors through $(\mathcal{Y}, \mathcal{D}) \rightarrow\left(\mathcal{Y}^{\prime}, \mathcal{D}^{\prime}\right)$, such that a very general fibre $\left(Y^{\prime}, D^{\prime}\right)$ of $\left(\mathcal{Y}^{\prime}, \mathcal{D}^{\prime}\right) \rightarrow T_{K^{*}}$ has the property that every irreducible component of $D^{\prime}$ has self-intersection -2 . Then the argument above shows that the Cox ring of $Y^{\prime} \backslash D^{\prime}$ is non-Noetherian, and $Y^{\prime} \backslash D^{\prime}$ is an open subset of $Y \backslash D$. If $U \subseteq V$, then the Cox ring of $V$ surjects onto the Cox ring of $U$, so the fact that the Cox ring of $Y^{\prime} \backslash D^{\prime}$ is non-Noetherian implies that the Cox ring of $Y \backslash D$ is non-Noetherian. A similar but slightly more delicate argument also applies to the principal coefficient case.

In fact, we expect that whenever the intersection matrix of $D$ is negative definite, the Noetherian condition fails.

\section{Counterexamples to the Fock-Goncharov dual bases conjecture}

Fock and Goncharov [FG09] gave an explicit conjecture about the existence of $\mathbb{k}$-bases for the $\mathcal{X}$ and $\mathcal{A}$ cluster algebras. We will state it loosely here, under the assumption that all $d_{i}=1$, so 


\section{Mark Gross, Paul Hacking and Sean Keel}

that $M=M^{\circ}, N=N^{\circ}$. This merely allows us to avoid discussing Langlands dual seeds.

Conjecture 7.1 [FG09], 4.1. The group $N$ parameterizes a canonical basis for $H^{0}\left(\mathcal{X}, \mathcal{O}_{\mathcal{X}}\right)$, and $M$ parameterizes a canonical basis of sections for $H^{0}\left(\mathcal{A}, \mathcal{O}_{\mathcal{A}}\right)$.

In fact, the conjecture as stated in [FG09] is much stronger, giving an explicit conjectural description of the bases as the set of positive universal Laurent polynomials which are extremal, that is, not a non-trivial sum of two other positive universal Laurent polynomials. This strongest form of the conjecture has now been disproven in [LLZ14], in which examples are given where the set of all extremal positive universal Laurent polynomials are not linearly independent. Here we give a much more basic counterexample to a much weaker form of the conjecture.

We shall again restrict to the case where there are no frozen variables. We will merely assume that the conjectured basis is compatible with the $T_{K}$ action on $\mathcal{A}$ given by Remark 2.2(iii), and the map $\lambda: \mathcal{X} \rightarrow T_{K^{*}}$ in the natural way. We assume that the canonical basis element of $\Gamma\left(\mathcal{X}, \mathcal{O}_{\mathcal{X}}\right)$ corresponding to $n \in K$ is $\lambda^{*}\left(z^{n}\right)$. Furthermore, for $\pi: M \rightarrow K^{*}$ the natural projection dual to the inclusion $K \rightarrow N$, we assume that the set $\pi^{-1}(m)$ parameterizes a basis for the subspace of $H^{0}\left(\mathcal{A}, \mathcal{O}_{\mathcal{A}}\right)$ of weight $m$ eigenvectors for the $T_{K}$ action.

We indicate now why a basis with these properties cannot exist in general. We consider the rank two cluster algebras produced by construction 5.3, following the notation of the construction, taking all $\nu_{i}=1$. The general fibre of $\lambda: \mathcal{X} \rightarrow T_{K^{*}}$ is isomorphic outside codimension two subsets to the general fibre of $\lambda: \mathcal{Y} \backslash \mathcal{D} \rightarrow T_{K^{*}}$. A fibre of the latter map is of the form $U:=Y \backslash D$, where $(Y, D)$ is a Looijenga pair with a map $Y \rightarrow \bar{Y}$ obtained by blowing up points on the toric boundary. Since the initial data of the $w_{i}$ in construction 5.3 can be chosen arbitrarily, and, in particular the points $w_{i}$ may be repeated as many times as we like, we can easily find examples for which $D \subseteq Y$ is analytically contractible, that is, for which there is an analytic map $(Y, D) \rightarrow\left(Y^{\prime}, p\right)$ with exceptional locus $D$ and $p \in Y^{\prime}$ a single point. Further, $U=Y \backslash D=Y^{\prime} \backslash\{p\}$, and so $H^{0}\left(U, \mathcal{O}_{U}\right)=H^{0}\left(Y^{\prime}, \mathcal{O}_{Y^{\prime}}\right)=\mathbb{k}$. Even if $D$ is not contractible but rather a cycle of $(-2)$-curves as in Theorem 6.2, the very general fibre $Y \backslash D$ will only have constant functions. It follows that

$$
H^{0}\left(\mathcal{X}, \mathcal{O}_{\mathcal{X}}\right)=H^{0}\left(T_{K^{*}}, \mathcal{O}_{T_{K^{*}}}\right)=\mathbb{k}[K] .
$$

Thus there are no functions for points of $N \backslash K$ to parameterize.

Consider the conjecture in the opposite direction. Assume for simplicity that, as in Corollary 4.7, $\operatorname{Pic}(X)=\operatorname{Pic}\left(X_{t}\right)=M / p^{*}(N)$ is torsion free. Then $\left(M / p^{*}(N)\right)^{*}=K=\operatorname{Ker}\left(p^{*}: N \rightarrow M\right)$. The Fock-Goncharov conjecture for $\mathcal{A}_{\text {prin }}$ implies the analogous result for very general $\mathcal{A}_{t}$, that is, the existence of a canonical basis of the upper cluster algebra with very general coefficients $H^{0}\left(\mathcal{A}_{t}, \mathcal{O}\right)$, parameterized by $\mathcal{X}^{\text {trop }}(\mathbb{Z})$. We have

$$
H^{0}\left(\mathcal{A}_{t}, \mathcal{O}_{\mathcal{A}_{t}}\right)=\operatorname{Cox}\left(X_{i(t)}\right)=\bigoplus_{m \in K^{*}=\operatorname{Pic}\left(X_{i(t)}\right)} H^{0}\left(X_{i(t)}, \mathcal{L}_{m}\right)
$$

by Corollary 4.6. Here $\mathcal{L}_{m}$ is a line bundle representing the isomorphism class given by $m$. Assuming that the canonical bases are compatible with the natural torus actions, for $m \in K^{*}$, $\pi^{-1}(m) \subset \mathcal{X}^{\operatorname{trop}}(\mathbb{Z})$ restricts to a basis for the weight $m$-eigenspace $H^{0}\left(X_{i(t)}, \mathcal{L}_{m}\right)$ of $H^{0}\left(\mathcal{A}_{t}, \mathcal{O}\right)$ under the $T_{K}$ action, for $\pi: \mathcal{X}^{\operatorname{trop}}(\mathbb{Z}) \rightarrow K^{*}=M / p^{*}(N)$, the natural map induced by the fibration $\mathcal{X} \rightarrow T_{K^{*}}$. But any choice of seed identifies $\mathcal{X}^{\operatorname{trop}}(\mathbb{Z})$ with $M$ and each fibre of $\pi$ with a $p^{*}(N)=N / K$ torsor. Thus the conjecture implies that all line bundles on $X_{t}$ have isomorphic spaces of sections, with basis parameterized (after choice of seed) by an $N / K$-affine space. This 


\section{BiRATIONAL GEOMETRY OF CLUSTER ALGEBRAS}

is a very strong condition - most varieties have line bundles with no non-trivial sections, and rather than an affine space one would expect (for example by comparison with the toric case) sections parameterized by integer points of a polytope. Explicitly, the example of Theorem 6.2 clearly has line bundles with non-isomorphic spaces of sections.

This reasoning suggests to us that the conjecture can only hold if $\mathcal{X}$ is affine up to flops.

CONJECTURE 7.2. If the Fock-Goncharov conjecture holds then $H^{0}\left(\mathcal{X}, \mathcal{O}_{\mathcal{X}}\right)$ is finitely generated, and the canonical map $\mathcal{X} \rightarrow \operatorname{Spec}\left(H^{0}\left(\mathcal{X}, \mathcal{O}_{\mathcal{X}}\right)\right)$ is an isomorphism, outside a codimension two set.

The results of $\S 5$ imply that when the hypotheses of Theorem 5.6 are satisfied, the conditions in Conjecture 7.2 hold if and only if the generic fibre of $\mathcal{X} \rightarrow T_{K^{*}}$ is affine, which is true if and only if the canonical symmetric form on $K$ given by Theorem 5.6 is negative definite. Indeed, the generic fibre of $\mathcal{X} \rightarrow T_{K^{*}}$ is isomorphic, outside a codimension two set, to a surface $Y \backslash D$ as in Theorem 5.5. But if $Y \backslash D$ is affine, then $D$ supports an ample divisor, and by the Hodge index theorem, $D^{\perp}$ is negative definite. Conversely, if $D^{\perp}$ is negative definite, there must be some integers $a_{i}$ such that $\left(\sum a_{i} D_{i}\right)^{2}>0$. The result then follows from [GHK11, Lemma 6.8] and the fact that $(Y, D)$ is chosen generally in the family.

\section{ACKNOWLEDGEMENTS}

The genesis of our results on cluster varieties was a conversation with M. Kontsevich. He pointed out to us that (in the skew-symmetric case) a seed is the same thing as a collection of vectors in a symplectic lattice and the piecewise linear cluster mutation is just like moving worms in the integral affine manifolds central to mirror symmetry for open Calabi-Yau varieties; see, for example, [GHK11]. Before this conversation we had been incorrectly assuming the cluster picture was a very special case of the mirror construction in [GHK11]. However, Kontsevich's remarks led us to the correct view that in dimension two, the scope of the two theories is exactly the same. This in turn led to the simple blowup description of cluster varieties we describe here.

We first learned of the connection between mirror symmetry and cluster varieties from conversations with A. Neitzke. We received considerable inspiration from conversations with V. Fock, S. Fomin, A. Goncharov, B. Keller, B. Leclerc, G. Musiker, M. Shapiro, Y. Soibelman, and D. Speyer. Special thanks go to Greg Muller, who pointed out a crucial mistake in a draft version of this paper; see Remark 3.13.

\section{REFERENCES}

Arz09 I. Arzhantsev, On the factoriality of Cox rings (Russian), Mat. Zametki 85 (2009), no. 5, 643651; translation in Math. Notes 85 (2009), no. 5-6, 623-629. http://dx.doi.org/10.1134/ S0001434609050022

BCHM10 C. Birkar, P. Cascini, C. Hacon and J. McKernan, Existence of minimal models for varieties of log general type, J. Amer. Math. Soc. 23 (2010), no. 2, 405-468. http://dx.doi.org/10. 1090/S0894-0347-09-00649-3

BFZ05 A. Berenstein, S. Fomin and A. Zelevinsky, Cluster algebras III: Upper bounds and double Bruhat cells, Duke Math. J. 126 (2005), 1-52. http://dx.doi.org/10.1215/ S0012-7094-04-12611-9

BH03 F. Berchtold, J. Hausen, Homogeneous coordinates for algebraic varieties, J. Algebra 266 (2003), 636-670. http://dx.doi.org/10.1016/S0021-8693(03)00285-0 


\section{Mark Gross, Paul Hacking and Sean Keel}

Dyn52 E. Dynkin, Semisimple subalgebras of semisimple Lie algebras, Mat. Sb. Nov. Ser. 3 (1952) 349-462.

FG06 V. Fock and A. Goncharov, Moduli spaces of local systems and higher Teichmüller theory, Publ. Math. Inst. Hautes Études Sci. 103 (2006), 1-211. http://dx.doi.org/10.1007/ s10240-006-0039-4

FG09 V. Fock and A. Goncharov, Cluster ensembles, quantization and the dilogarithm, Ann. Sci. Éc. Norm. Supér. (4) 42 (2009), no. 6, 865-930.

FG11 V. Fock and A. Goncharov, Cluster X-varieties at infinity, arXiv:1104.0407.

FZ02a S. Fomin and A. Zelevinsky, Cluster algebras I: Foundations, J. Amer. Math. Soc. 15 (2002), 497-529. http://dx.doi.org/10.1090/S0894-0347-01-00385-X

FZ02b S. Fomin and A. Zelevinsky, The Laurent phenomenon, Adv. in Appl. Math. 28 (2002), 119144. http://dx.doi.org/10.1006/aama.2001.0770

FZ07 S. Fomin and A. Zelevinsky, Cluster algebras IV: Coefficients, Compos. Math. 143 (2007), 112-164. http://dx.doi.org/10.1112/S0010437X06002521

GHK11 M. Gross, P. Hacking and S. Keel, Mirror symmetry for log Calabi-Yau surfaces I, Inst. Hautes Études Sci. Publ. Math. (to appear), arXiv:1106.4977.

GHK15 M. Gross, P. Hacking and S. Keel, Moduli of surfaces with an anti-canonical cycle, Compos. Math. 151 (2015), no. 2, 265-291. http://dx.doi.org/10.1112/S0010437X14007611

GHKK14 M. Gross, P. Hacking, S. Keel and M. Kontsevich, Canonical bases for cluster algebras, arXiv: 1411.1394.

Gro60 A. Grothendieck, Éléments de géométrie algébrique I, Le langage des schémas, Publ. Math. Inst. Hautes Études Sci. 4 (1960).

GS11 M. Gross and B. Siebert, From real affine geometry to complex geometry, Ann. of Math. 174 (2011), 1301-1428. http://dx.doi.org/10.4007/annals.2011.174.3.1

Har77 R. Hartshorne, Algebraic Geometry, Graduate Texts in Mathematics, Vol. 52, Springer-Verlag, New York-Heidelberg, 1977.

HK00 Y. Hu and S. Keel, Mori dream spaces and GIT, Dedicated to William Fulton on the occasion of his 60th birthday, Michigan Math. J. 48 (2000), 331-348. http://dx.doi.org/10.1307/ $\mathrm{mmj} / 1030132722$

HKT09 P. Hacking, S. Keel, and J. Tevelev, Stable pair, tropical, and log canonical compactifications of moduli spaces of del Pezzo surfaces, Invent. Math. 178 (2009), no. 1, 173-227. http://dx. doi.org/10.1007/s00222-009-0199-1

Iit77 S. Iitaka, On logarithmic Kodaira dimension of algebraic varieties in Complex analysis and algebraic geometry, 175-189, Iwanami Shoten, Tokyo, 1977.

Kel12 B. Keller, Cluster algebras and derived categories in Derived categories in algebraic geometry, 123-183, EMS Ser. Congr. Rep., Eur. Math. Soc., Zürich, 2012. http://dx. doi .org/10.4171/ $115-1 / 6$

KM98 J. Kollár and S. Mori, Birational geometry of algebraic varieties, Cambridge Tracts in Math. 134, Cambridge University Press, 1998. http://dx.doi.org/10.1017/ CB09780511662560

KS06 M. Kontsevich and Y. Soibelman, Affine structures and non-Archimedean analytic spaces in The unity of mathematics (P. Etingof, V. Retakh, I.M. Singer, eds.), 321-385, Progr. Math. 244, Birkhäuser, 2006. http://dx.doi.org/10.1007/0-8176-4467-9_9

LLZ14 K. Lee, L. Li and A. Zelevinsky, Positivity and tameness in rank 2 cluster algebras, J. Algebraic Combin. 40 (2014), no. 3, 823-840. http://dx.doi.org/10.1007/s10801-014-0509-6

Loo81 E. Looijenga, Rational surfaces with an anticanonical cycle, Ann. of Math. (2) 114 (1981), no. 2, 267-322.

LP12 T. Lam and P. Pylyavskyy, Laurent phenomenon algebras, arXiv:1206.2611 


\section{BiRATIONAL GEOMETRY OF CLUSTER ALGEBRAS}

Lus90 G. Lusztig, Canonical bases arising from quantized enveloping algebras, J. Amer. Math. Soc. 3 (1990), no. 2, 447-498. http://dx.doi.org/10.1090/S0894-0347-1990-1035415-6

Per90 U. Persson, Configurations of Kodaira fibers on rational elliptic surfaces, Math. Z. 205 (1990), 1-47. http://dx.doi.org/10.1007/BF02571223

Spe13 D. Speyer, An infinitely generated upper cluster algebra, arXiv:1305.6867.

Mark Gross mgross@dpmms.cam.ac.uk

DPMMS, Centre for Mathematical Sciences, Wilberforce Road, Cambridge, CB3 0WB, United Kingdom

Paul Hacking hacking@math.umass.edu

Department of Mathematics and Statistics, Lederle Graduate Research Tower, University of Massachusetts, Amherst, MA 01003-9305, USA

Sean Keel keel@math.utexas.edu

Department of Mathematics, 1 University Station C1200, Austin, TX 78712-0257, USA 